\title{
FILTERED SPECTRA ARISING FROM PERMUTATIVE CATEGORIES
}

\author{
GREGORY ARONE AND KATHRYN LESH
}

\begin{abstract}
Given a special $\Gamma$-category $\mathcal{C}$ satisfying some mild hypotheses, we construct a sequence of spectra interpolating between the spectrum associated to $\mathcal{C}$ and the Eilenberg-Mac Lane spectrum $\mathrm{H} \mathbb{Z}$. Examples of categories to which our construction applies are: the category of finite sets, the category of finite-dimensional vector spaces, and the category of finitely-generated free modules over a reasonable ring. In the case of finite sets, our construction recovers the filtration of $\mathrm{H} \mathbb{Z}$ by symmetric powers of the sphere spectrum. In the case of finite-dimensional complex vector spaces, we obtain an apparently new sequence of spectra, $\left\{A_{m}\right\}$, that interpolate between $b u$ and $\mathrm{H} \mathbb{Z}$. We think of $A_{m}$ as a "bu-analogue" of $S p^{m}(\mathbf{S})$ and describe far-reaching formal similarities between the two sequences of spectra. For instance, in both cases the $m$ th subquotient is contractible unless $m$ is a power of a prime, and in $v_{k}$-periodic homotopy the filtration has only $k+2$ nontrivial terms. There is an intriguing relationship between the $b u$-analogues of symmetric powers and Weiss's orthogonal calculus, parallel to the not yet completely understood relationship between the symmetric powers of spheres and the Goodwillie calculus of homotopy functors. We conjecture that the sequence $\left\{A_{m}\right\}$, when rewritten in a suitable chain complex form, gives rise to a minimal projective resolution of the connected cover of bu. This conjecture is the bu-analogue of a theorem of Kuhn and Priddy about the symmetric power filtration. The calculus of functors provides substantial supporting evidence for the conjecture.
\end{abstract}

\section{INTRODUCTION}

In this paper we define and investigate a filtration on spectra that arise from the application of Segal's infinite loop space machine to certain permutative categories. The input to our construction is an "augmented" permutative category $\mathcal{C}$ (Definition 2.1), and the output is a sequence of spectra interpolating between the spectrum associated to $\mathcal{C}$ and the Eilenberg-Mac Lane spectrum $\mathrm{H} \mathbb{Z}$. Examples of categories to which the construction applies are: the category of finite sets, the category of finite-dimensional vector spaces over a field, the category of finitelygenerated free modules over a ring satisfying the dimension invariance property, and the category of finitely-generated free groups. The motivating example is the category of finite sets, to which Segal's infinite loop space machine associates the sphere spectrum, S. For this example, the construction turns out to give the symmetric power filtration,

$$
\mathbf{S}=\mathrm{Sp}^{1}(\mathbf{S}) \rightarrow \mathrm{Sp}^{2}(\mathbf{S}) \rightarrow \cdots \rightarrow \mathrm{Sp}^{\infty}(\mathbf{S}) \simeq \mathrm{H} \mathbb{Z},
$$

Date: December 24, 2005.

1991 Mathematics Subject Classification. Primary 55P47; Secondary 55P42, 55P48.

The authors were partially supported by the National Science Foundation, Grant DMS0307069 . 
which interpolates between the sphere spectrum $\mathbf{S}$ and the infinite symmetric power $\mathrm{Sp}^{\infty}(\mathbf{S}) \simeq \mathrm{H} \mathbb{Z}$. The example of finite sets was studied extensively by the second author in [11, 12], and this paper is, among other things, a generalization of that work. In effect, we show that Lesh's model for the symmetric power filtration can be generalized by lifting the group-theoretic constructions of [12] to a categorical level.

Throughout the paper, we work with a (possibly topological) permutative category $\mathcal{C}$ that happens to be a disjoint union of small categories, $\mathcal{C}=\coprod_{n} \mathcal{C}_{n}$. We call $\mathcal{C}_{n}$ the " $n$th component" of $\mathcal{C}$. Let $A$ be the spectrum associated to $\mathcal{C}$.

The first part of the paper gives a general categorical construction of a sequence of spectra that interpolate between $A$ and $\mathrm{H} \mathbb{Z}$. In Construction 3.8, we define inductively a sequence of permutative categories

$$
\mathcal{C}=\mathcal{K}_{0} \mathcal{C} \rightarrow \mathcal{K}_{1} \mathcal{C} \rightarrow \cdots \rightarrow \mathcal{K}_{\infty} \mathcal{C} \simeq \mathbb{Z}_{\geq 0}
$$

each of which, like $\mathcal{C}$ itself, is a disjoint union of components indexed by nonnegative integers. The category $\mathcal{K}_{m} \mathcal{C}$ is obtained from $\mathcal{K}_{m-1} \mathcal{C}$ by using a suitable homotopy pushout in the category of permutative categories to "kill" the $m$ th component of $\mathcal{K}_{m-1} \mathcal{C}$, which is denoted $\mathcal{K}_{m-1} \mathcal{C}_{m}$ and is the bottom nontrivial component. The category $\mathcal{K}_{\infty} \mathcal{C}$ is defined as the colimit, and the nerve of each component of $\mathcal{K}_{\infty} \mathcal{C}$ is contractible, so $\left|\mathcal{K}_{\infty} \mathcal{C}\right| \simeq \mathbb{Z}_{\geq 0}$.

After applying Segal's infinite loop space machine to the sequence (1.1), we obtain a sequence of spectra

$$
A=A_{0} \rightarrow A_{1} \rightarrow \cdots \rightarrow A_{\infty} \simeq \mathrm{H} \mathbb{Z},
$$

where $A_{m}$ is the spectrum associated to $\mathcal{K}_{m} \mathcal{C}$. We observe in Theorem 3.9 that the inductive definition of $\mathcal{K}_{m} \mathcal{C}$ leads to the expression of $A_{m}$ as a homotopy pushout:

$$
A_{m} \simeq \operatorname{hocolim}\left(\begin{array}{c}
\Sigma^{\infty}\left(B \mathcal{K}_{m-1} \mathcal{C}_{m}\right)_{+} \longrightarrow A_{m-1} \\
\downarrow \\
\mathbf{S}
\end{array}\right) .
$$

Thus it follows that we have a formula for the subquotients,

$$
A_{m} / A_{m-1} \simeq \Sigma^{\infty} \Sigma\left(B \mathcal{K}_{m-1} \mathcal{C}_{m}\right) \text {. }
$$

In particular, it is a basic property of our construction that the subquotients $A_{m} / A_{m-1}$ are always suspension spectra.

In the middle part of the paper, which is the most technical portion, we consider the spaces $B \mathcal{K}_{m-1} \mathcal{C}_{m}$ that appear in (1.3). Because of the inductive definition of the categories $\mathcal{K}_{m} \mathcal{C}$, the classifying space $B \mathcal{K}_{m-1} \mathcal{C}_{m}$ is not easy to analyze in general. However, by making two sets of simplifying assumptions, we can identify these spaces in more concrete terms.

The first simplification allows us to construct models for the categories $\mathcal{K}_{m} \mathcal{C}$ that are not inductively defined, but rather are obtained by filtering a single large combinatorial construction. We consider categories $\mathcal{C}$ that have an underlying "monogenic" category $\mathcal{B}$, meaning that $\mathcal{B}$ is generated under coproducts by a single object $g$, the generator (Definition 4.1 ). We assume that $\mathcal{C}$ is a suitable category of "admissible" isomorphisms of $\mathcal{B}$ (Definition 4.2 ), and we also call $\mathcal{C}$ "monogenic." The categories of finite sets, finite-dimensional vector spaces, finitely-generated free modules and finitely-generated free groups are all monogenic. We also assume that the underlying category $\mathcal{B}$ satisfies an axiom that we call the Injectivity 
Axiom (Axiom 5.1). Roughly speaking, the axiom says that the canonical map from the coproduct to the product is injective. The categories of finite sets, finitedimensional vector spaces, and finitely-generated free modules satisfy the Injectivity Axiom, but the category of free groups does not. The assumption of an underlying monogenic category that satisfies the Injectivity Axiom allows us to introduce a combinatorially-defined permutative category $\mathcal{F}_{\infty} \mathcal{C}$ with a filtration by permutative categories $\mathcal{F}_{m} \mathcal{C}$ (Definition 4.5). These categories model the permutative categories $\mathcal{K}_{m} \mathcal{C}$ in the sense that $\mathcal{F}_{m} \mathcal{C}$ and $\mathcal{K}_{m} \mathcal{C}$ are related by a chain of monoidal functors each of which induces an equivalence of classifying spaces (Theorem 7.1).

The second simplification uses further assumptions on $\mathcal{C}$ to show that the category $\mathcal{F}_{m} \mathcal{C}$, which models $\mathcal{K}_{m} \mathcal{C}$, can be described as a union of orbit categories, generalizing the description in [12]. More concretely, if $g$ denotes the generator of the monogenic category $\mathcal{C}$ and $g^{n}$ denotes its $n$-fold coproduct with itself, we consider the groups $G_{n}=\operatorname{Aut}_{\mathcal{C}}\left(g^{n}\right)$. We define a collection of "standard" subgroups $\mathcal{H}\left(G_{n}\right)$ (Definition 8.1), which correspond in an appropriate sense to partial partitions of $g^{n}$. We filter this collection by collections $\mathcal{H}_{m}\left(G_{n}\right)$, which correspond to partitions whose "blocks" have size no larger than $m$. In the symmetric group case, the filtration corresponds to orbit length and was called the "complexity filtration" in $[12]$.

The following theorem is the main technical result of the middle part of the paper; it expresses the $\Gamma$-space $B \mathcal{K}_{m} \mathcal{C}$ in terms of the classifying spaces of the collections $\mathcal{H}_{m}\left(G_{n}\right)$. (See Section 8.)

Theorem 8.2. Let $\mathcal{C}$ be a monogenic category satisfying Axioms 5.1, 8.5, and 8.7. Then $B \mathcal{K}_{m} \mathcal{C}$ is monoidally equivalent to $\coprod_{n} B \mathcal{H}_{m}\left(G_{n}\right)$.

The conclusion of the theorem holds for the category of finite sets (with minor fiddling - see Corollary 8.4 and its proof), the category of finite-dimensional vector spaces, and the category of finitely-generated free modules over an integral domain in which $2 \neq 0$. On the other hand, the category of free groups does not satisfy any of our additional axioms. Thus, Construction 3.8 produces a filtration of the " $K$-theory of free groups" spectrum as in (1.2), but Theorem 8.2 does not apply.

Combining Theorem 8.2 with (1.3), we obtain the following corollary. In the case of finite sets, it essentially recovers [12], Theorem 1.2.

Corollary 8.3. Given a category $\mathcal{C}$ as in Theorem 8.2, let $\mathcal{R}_{m}$ be the collection $\mathcal{H}_{m-1}\left(G_{m}\right)$, and let $\epsilon: \Sigma^{\infty}\left(B \mathcal{R}_{m}\right)_{+} \rightarrow \mathbf{S}$ be induced by the map $B \mathcal{R}_{m} \rightarrow *$. Then

$$
A_{m} \simeq \operatorname{hocolim}\left(\begin{array}{c}
\Sigma^{\infty}\left(B \mathcal{R}_{m}\right)_{+} \longrightarrow A_{m-1} \\
\epsilon \downarrow \\
\mathbf{S}
\end{array}\right) .
$$

Corollary 8.3 allows us to show that in the example of finite sets, we obtain the same filtration as that studied by the second author in [12], and we recover the filtration from $S^{0}$ to $\mathrm{H} \mathbb{Z}$ by symmetric powers of the sphere spectrum.

Corollary 8.4. Let $A_{0} \rightarrow A_{1} \rightarrow \ldots$ be the sequence of spectra resulting from the application of Construction 3.8 to the category of finite sets and isomorphisms. Then $A_{m} \simeq \operatorname{Sp}^{m}(\mathbf{S})$.

The middle part of the paper is indispensable for the applications, but otherwise is probably rather tedious. One reason for this is that we worked quite hard to 
achieve sufficient generality to include the case of algebraic $K$-theory in Theorem 8.2 and Corollary 8.3, though we do not establish any further results about it at present. For now we confine ourselves to the remark that our filtration is reminiscent of, but not identical to, the rank filtration studied by J. Rognes in [17].

The remainder of the paper is devoted to a detailed study of the example of finitedimensional complex vector spaces and unitary isomorphisms, which gives rise to a filtration of the connective complex $K$-theory spectrum, $b u$. Our construction gives a sequence of spectra - new, as far as we can tell-interpolating between bu and $\mathrm{H} \mathbb{Z}$ :

$$
\text { bu }=A_{0} \rightarrow A_{1} \rightarrow \cdots \rightarrow A_{\infty} \simeq \mathrm{H} \mathbb{Z} .
$$

From here to the end of the introduction, we use $A_{m}$ to refer specifically to the $m$ th spectrum in (1.4), the unitary case. In view of Corollary 8.4, we think of $A_{m}$ as a "bu-analogue" of $\mathrm{Sp}^{m}(\mathbf{S})$, terminology that will be further justified below.

Perhaps the most interesting point made in this paper is that the sequence $\left\{A_{m}\right\}$ has a striking similarity to the sequence $\left\{\operatorname{Sp}^{m}(\mathbf{S})\right\}$, a similarity that goes considerably beyond the formal consequences of the general construction. The following facts about symmetric powers of spheres are the most relevant in describing the similarity. The notation $X^{\diamond}$ refers to the unreduced suspension of a space $X$. The notation $X_{\tilde{h} G}$ denotes the based homotopy orbits of a based $G$-action on $X$, i.e., $X_{\tilde{h} G}=\left(E G \times_{G} X\right) /\left(E G \times_{G} *\right)$.

- There is an equivalence of spectra

$$
\mathrm{Sp}^{m}(\mathbf{S}) / \operatorname{Sp}^{m-1}(\mathbf{S}) \simeq \Sigma^{\infty}\left(\left|\mathcal{P}_{m}\right|^{\diamond} \wedge S^{m}\right)_{\tilde{h} \Sigma_{m}},
$$

where $\mathcal{P}_{m}$ is the category of proper, nontrivial partitions of the set $\underline{m}$. ([2], Theorem 1.14)

- Let $p$ be a prime. The successive quotients $\operatorname{Sp}^{m}(\mathbf{S}) / \mathrm{Sp}^{m-1}(\mathbf{S})$ are trivial at $p$ except when $m=p^{k}$ for some $k$, and rationally trivial for $m \geq 2$. (This goes back at least to Nakaoka in the fifties, but also follows from [2], Theorems 8 and 1.14.)

- Let $p$ be a prime, $k>0$ an integer, and let $L(k)$ be the $p$-completion of $\Sigma^{-k} \mathrm{Sp}^{p^{k}}(\mathbf{S}) / \mathrm{Sp}^{p^{k-1}}(\mathbf{S})$. Then $L(k)$ is a wedge summand of a suspension spectrum. ([14])

- If $K(n)$ denotes the $n$th Morava $K$-theory, then

$$
K(n)_{*} \mathrm{Sp}^{p^{k}}(\mathbf{S}) / \mathrm{Sp}^{p^{k-1}}(\mathbf{S})=0
$$

for $n<k$. Moreover, the $p$-completion of the sequence

$$
\mathbf{S}=\mathrm{Sp}^{1}(\mathbf{S}) \rightarrow \mathrm{Sp}^{p}(\mathbf{S}) \rightarrow \mathrm{Sp}^{p^{2}}(\mathbf{S}) \rightarrow \cdots \rightarrow \mathrm{Sp}^{\infty}(\mathbf{S}) \simeq \mathrm{H} \mathbb{Z}
$$

terminates at $\mathrm{Sp}^{p^{k}}(\mathbf{S})$ in $v_{k}$-periodic homotopy. (An explicit reference for this statement is hard to find, but it is folklore knowledge among experts.)

The following four theorems summarize some of our main results about the filtration (1.4) of $b u$, each analogous to one of the points above about the symmetric powers of spheres.

Theorem 9.5. There is an equivalence of spectra

$$
A_{m} / A_{m-1} \simeq \Sigma^{\infty} \Sigma\left(\left|\mathcal{L}_{m}\right|^{\diamond} \wedge S^{2 m}\right)_{\tilde{h} U(m)},
$$

where $\mathcal{L}_{m}$ is the category of proper direct-sum decompositions of $\mathbb{C}^{m}$. 
Theorem 9.7. Let $p$ be a prime. The successive quotients $A_{m} / A_{m-1}$ are trivial at $p$ except when $m=p^{k}$ for some $k \geq 1$, and are rationally trivial for $m \geq 2$.

Because of Theorem 9.7 and its analogue for symmetric powers, we will often work at a prime $p$ and study $\operatorname{Sp}^{p^{k}}(\mathbf{S}) / \mathrm{Sp}^{p^{k-1}}(\mathbf{S})$ and $A_{p^{k}} / A_{p^{k-1}}$. We let $T(k)$ denote the $p$-completion of $\Sigma^{-(k+1)} A_{p^{k}} / A_{p^{k-1}}$, which turns out to be the $b u$-analogue of $L(k)$, and we write $\Gamma_{k}$ for an irreducible subgroup of the unitary group $U\left(p^{k}\right)$ that is described in Section 10.

Theorem 11.1. The spectrum $T(k)$ is a wedge summand of the suspension spectrum $\Sigma^{\infty}\left(S^{2 p^{k}}\right)_{\tilde{h} \Gamma_{k}}$.

Theorem 11.3. Let $p$ be a prime and let $K(n)$ denote the $n$th Morava $K$-theory. Then $K(n)_{*}\left(A_{p^{k}} / A_{p^{k-1}}\right)=0$ for $n<k$, and the sequence bu $=A_{0} \rightarrow A_{1} \rightarrow \mathcal{A}_{p} \rightarrow$ $A_{p^{2}} \rightarrow \cdots \rightarrow A_{\infty} \simeq \mathrm{H} \mathbb{Z}$ terminates at $A_{p^{k}}$ in $v_{k}$-periodic homotopy.

To further pursue the analogies between the symmetric powers of spheres and the spectra $A_{m}$, we need to bring the Goodwillie-Weiss calculus of functors into the picture. It turns out that just as the symmetric power filtration is related to the Goodwillie tower of the identity, so the $b u$-analogue is related to the Weiss tower of the functor $V \mapsto \mathrm{BU}(V)$.

We recall that it was shown in [3] that the Goodwillie tower of the identity functor, evaluated at $S^{1}$ and completed at a prime $p$, has the layers

$$
D_{p^{k}} \operatorname{Id}\left(S^{1}\right)=\Omega^{\infty} \operatorname{map}\left(S^{1} \wedge\left|\mathcal{P}_{p^{k}}\right|^{\triangleright}, \Sigma^{\infty} S^{p^{k}}\right)_{\tilde{h} \Sigma_{p^{k}}}
$$

and the tower converges to $S^{1}$ up to $p$-completion. Similarly, the Weiss tower for $V \mapsto \mathrm{BU}(V)$, evaluated at $V=\mathbb{C}$ and completed at $p$, has the layers

$$
D_{p^{k}} \mathrm{BU}(\mathbb{C})=\Omega^{\infty} \operatorname{map}\left(\left|\mathcal{L}_{p^{k}}\right|^{\diamond}, \Sigma^{\infty} S^{\mathrm{ad}_{p^{k}}} \wedge S^{2 p^{k}}\right)_{\tilde{h} U\left(p^{k}\right)}
$$

and the tower converges to $B U(1)$ up to $p$-completion. To shorten notation, we set

$$
\begin{aligned}
\mathrm{DI}_{k} & =D_{p^{k}} \operatorname{Id}\left(S^{1}\right) \\
\mathrm{DU}_{k} & =D_{p^{k}} \mathrm{BU}(\mathbb{C}) .
\end{aligned}
$$

It turns out that the infinite loop spaces of the subquotients of our filtration actually appear (up to a shift) as the layers in the Goodwillie and Weiss towers, as described in Theorem 11.2.

Theorem 11.2. For all $k \geq 0$, there are homotopy equivalences

$$
\begin{aligned}
B^{k-1} \mathrm{DI}_{k} & \simeq \Omega^{\infty} L(k) \\
B^{k-1} \mathrm{DU}_{k} & \simeq \Omega^{\infty} T(k) .
\end{aligned}
$$

The first part of the theorem is not new. It is proved in [2] using equations (1.5), (1.6), and a self-duality result for $\left|\mathcal{P}_{p^{k}}\right|^{\diamond}$. The proof of the second part of the theorem follows a similar plan, depending on Theorem 9.5, equation (1.7), and a self-duality result for $\left|\mathcal{L}_{p^{k}}\right|^{\diamond}$ (Corollary 10.2). The self-duality results for $\left|\mathcal{P}_{p^{k}}\right|^{\diamond}$ and $\left|\mathcal{L}_{p^{k}}\right|^{\diamond}$ follow in turn from the relationships of these complexes to Tits buildings for the general linear group and the symplectic group, respectively.

We close the paper with a series of conjectures that are based on the interaction of our construction and the calculus of functors. In the example of finite sets, the 
conjectures relate the Goodwillie tower of the identity functor, the symmetric powers of the sphere spectrum, and the Whitehead conjecture (now a theorem of Kuhn, and Kuhn and Priddy). In the example of finite-dimensional complex vector spaces and unitary isomorphisms, the conjectures relate the Weiss tower for $V \mapsto \mathrm{BU}(V)$, the spectra $A_{p^{k}}$, and a "bu-Whitehead conjecture." An interpretation of our conjectures is that our construction provides "minimal projective resolutions" of $\mathrm{H} \mathbb{Z}$ and $b u\langle 0\rangle$ (the fiber of the map $b u \rightarrow \mathrm{H} \mathbb{Z}$ ), respectively. (For $\mathrm{H} \mathbb{Z}$, this consequence is the main theorem of [7] and [9].) We further conjecture that the Taylor and Weiss towers themselves provide the contracting homotopies to establish acyclicity.

The remainder of the paper is organized as follows.

The first part of the paper sets up our general categorical construction. In Section 2, we recall Thomason's construction of homotopy colimits of permutative categories and discuss two models for homotopy pushouts. Section 3 introduces our main construction, which associates with a suitable permutative category $\mathcal{C}$ a sequence of permutative categories $\mathcal{K}_{m} \mathcal{C}$.

In the middle part of the paper, we do the main technical work for identifying the spectra resulting from our construction. In Section 4, we construct a sequence of combinatorially defined categories $\mathcal{F}_{m} \mathcal{C}$, and in Sections 5,6 , and 7 we show them to be equivalent, under mild hypotheses, to the sequence $\mathcal{K}_{m} \mathcal{C}$. In Section 8 , we show that under further hypotheses, the categories $\mathcal{F}_{m} \mathcal{C}$ can be interpreted in group-theoretic terms, in the style of [12]. We also observe that if $\mathcal{C}$ is the category of finite sets then we recover the constructions of [12]. In particular, the sequence $\mathcal{F}_{m} \mathcal{C}$ gives rise to the filtration of the Eilenberg-Mac Lane spectrum $\mathrm{H} \mathbb{Z}$ by symmetric powers of spheres.

In the last part of the paper, we focus our attention on the specific example of finite-dimensional complex vector spaces and its relationship with the example of finite sets. In Section 9, we describe the subquotients of our filtration in the unitary case in terms of the complex of direct-sum decompositions of a complex vector space introduced in [1]. Section 10 relates the complex of direct-sum decompositions to the symplectic Tits building, and uses this to establish a shifted self-duality result for the complex of direct-sum decompositions. In Section 11 we use this self-duality to show that the subquotients in our sequence of spectra are equivalent, up to a suitable shift, to the layers in certain Taylor towers. Finally, in Section 12 we give our conjectures on the relationship of the Taylor towers to our construction. We conjecture that the Taylor towers provide contracting homotopies to a "chain complex of spectra" that one obtains from our filtration, and we suggest a buanalogue of the Whitehead Conjecture.

We would like to thank the referee for a careful reading of this paper.

\section{Notation:}

If $n$ is a nonnegative integer, we write $\underline{n}$ for the set $\{1,2, \ldots, n\}$.

If $\mathcal{P}$ is a poset, then we write $|\mathcal{P}|$ for its geometric realization, i.e., the nerve of $\mathcal{P}$ regarded as a category. If $\mathcal{C}$ is a category, we write either $B \mathcal{C}$ or $|\mathcal{C}|$ for its nerve, depending on context. We also use the letter $B$ to indicate delooping.

If $\mathcal{C}$ and $\mathcal{D}$ are categories, we write $\mathcal{C} \simeq \mathcal{D}$ and say that the categories are weakly equivalent if there exists a chain of functors beginning at $\mathcal{C}$ and ending at $\mathcal{D}$, possibly going in both directions, such that the functors induce weak equivalences of spaces on the nerves. 
If $F: \mathcal{C} \rightarrow \mathcal{D}$ is a functor and $D$ is an object of $\mathcal{D}$, we write $\mathcal{C} \downarrow D$ for the category over $D$ : objects are pairs $(C, f)$ where $f$ is a morphism $F(C) \rightarrow D$ in $\mathcal{D}$, and morphisms are commuting triangles. We use repeatedly the result that if $|\mathcal{C} \downarrow D|$ is contractible for every object $D$ in $\mathcal{D}$, then $|F|$ induces a homotopy equivalence of nerves ([16], Theorem B).

Throughout the paper, when we refer to a subgroup of a Lie group, we always mean a closed subgroup.

\section{Homotopy COLimits of PERMUtATive CATEGORIES}

In this section, we recall Thomason's construction of homotopy colimits of diagrams of permutative categories [20]. Our goal is to present a slightly simplified version of his model of the homotopy pushout for use in our construction in Section 3. We also compare this construction with the bar construction, which will be needed in Section 7.

Throughout the paper, we restrict ourselves to "augmented" categories, as specified in the following definition. Let $\mathbb{Z}_{\geq 0}$ indicate the symmetric monoidal category whose objects are nonnegative integers with only identity morphisms, with addition as the monoidal operation. We use the term "monoidal functor" to mean "lax monoidal functor" in the sense of [20].

Definition 2.1. Let $\mathcal{C}$ be a symmetric monoidal category. An augmentation of $\mathcal{C}$ is a monoidal functor $\epsilon: \mathcal{C} \rightarrow \mathbb{Z}_{>0}$. We write $\mathcal{C}_{n}$ for the full subcategory of $\mathcal{C}$ whose objects are in $\epsilon^{-1}(n)$, and we call $\mathcal{C}_{n}$ the $n$th component of $\mathcal{C}$. We will say that $\mathcal{C}$ is reduced if $\mathcal{C}_{0}$ consists only of the zero object and the identity morphism.

We note that if $\mathcal{C}$ is augmented, then it is a disjoint union $\mathcal{C}=\coprod_{n>0} \mathcal{C}_{n}$. Examples of reduced augmented symmetric monoidal categories are the category of finite sets, with the augmentation given by cardinality, the category of finite-dimensional vector spaces over a field, with the augmentation given by dimension, the category of free modules over a ring possessing the dimension invariance property, with augmentation given by the dimension, and the category of free groups, with augmentation given by the number of generators.

Recall that a symmetric monoidal category is called "permutative" if the unit and associativity isomorphisms are actually identity morphisms. Any symmetric monoidal category is equivalent to a permutative category; for example, take the equivalent category that has just one object in each isomorphism class. For reasons of convenience, we will always work with permutative, rather than symmetric monoidal, categories. From now on, we assume unless otherwise stated that our categories are permutative, augmented, and reduced.

The nerve of a permutative category group-completes to an infinite loop space, and hence there is an associated spectrum. We will be interested in looking at homotopy pushouts of spectra arising in this manner, and we would like to have a categorical model for this pushout. In other words, we would like to have a homotopy pushout of permutative categories that corresponds to the homotopy pushout of spectra. Such a homotopy colimit was constructed by Thomason in $[20] .^{1}$ For the reader's convenience, we recall the general definition.

\footnotetext{
${ }^{1}$ This construction should not be confused with another, more elementary homotopy colimit of categories considered by Thomason in an earlier paper (the so-called "lax colimit"). The more
} 
Definition 2.2 ([20], Construction 3.22). Let $F$ be a functor from a small category $\mathcal{L}$ to the category of permutative categories. Then hocolim $F$ is defined to be the following category.

- The objects of hocolim $F$ have the form $\left[\left(L_{1}, X_{1}\right), \ldots,\left(L_{n}, X_{n}\right)\right]$ where $n$ is a positive integer, $L_{i}$ is an object of $\mathcal{L}$ for $i=1, \ldots, n$, and $X_{i}$ is an object of $F\left(L_{i}\right)$ for $i=1, \ldots, n$.

- A morphism $\alpha$ : $\left[\left(L_{1}, X_{1}\right), \ldots,\left(L_{n}, X_{n}\right)\right] \rightarrow\left[\left(L_{1}^{\prime}, X_{1}^{\prime}\right), \ldots,\left(L_{m}^{\prime}, X_{m}^{\prime}\right)\right]$ is given by the following data: (i) a surjection $\alpha: \underline{n} \rightarrow \underline{m}$, (ii) for each $1 \leq i \leq n$, a morphism $\beta_{i}: L_{i} \rightarrow L_{\alpha(i)}^{\prime}$ in $\mathcal{L}$, and (iii) for each $1 \leq j \leq m$, a morphism $\bigoplus_{i \in \alpha^{-1}(j)} \beta_{i}\left(X_{i}\right) \rightarrow X_{j}^{\prime}$ in $F\left(L_{j}^{\prime}\right)$.

In Thomason's words, it is "painful but straightforward" to check that this defines a category. There is a monoidal structure on hocolim $F$, given on objects by concatenation, and it makes hocolim $F$ into a permutative category.

We will use a slightly simplified version of this construction for a homotopy pushout square. Let $\mathcal{A}, \mathcal{B}$, and $\mathcal{C}$ be augmented permutative categories, and assume that $\mathcal{A} \rightarrow \mathcal{B}$ and $\mathcal{A} \rightarrow \mathcal{C}$ are monoidal functors that preserve the augmentation.

Definition 2.3. Given a diagram

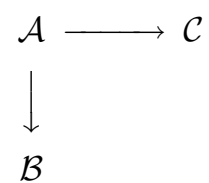

of augmented, permutative categories, we define the augmented permutative category $\mathcal{B} \otimes{ }_{\mathcal{A}} \mathcal{C}$ as follows.

(1) Objects consist of an object in $\mathcal{B}$, an object in $\mathcal{C}$, and a list of objects in $\mathcal{A}$, i.e., a nonnegative integer $n$ and a map $f: \underline{n} \cup\{b, c\} \rightarrow \operatorname{Obj}(\mathcal{A}) \cup \operatorname{Obj}(\mathcal{B}) \cup$ $\operatorname{Obj}(\mathcal{C})$ such that $f(b) \in \operatorname{Obj}(\mathcal{B}), f(c) \in \operatorname{Obj}(\mathcal{C})$, and $f(i) \in \operatorname{Obj}(\mathcal{A})$ for all $i \in \underline{n}$.

(2) If $\left[B \bar{B} ; A_{1}, \ldots, A_{n} ; C\right]$ and $\left[B^{\prime} ; A_{1}^{\prime}, \ldots, A_{k}^{\prime} ; C^{\prime}\right]$ are defined by maps $f$ and $f^{\prime}$, respectively, then morphisms $\left[B ; A_{1}, \ldots, A_{n} ; C\right] \rightarrow\left[B^{\prime} ; A_{1}^{\prime}, \ldots, A_{k}^{\prime} ; C^{\prime}\right]$ consist of:

- a surjective map $g: \underline{n} \cup\{b, c\} \rightarrow \underline{k} \cup\{b, c\}$ such that $b \mapsto b$ and $c \mapsto c$; - for all $j \in \underline{k} \cup\{b, c\}$, a morphism

$$
\oplus_{x \in g^{-1}(j)} f(x) \rightarrow f^{\prime}(j) .
$$

(3) The symmetric monoidal product $\left[B ; A_{1}, \ldots, A_{n} ; C\right] \oplus\left[B^{\prime} ; A_{1}^{\prime}, \ldots, A_{k}^{\prime} ; C^{\prime}\right]$ is given by $\left[B \oplus B^{\prime} ; A_{1}, \ldots, A_{n}, A_{1}^{\prime}, \ldots, A_{k}^{\prime} ; C \oplus C^{\prime}\right]$.

(4) The augmentation is defined by

$$
\epsilon\left[B ; A_{1}, \ldots, A_{n} ; C\right]=\epsilon(B)+\epsilon\left(A_{1}\right)+\cdots+\epsilon\left(A_{n}\right)+\epsilon(C) .
$$

Proposition 2.4. The diagram

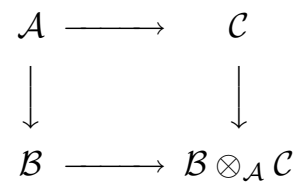

elementary homotopy colimit corresponds to a homotopy colimit of spaces, while the homotopy colimit that we are interested in corresponds to a homotopy colimit of spectra. 
passes (upon application of Segal's construction) to a homotopy pushout diagram of spectra.

Proof. Let $\mathcal{D}$ denote the category resulting from applying Thomason's hocolim construction to (2.1); upon application of Segal's construction, the square

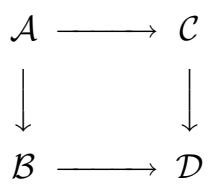

passes to a homotopy pushout diagram of spectra by [20], Theorem 4.1. Up to isomorphism, a typical object of $\mathcal{D}$ has the form $\left[B_{1}, \ldots, B_{k} ; A_{1}, \ldots, A_{m} ; C_{1}, \ldots, C_{n}\right]$, and there is a monoidal retraction $\mathcal{D} \rightarrow \mathcal{B} \otimes_{\mathcal{A}} \mathcal{C}$ by

$\left[B_{1}, \ldots, B_{k} ; A_{1}, \ldots, A_{m} ; C_{1}, \ldots, C_{n}\right] \mapsto\left[B_{1} \oplus \cdots \oplus B_{k} ; A_{1}, \ldots, A_{m} ; C_{1} \oplus \cdots \oplus C_{n}\right]$ that is compatible with the inclusions of $\mathcal{B}$ and $\mathcal{C}$. Since the over-category $\mathcal{D} \downarrow$ $\left[B ; A_{1}, \ldots, A_{m} ; C\right]$ has $\left[B ; A_{1}, \ldots, A_{m} ; C\right]$ with the identity map as a terminal object, and so has contractible nerve, we know that $\mathcal{D}$ and $\mathcal{B} \otimes_{\mathcal{A}} \mathcal{C}$ have equivalent nerves. Therefore $\mathcal{D}$ and $\mathcal{B} \otimes{ }_{\mathcal{A}} \mathcal{C}$ give rise to the same spectrum upon the application of Segal's construction, which completes the proof.

The attraction of Thomason's pushout is that it keeps one in the category of permutative categories, without introducing a simplicial dimension with each application. It is ideally suited for our general construction in Section 3. The analysis of specific examples, however, will involve the combinatorial model that we introduce in Section 4, and to establish the relationship of the model to our construction, we will need to use a different model for the pushout, namely the bar construction. Given a pushout diagram as in (2.1), the bar construction is defined to be the simplicial permutative category $[q] \mapsto \mathcal{B} \times \mathcal{A}^{q} \times \mathcal{C}$, with the evident face and degeneracy maps. Taking nerves degree-wise, one obtains a simplicial $\Gamma$-space, which we denote $\operatorname{Bar}(\mathcal{B}, \mathcal{A}, \mathcal{C})$; taking geometric realization, one again obtains a $\Gamma$-space.

We may also consider the version of the bar construction in which there are only face maps, but no degeneracy maps. Thus we represent the bar construction via the contravariant functor $[q] \mapsto \mathcal{B} \times \mathcal{A}^{q} \times \mathcal{C}$ where $[q]$ ranges over the category of standard ordered sets and injective maps that are monotonic increasing.

Proposition 2.5. There is an augmentation-preserving monoidal functor

$$
f: \operatorname{hocolim}_{q} \mathcal{B} \times \mathcal{A}^{q} \times \mathcal{C} \rightarrow \mathcal{B} \otimes_{\mathcal{A}} \mathcal{C},
$$

where hocolim is the Thomason homotopy colimit of the face maps in the bar construction. This functor induces a homotopy equivalence of $\Gamma$-spaces

$$
|f|:|\operatorname{Bar}(\mathcal{B}, \mathcal{A}, \mathcal{C})| \rightarrow\left|\mathcal{B} \otimes_{\mathcal{A}} \mathcal{C}\right| \text {. }
$$

Proof. There is an obvious inclusion functor $f_{q}: \mathcal{B} \times \mathcal{A}^{q} \times \mathcal{C} \rightarrow \mathcal{B} \otimes_{\mathcal{A}} \mathcal{C}$. This functor is monoidal, meaning that it is lax monoidal in the sense of [20], page 1593 , and therefore it passes to a $\Gamma$-map of $\Gamma$-spaces upon realization. For an injective monotonic map $\alpha:[q] \rightarrow\left[q^{\prime}\right]$, which corresponds to a face map $\partial_{\alpha}$ : $\mathcal{B} \times \mathcal{A}^{q^{\prime}} \times \mathcal{C} \rightarrow \mathcal{B} \times \mathcal{A}^{q} \times \mathcal{C}$, there is a natural transformation $f_{q^{\prime}} \rightarrow f_{q} \circ \partial_{\alpha}$. These natural transformations satisfy the conditions of [20], Proposition 3.21, and therefore the functors $f_{q}$ assemble to a monoidal functor, which we denote $f$ :

$$
f: \operatorname{hocolim}_{q} \mathcal{B} \times \mathcal{A}^{q} \times \mathcal{C} \rightarrow \mathcal{B} \otimes_{\mathcal{A}} \mathcal{C} .
$$


The geometric realization of this homotopy colimit is $\Gamma$-equivalent to $|\operatorname{Bar}(\mathcal{B}, \mathcal{A}, \mathcal{C})|$, and so we have a $\operatorname{Gamma} \operatorname{map}|f|:|\operatorname{Bar}(\mathcal{B}, \mathcal{A}, \mathcal{C})| \rightarrow\left|\mathcal{B} \otimes_{\mathcal{A}} \mathcal{C}\right|$.

To prove that $|f|$ is a homotopy equivalence, one may mimic Thomason's proofs of his main theorems. Namely, one first proves that $f$ is an equivalence when the diagram $\mathcal{B} \leftarrow \mathcal{A} \rightarrow \mathcal{C}$ is a diagram of free permutative categories and free functors, in which case it is a straightforward calculation. For the general case, one uses the fact that permutative categories have canonical free resolutions.

\section{The general COnstruction of the FiLtration}

In this section, we use the homotopy pushouts of Section 2 to construct a filtration on a general augmented permutative category $\mathcal{C}$ with associated spectrum $A$. The result is a sequence of augmented permutative categories and monoidal functors,

$$
\mathcal{C}=\mathcal{K}_{0} \mathcal{C} \rightarrow \mathcal{K}_{1} \mathcal{C} \rightarrow \cdots \rightarrow \mathcal{K}_{\infty} \mathcal{C} \simeq \mathbb{Z}_{\geq 0}
$$

and when we apply Segal's infinite loop space machine, we obtain a sequence of spectra

$$
A=A_{0} \rightarrow A_{1} \rightarrow \cdots \rightarrow \mathrm{H} \mathbb{Z}
$$

The filtration is obtained by "killing" the components of $\mathcal{C}$, one at a time. To set this up, we begin with a definition and then recall some technical results that we will need.

Definition 3.1. Let $\mathcal{C}$ be a small category. The free unital permutative category on $\mathcal{C}$, denoted Free $(\mathcal{C})$, is the category whose objects are $(n, f)$ where $n$ is a positive integer and $f: \underline{n} \rightarrow \operatorname{Obj}(\mathcal{C})$. There are morphisms from $(n, f)$ to $(k, g)$ only if $n=k$; a morphism $(n, f) \rightarrow(n, g)$ is given by (i) an isomorphism $\sigma: \underline{n} \rightarrow \underline{n}$, and (ii) for each $i \in \underline{n}$, a morphism $f(i) \rightarrow g(\sigma i)$ in $\mathcal{C}$. In addition, there is a unit object $\underline{0}$.

In brief

$$
\operatorname{Free}(\mathcal{C}):=\coprod_{n \geq 0} \mathcal{C} \prec \Sigma_{n}
$$

The following lemma and proposition are routine.

\section{Lemma 3.2.}

(1) $\operatorname{Free}(\mathcal{C})$ is a permutative category.

(2) Given a permutative category $\mathcal{D}$ and a functor $F: \mathcal{C} \rightarrow \mathcal{D}$, there is a unique extension of $F$ to a monoidal functor $\operatorname{Free}(\mathcal{C}) \rightarrow \mathcal{D}$.

Proposition 3.3 ([20], Lemma 2.5). The spectrum associated to the symmetric monoidal category $\operatorname{Free}(\mathcal{C})$ is $\Sigma^{\infty} B \mathcal{C}_{+}$.

Given an augmented permutative category $\mathcal{C}$ whose $m$ th component is $\mathcal{C}_{m}$, we want to construct an augmented permutative category that will play the role of the quotient of $\mathcal{C}$ by $\mathcal{C}_{m}$. To this end, endow $\operatorname{Free}\left(\mathcal{C}_{m}\right)$ with the augmentation $\epsilon(n, f)=m n$, so that the functor $\operatorname{Free}\left(\mathcal{C}_{m}\right) \rightarrow \mathcal{C}$ extending the inclusion is augmentation preserving. Let $\mathcal{S}$ denote the category of finite sets and isomorphisms. There is a natural projection $\operatorname{Free}\left(\mathcal{C}_{m}\right) \rightarrow \mathcal{S}$, and in order for the projection to preserve augmentation, we endow $\mathcal{S}$ with the augmentation $\epsilon(S)=m|S|$ and call the resulting augmented category $\mathcal{S}_{m}$. Recall that the tensor product of categories is defined in Definition 2.3 . 
Definition 3.4. The homotopy cofiber of the map $\operatorname{Free}\left(\mathcal{C}_{m}\right) \rightarrow \mathcal{C}$, is the augmented permutative category $\mathcal{C} / / \mathcal{C}_{m}:=\mathcal{S}_{m} \otimes_{\operatorname{Free}\left(\mathcal{C}_{m}\right)} \mathcal{C}$.

We think of $\mathcal{C} / / \mathcal{C}_{m}$ as "the category obtained from $\mathcal{C}$ by killing the $m$ th component." This is justified by the following lemma.

Lemma 3.5. The inclusion functor $\mathcal{C} \rightarrow \mathcal{C} / / \mathcal{C}_{m}$ is an isomorphism on components of augmentation less than $m$. The $m$ th component of $\mathcal{C} / / \mathcal{C}_{m}$ has contractible nerve.

Proof. Let $\mathcal{D}=\mathcal{C} / / \mathcal{C}_{m}$. We already know that $\mathcal{D}$ is an augmented permutative category. Objects in $\mathcal{D}$ have one of the following forms:

- $D=\left[\underline{n} ;\left(n_{1}, f_{1}\right),\left(n_{2}, f_{2}\right), \ldots,\left(n_{k}, f_{k}\right) ; C\right]$, where each $n_{i}$ is a positive integer, $f_{i}$ is a map from $\underline{n}_{i}$ to the objects of $\mathcal{C}_{m}$, and $C$ is an arbitrary object in $\mathcal{C}$;

- $D=[\underline{n} ; \underline{0} ; C]$, where $\underline{0}$ is the unit object in $\operatorname{Free}\left(\mathcal{C}_{m}\right)$ and $C$ is an arbitrary object in $\mathcal{C}$.

Suppose that $i<m$; we must prove that $\mathcal{C}_{i} \cong \mathcal{D}_{i}$. For an object $D$ to have augmentation $i$, it must have the second form and also have $n=0$, because otherwise a nonzero multiple of $m$ would be contributed to the augmentation of $D$. Thus such an object must have the form $[\underline{0} ; \underline{0} ; C]$, where $\epsilon(C)=i$. The full subcategory of such objects is isomorphic to $\mathcal{C}_{i}$.

We must also show that $\left|\mathcal{D}_{m}\right| \simeq *$. There are three types of objects of $\mathcal{D}$ that have augmentation equal to $m$.

(1) $[\underline{1} ; \underline{0} ; \underline{0}]$, since $\underline{1} \in \mathcal{S}_{m}$ has augmentation $m$;

(2) $[\underline{0} ;(\underline{1}, 1 \mapsto C) ; \underline{0}]$, where $C \in \mathcal{C}_{m}$ and so has augmentation $m$. We will, until the end of this proof, denote such an object more simply as $[\underline{0} ; C ; \underline{0}]$;

(3) $[\underline{0} ; \underline{0} ; C]$, where $C \in \mathcal{C}_{m}$.

There are five types of morphisms, which we categorize by the type of the object in the target.

(1) (a) $[\underline{1} ; \underline{0} ; \underline{0}]$ has only the identity self-map; it has no morphisms to objects of other types.

(b) For each object $C \in \mathcal{C}_{m}$, there is a unique morphism

$$
[\underline{0} ; C ; \underline{0}] \rightarrow[\underline{1} ; \underline{0} ; \underline{0}] .
$$

(2) For each morphism $C \rightarrow C^{\prime}$ in $\mathcal{C}_{m}$, there is a morphism

$$
[\underline{0} ; C ; \underline{0}] \rightarrow\left[\underline{0} ; C^{\prime} ; \underline{0}\right] .
$$

(3) For each morphism $C \rightarrow C^{\prime}$ in $\mathcal{C}_{m}$, there are morphisms

$$
\begin{aligned}
& {[\underline{0} ; C ; \underline{0}] \rightarrow\left[\underline{0} ; \underline{0} ; C^{\prime}\right]} \\
& {[\underline{0} ; \underline{0} ; C] \rightarrow\left[\underline{0} ; \underline{0} ; C^{\prime}\right] .}
\end{aligned}
$$

We define the following subcategories of $\mathcal{D}_{m}$. Let $\mathcal{D}_{m}^{1}$ be the full subcategory of objects of the form $[\underline{1} ; \underline{0} ; \underline{0}]$ or $[\underline{0} ; C ; \underline{0}]$, let $\mathcal{D}_{m}^{2}$ be the full subcategory of objects of the form $[\underline{0} ; C ; \underline{0}]$ or $\left[\underline{0} ; \underline{0} ; C^{\prime}\right]$, and let $\mathcal{D}_{m}^{12}=\mathcal{D}_{m}^{1} \cap \mathcal{D}_{m}^{2}$ be the full subcategory of objects of the form $[\underline{0} ; C ; \underline{0}]$. The nerve of $\mathcal{D}_{m}$ is the union of the nerve of $\mathcal{D}_{m}^{1}$ and the nerve of $\mathcal{D}_{m}^{2}$. Since the inclusion $\left|\mathcal{D}_{m}^{12}\right| \hookrightarrow\left|\mathcal{D}_{m}^{1}\right|$ is a cofibration, it follows that there is a homotopy pushout square

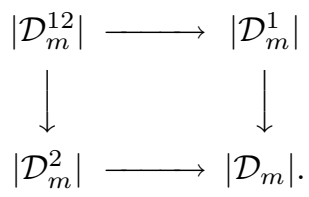


Contractibility of $\left|\mathcal{D}_{m}\right|$ now follows from two claims: $\left|\mathcal{D}_{m}^{1}\right|$ is contractible, and the map $\left|\mathcal{D}_{m}^{12}\right| \rightarrow\left|\mathcal{D}_{m}^{2}\right|$ is a homotopy equivalence. For the first claim, note that $\mathcal{D}_{m}^{1}$ has $[\underline{1} ; \underline{0} ; \underline{0}]$ as a final object. For the second claim, consider the functor $\Psi: \mathcal{D}_{m}^{2} \rightarrow \mathcal{D}_{m}^{12}$ defined on objects by $\Psi([0 ; C ; 0])=[0 ; C ; 0]$ and $\Psi([0 ; 0 ; C])=[0 ; C ; 0]$. To define $\Psi$ on morphisms, send morphisms of type (2) or (3) to the corresponding morphisms of type (2). Then $\Psi$ provides a homotopy inverse to the inclusion $\left|\mathcal{D}_{m}^{12}\right| \rightarrow\left|\mathcal{D}_{m}^{2}\right|$.

Definition 3.6. We say that an augmented permutative category $\mathcal{C}$ is " $m$-reduced" if $\mathcal{C}$ is reduced and $\left|\mathcal{C}_{i}\right| \simeq *$ for $i \leq m$.

We have the following corollary.

Corollary 3.7. If $\mathcal{C}$ is $(m-1)$-reduced, then $\mathcal{C} / / \mathcal{C}_{m}$ is m-reduced.

Construction 3.8. Let $\mathcal{C}$ be a reduced augmented permutative category with associated spectrum $A$. We define a sequence of permutative categories $\mathcal{K}_{m} \mathcal{C}$ inductively, beginning with $\mathcal{K}_{0} \mathcal{C}=\mathcal{C}$. Once $\mathcal{K}_{m-1} \mathcal{C}$ has been defined, let $\mathcal{K}_{m-1} \mathcal{C}_{m}$ be the $m$ th component of $\mathcal{K}_{m-1} \mathcal{C}$, and define $\mathcal{K}_{m} \mathcal{C}=\mathcal{K}_{m-1} \mathcal{C} / / \mathcal{K}_{m-1} \mathcal{C}_{m}$. It follows immediately from Corollary 3.7 and induction that $\mathcal{K}_{m} \mathcal{C}$ is $m$-reduced. There are inclusion functors $\mathcal{K}_{m-1} \mathcal{C} \rightarrow \mathcal{K}_{m} \mathcal{C}$. Letting $\mathcal{K}_{\infty} \mathcal{C}$ be the colimit of the construction, we obtain a sequence

$$
\mathcal{C}=\mathcal{K}_{0} \mathcal{C} \rightarrow \mathcal{K}_{1} \mathcal{C} \rightarrow \cdots \rightarrow \mathcal{K}_{\infty} \mathcal{C} \simeq \mathbb{Z}_{\geq 0}
$$

and when we apply Segal's infinite loop space machine, we obtain a sequence of spectra

$$
A=A_{0} \rightarrow A_{1} \rightarrow \cdots \rightarrow \mathrm{H} \mathbb{Z}
$$

This is our filtration of the spectrum $A$ associated to $\mathcal{C}$. Strictly speaking, it is a relative filtration interpolating between $A$ and $\mathrm{H} \mathbb{Z}$. The following theorem follows immediately from the definition of the categories $\mathcal{K}_{m} \mathcal{C}$, Proposition 2.4, and Proposition 3.3.

Theorem 3.9. Let $\Sigma^{\infty}\left(B \mathcal{K}_{m-1} \mathcal{C}_{m}\right)_{+} \rightarrow A_{m-1}$ be induced by extending the inclusion $\mathcal{K}_{m-1} \mathcal{C}_{m} \rightarrow \mathcal{K}_{m-1} \mathcal{C}$ to a functor Free $\left(\mathcal{K}_{m-1} \mathcal{C}_{m}\right) \rightarrow \mathcal{K}_{m-1} \mathcal{C}$. Let the map $\epsilon: \Sigma^{\infty}\left(B \mathcal{K}_{m-1} \mathcal{C}_{m}\right)_{+} \rightarrow \mathbf{S}$ be induced by the map $B \mathcal{K}_{m-1} \mathcal{C}_{m} \rightarrow *$. Then

$$
A_{m} \simeq \operatorname{hocolim}\left(\begin{array}{c}
\sum^{\infty}\left(B \mathcal{K}_{m-1} \mathcal{C}_{m}\right)_{+} \longrightarrow A_{m-1} \\
\epsilon \downarrow \\
\mathbf{S}
\end{array}\right)
$$

Thus by construction the subquotient $A_{m} / A_{m-1}$ is equivalent to $\Sigma^{\infty} \Sigma B \mathcal{K}_{m-1} \mathcal{C}_{m}$, and so the subquotients of our filtration are suspension spectra.

\section{Blowing up $\mathcal{C}$ TO $\mathcal{F}_{\infty} \mathcal{C}$}

In this section, we begin the development of a simplified combinatorial model for the sequence of categories described in Construction 3.8. In the case that $\mathcal{C}$ is a "monogenic" category (Definition 4.1 ), we embed $\mathcal{C}$ in a much larger category $\mathcal{F}_{\infty} \mathcal{C}$ that is monoidally equivalent to $\mathbb{Z}_{\geq 0}$. Then in Sections 5,6 , and 7 , we prove that if $\mathcal{C}$ satisfies a mild condition (Axiom 5.1), then a certain filtration of $\mathcal{F}_{\infty} \mathcal{C}$ models Construction 3.8, with our main goal being Theorem 7.1. In Section 8 we show that further assumptions allow us to recast our combinatorial description in group-theoretic terms, as in [12]. 
Our goal in this section is to define the category $\mathcal{F}_{\infty} \mathcal{C}$ and to study the morphism spaces, both through examples and by proving Lemma 4.12 , which says that $\mathcal{F}_{\infty} \mathcal{C}$ can be thought of as a category of epimorphisms. We will use a category $\mathcal{C}$ that consists of isomorphisms of an underlying category $\mathcal{B}$ that is itself generated under coproducts by a single object, as detailed in the following definition.

Definition 4.1. Let $\mathcal{B}$ be a (possibly topological) category with strictly associative coproduct.

(1) We call $\mathcal{B}$ pointed if it has a zero object, $\underline{0}$, that is both initial and final.

(2) We call a pointed category $\mathcal{B}$ monogenic if it has an object $g$ (the generator) such that the objects of $\mathcal{B}$ consist of $\underline{0}$ and the coproducts of $g$ with itself $n$ times for $n=1,2,3 \ldots$. In this case, we write $g^{n}$ for the objects of $\mathcal{B}$, where $g^{0}$ means the zero object, and we give $\mathcal{B}$ the permutative structure arising from the coproduct. We require that a monogenic category $\mathcal{B}$ have the property of "dimension invariance," namely that $g^{n} \cong g^{r}$ implies $n=r$.

We will be particularly interested in the category of finite-dimensional complex vector spaces (generated by $\mathbb{C}$ ). Other examples of interest are the category of finite pointed sets (generated by $\underline{1}$ ), the category of finitely-generated free modules over a ring $R$ that has the property of dimension invariance (generated by $R$ ), and the category of finitely-generated free groups (generated by $\mathbb{Z}$ ). In each case, we force the coproducts to be strictly associative by taking the equivalent category that has only one object in each isomorphism class.

We need to consider certain subcategories of isomorphisms in $\mathcal{B}$.

Definition 4.2. Let $\mathcal{B}$ be a monogenic category. A category of admissible isomorphisms in $\mathcal{B}$ is a subcategory $\mathcal{C}$ satisfying the following conditions.

(1) $\operatorname{Obj}(\mathcal{C})=\operatorname{Obj}(\mathcal{B})$.

(2) Morphisms in $\mathcal{C}$ are isomorphisms in $\mathcal{B}$, and $\mathcal{C}$ is closed under inverses.

(3) All the automorphisms of $g^{n}$ induced by permutations are in $\mathcal{C}$.

(4) Let $f: A \rightarrow B$ and $g: C \rightarrow D$ be morphisms in $\mathcal{B}$. Then $f$ and $g$ are both in $\mathcal{C}$ if and only if the morphism $f \oplus g: A \oplus C \rightarrow B \oplus D$ is in $\mathcal{C}$.

We call the morphisms of $\mathcal{C}$ the $\mathcal{C}$-admissible isomorphisms in $\mathcal{B}$. We give the category $\mathcal{C}$ the permutative structure inherited from $\mathcal{B}$ and the augmentation $\epsilon\left(g^{n}\right)=n$. By abuse of terminology, we also call $\mathcal{C}$ a monogenic category.

For the remainder of this section, let $\mathcal{B}$ be a monogenic category, as above, and let $\mathcal{C}$ be a subcategory of admissible isomorphisms. In most specific cases that we consider in this paper, $\mathcal{C}$ is the full subcategory of isomorphisms of $\mathcal{B}$. The only exception is the category of real or complex vector spaces. In this case, we endow the vector spaces with a positive-definite inner product and let $\mathcal{C}$ be the category of isometric isomorphisms.

The category $\mathcal{C}$ is a category of isomorphisms, in other words a groupoid, and since each isomorphism class of $\mathcal{C}$ has just one object, we can write $\mathcal{C}$ as a disjoint union of groups, namely $\coprod_{n} \operatorname{Aut}_{\mathcal{C}}\left(g^{n}\right)$. We use the notation $G_{n}$ for the (possibly topological) group $\operatorname{Aut}_{\mathcal{C}}\left(g^{n}\right)$. For example, in the case that $\mathcal{B}$ is the category of finite-dimensional complex vector spaces and $\mathcal{C}$ consists of the unitary isomorphisms, we have $G_{n}=U(n)$, and $\mathcal{C}=\bigsqcup_{n} U(n)$, the disjoint union of unitary groups. 
Although the category $\mathcal{F}_{\infty} \mathcal{C}$ will be constructed out of isomorphisms in $\mathcal{C}$, we will also need some of the structure of the underlying category $\mathcal{B}$. We follow Waldhausen for the definition of cofibration.

Definition 4.3. A morphism $i: V \rightarrow W$ in $\mathcal{B}$ is a $\mathcal{C}$-cofibration if there exists an object $V^{\prime}$ and a $\mathcal{C}$-admissible isomorphism $V \oplus V^{\prime} \cong W$ such that $i$ is the composite $V=V \oplus \underline{0} \rightarrow V \oplus V^{\prime} \cong W$. A subobject of $W \in \operatorname{Obj}(\mathcal{B})$ is an equivalence class of cofibrations $V \rightarrow W$ under the equivalence relation of precomposition by a $\mathcal{C}$-admissible isomorphism.

Typically, the category $\mathcal{C}$ will be clear from context, and we simply refer to "cofibrations in $\mathcal{B}$." We often use the notation $V \hookrightarrow W$ for a cofibration.

Remark 4.4. The group $\operatorname{Aut}_{\mathcal{C}}(W)$ acts transitively on the set (or space) of cofibrations $V \hookrightarrow W$ in the following sense: if $i_{1}, i_{2}$ are two cofibrations from $V$ to $W$, then there exists an element $\tau \in \operatorname{Aut}_{\mathcal{C}}(W)$ such that $i_{2}=\tau \circ i_{1}$.

In the case of finite-dimensional real or complex vector spaces, the cofibrations are linear isometric inclusions, and the subobjects correspond, as one expects, to vector subspaces. In the case of finite sets, cofibrations are simply inclusions, and a subobject is the same as a subset. In the case of finitely-generated free modules, cofibrations are Quillen's "admissible monomorphisms," and two admissible monomorphisms represent the same subobject if and only if they have the same image, in the usual sense of the word.

The following definition gives the combinatorial construction that we will filter to obtain combinatorial models for the categories $\mathcal{K}_{m} \mathcal{C}$ defined in Construction 3.8. We will work extensively with this construction in Sections 5-8.

Definition 4.5. Let $\mathcal{B}$ and $\mathcal{C}$ be as above. The reduced, augmented, permutative category $\mathcal{F}_{\infty} \mathcal{C}$ is defined as follows.

(1) Objects of $\mathcal{F}_{\infty} \mathcal{C}$ have the form $\left[n ; n_{1}, \ldots, n_{t}\right]$, where $n$ and $t$ are nonnegative integers, and $n_{1}, \ldots, n_{t}$ are positive integers. The augmentation is given by $\epsilon\left(\left[n ; n_{1}, \ldots, n_{t}\right]\right)=n+\sum n_{i}$.

(2) There are morphisms $\left[n ; n_{1}, \ldots, n_{t}\right] \rightarrow\left[r ; r_{1}, \ldots, r_{u}\right]$ only between objects with the same augmentation. A morphism $[f]$ is given by an equivalence class of the following data.

(a) A function $\alpha_{f}: \underline{t} \rightarrow \underline{u}$.

(b) A $\mathcal{C}$-admissible isomorphism

$$
f: g^{n} \oplus g^{n_{1}} \oplus \cdots \oplus g^{n_{t}} \rightarrow g^{r} \oplus g^{r_{1}} \oplus \cdots \oplus g^{r_{u}}
$$

such that

(i) for $1 \leq j \leq u$, the composite

$$
\oplus_{i \in \alpha^{-1}(j)} g^{n_{i}} \hookrightarrow g^{n} \oplus g^{n_{1}} \oplus \cdots \oplus g^{n_{t}} \rightarrow g^{r} \oplus g^{r_{1}} \oplus \cdots \oplus g^{r_{u}}
$$

factors as a composite

$$
\oplus_{i \in \alpha^{-1}(j)} g^{n_{i}} \hookrightarrow g^{r_{j}} \hookrightarrow g^{r} \oplus g^{r_{1}} \oplus \cdots \oplus g^{r_{u}}
$$

where the first map is a $\mathcal{C}$-cofibration and the second map is the standard inclusion;

(ii) if we define $e_{j}=r_{j}-\sum_{i \in \alpha^{-1}(j)} n_{i}$, then the composite

$$
g^{n} \hookrightarrow g^{n} \oplus g^{n_{1}} \oplus \cdots \oplus g^{n_{t}} \rightarrow g^{r} \oplus g^{r_{1}} \oplus \cdots \oplus g^{r_{u}}
$$


factors through the composite of a $\mathcal{C}$-admissible isomorphism $\theta_{f}: g^{n} \rightarrow g^{r} \oplus g^{e_{1}} \oplus \cdots \oplus g^{e_{u}}$ and a coproduct of the identity on $g^{r}$ with $u$ cofibrations in $\mathcal{B}$,

$$
g^{r} \oplus g^{e_{1}} \oplus \cdots \oplus g^{e_{u}} \hookrightarrow g^{r} \oplus g^{r_{1}} \oplus \cdots \oplus g^{r_{u}} .
$$

We call $e_{j}$ the $j$ th excess of $f$, and when necessary for specificity, we also use the notation $e\left(r_{j}\right)$, or even $e_{[f]}\left(r_{j}\right)$.

We give the name compatible with $\alpha_{f}$ to isomorphisms

$$
g^{n} \oplus g^{n_{1}} \oplus \cdots \oplus g^{n_{t}} \rightarrow g^{r} \oplus g^{r_{1}} \oplus \cdots \oplus g^{r_{u}}
$$

satisfying 2(b)(i) and 2(b)(ii). A morphism in $\mathcal{F}_{\infty} \mathcal{C}$ from $\left[n ; n_{1}, \ldots, n_{t}\right]$ to $\left[r ; r_{1}, \ldots, r_{u}\right]$ consists of a function $\alpha_{f}$ together with an equivalence class of $\mathcal{C}$-admissible isomorphisms that are compatible with $\alpha_{f}$. The equivalence relation is given by the action of $\prod G_{r_{j}}$ on the set of $\mathcal{C}$ admissible isomorphisms $g^{n} \oplus g^{n_{1}} \oplus \cdots \oplus g^{n_{t}} \rightarrow g^{r} \oplus g^{r_{1}} \oplus \cdots \oplus g^{r_{u}}$, where, as usual, $G_{r_{j}}=\operatorname{Aut}_{\mathcal{C}}\left(g^{r_{j}}\right)$.

(3) The monoidal structure on $\mathcal{F}_{\infty}(\mathcal{C})$ is defined by

$$
\left[n ; n_{1}, \ldots, n_{t}\right] \oplus\left[r ; r_{1}, \ldots, r_{u}\right]=\left[n+r ; n_{1} \ldots, n_{t}, r_{1}, \ldots, r_{u}\right],
$$

with $[0 ; \emptyset]$ as the unit.

In general, we think of an object $\left[n ; n_{1}, \ldots, n_{t}\right]$ of $\mathcal{F}_{\infty} \mathcal{C}$ as representing the object $g^{n+n_{1}+\cdots+n_{t}}$ together with a partial partition into "disjoint" or "orthogonal" subobjects of dimensions $n_{1}, \ldots, n_{t}$. Once a piece is split off into the partition, we remember only its dimension. We think of $g^{n}$ as the "free" part of the object. A morphism between two objects can combine split-off subobjects (Condition 2(b)(i)), and possibly enlarge them to larger split-off subobjects by taking a further subobject from the free part to combine into a larger subobject (Condition 2(b)(ii)).

We think of $\mathcal{F}_{\infty} \mathcal{C}$ as a model for $\mathrm{H} \mathbb{Z}$, which is justified by the following easy proposition.

Proposition 4.6. The augmentation $\epsilon: \mathcal{F}_{\infty} \mathcal{C} \rightarrow \mathbb{Z}_{\geq 0}$ induces a homotopy equivalence of classifying spaces.

Proof. The full subcategory of $\mathcal{F}_{\infty} \mathcal{C}$ consisting of objects of augmentation $n$ has $[0 ; n]$ as a terminal object.

Filtering $\mathcal{F}_{\infty}$ according to the following definition gives us combinatorially defined categories that turn out to be models for the inductively defined categories $\mathcal{K}_{m} \mathcal{C}$ and are easier to analyze.

Definition 4.7. Let $\mathcal{F}_{m} \mathcal{C}$ be the full subcategory of $\mathcal{F}_{\infty} \mathcal{C}$ whose objects are of the form $\left[n ; n_{1}, \ldots, n_{s}\right]$ such that $n_{i} \leq m$ for all $i$. (There is no restriction on $n$.)

It is clear that each $\mathcal{F}_{m} \mathcal{C}$ is a monoidal augmented subcategory of $\mathcal{F}_{\infty} \mathcal{C}$, and that there are monoidal inclusion functors $\mathcal{F}_{m-1} \mathcal{C} \rightarrow \mathcal{F}_{m} \mathcal{C}$. Our goal in subsequent sections is to prove that under suitable assumptions, the classifying space of the category $\mathcal{F}_{m} \mathcal{C}$ is $\Gamma$-equivalent to that of the category $\mathcal{K}_{m} \mathcal{C}$ (Theorem 7.1 ). We will prove it by showing that the categories $\mathcal{F}_{m-1} \mathcal{C}$ and $\mathcal{F}_{m} \mathcal{C}$ are related in the same way as $\mathcal{K}_{m-1} \mathcal{C}$ and $\mathcal{K}_{m} \mathcal{C}$ are. It will follow that the sequence of spectra arising from the sequence of categories $\mathcal{F}_{0} \mathcal{C}=\mathcal{C} \rightarrow \mathcal{F}_{1} \mathcal{C} \rightarrow \mathcal{F}_{2} \mathcal{C} \rightarrow \ldots$ is equivalent to the filtration $A=A_{0} \rightarrow A_{1} \rightarrow A_{2} \ldots$ given by Construction 3.8. 
We conclude this section by giving examples of morphism sets in $\mathcal{F}_{\infty}$, and we describe the relationship of our construction to an idea of Neil Strickland.

Remark 4.8. Note that because of the compatibility condition, a morphism $[f]$ is completely determined by the map $\alpha_{f}$ and the equivalence class of the isomorphism $\theta_{f}: g^{n} \rightarrow g^{r} \oplus g^{e_{1}} \oplus \cdots \oplus g^{e_{u}}$, where two such isomorphisms give rise to the same morphism in $\mathcal{F}_{\infty} \mathcal{C}$ exactly when they are in the same orbit of the group $G_{e_{1}} \times \cdots \times G_{e_{u}}$ acting by postcomposition.

Examples 4.9. Suppose we take $\mathcal{B}$ to be the category of finite-dimensional complex vector spaces $\mathbb{C}^{n}$ and isometric inclusions, so that $\mathcal{C}=\coprod_{n} U(n)$.

(1) The object $[n ; \emptyset]$ has automorphisms given by $G_{n}=\operatorname{Aut}_{\mathcal{C}}\left(\mathbb{C}^{n}\right)$, which in this case is $U(n)$, and there are no morphisms $[m ; \emptyset] \rightarrow[n ; \emptyset]$ if $n \neq m$. In fact, in general we have an embedding of the category $\mathcal{C}$ in $\mathcal{F}_{\infty} \mathcal{C}$ as the full subcategory with objects $[m ; \emptyset]$, that is, as $\mathcal{F}_{0} \mathcal{C}$.

(2) Consider the space of morphisms $[f]:[3 ; 1,2] \rightarrow[1 ; 2,3]$, which has three connected components, corresponding to three possible functions $\alpha_{f}$. (See Remark 4.11 about the topology we put on $\mathcal{F}_{m} \mathcal{C}$ when $\mathcal{C}$ is a topological category.)

If $\alpha_{f}(1)=1$ and $\alpha_{f}(2)=2$, then we need an equivalence class of isometric isomorphisms

$$
f: \mathbb{C}^{3} \oplus \mathbb{C}^{1} \oplus \mathbb{C}^{2} \rightarrow \mathbb{C}^{1} \oplus \mathbb{C}^{2} \oplus \mathbb{C}^{3}
$$

under the action of $U(2) \times U(3)$. Since $\alpha(1)=1$, the map $f$ is a cofibration from $\mathbb{C}^{1}$ on the left to $\mathbb{C}^{2}$ on the right, and the choice of cofibration is immaterial because of the equivalence relation. Similar consequences follow from $\alpha(2)=2$, so the equivalence class of $f$ is actually determined by what happens on $\mathbb{C}^{3}$, i.e., by the equivalence classes of isomorphisms

$$
\theta_{f}: \mathbb{C}^{3} \rightarrow \mathbb{C}^{1} \oplus \mathbb{C}^{1} \oplus \mathbb{C}^{1}
$$

under the action of $U(1) \times U(1)$ on the last two factors. Thus we obtain $U(3) /(U(1) \times U(1))$ for this connected component of the morphism space.

There are two more connected components, corresponding to $\alpha(1)=$ 2 and $\alpha(2)=1$, and $\alpha(1)=\alpha(2)=2$. Each of these components is homeomorphic to $U(3) / U(2)$.

Altogether, $\operatorname{Morph}_{\mathcal{F}_{\infty} \mathcal{C}}([3 ; 1,2],[1 ; 2,3])$ is homeomorphic to the space of $U(6)$-equivariant maps from $U(6) /(U(1) \times U(2))$ to $U(6) /(U(2) \times U(3))$. We will see in Section 8 that this is a part of a general theorem.

(3) Consider the endomorphisms $[f]$ of $[4 ; 1,1,1,5,5]$, an object of augmentation 17. The function $\alpha_{f}$ must consist of an element of $\Sigma_{3} \times \Sigma_{2}$, permuting the 1 's and the 5's, and the values $e_{1}, \ldots, e_{5}$ are all zero. Similar considerations to those above tell us that endomorphisms of $[4 ; 1,1,1,5,5]$ are all automorphisms, and correspond to elements of $U(4) \times \Sigma_{3} \times \Sigma_{2}$. This is related to the fact that the Weyl group in $U(17)$ of $U(1)^{3} \times U(5)^{2}$ is $U(4) \times \Sigma_{3} \times \Sigma_{2}$.

Example 4.10. Let $\mathcal{B}$ be the category of finite pointed sets and $\mathcal{C}$ the subcategory of isomorphisms of $\mathcal{B}$. Let $\mathcal{M}$ be the full subcategory of $\mathcal{F}_{\infty} \mathcal{C}$ with objects of the form $\left[0 ; n_{1}, \ldots, n_{k}\right]$. The nerves of $\mathcal{M}$ and $\mathcal{F}_{\infty} \mathcal{C}$ are equivalent as $\Gamma$-spaces. Indeed, the functor $\mathcal{F}_{\infty} \mathcal{C} \rightarrow \mathcal{M}$ that sends $\left[n ; n_{1}, \ldots, n_{k}\right]$ to $[0 ; \underbrace{1, \ldots, 1}_{n}, n_{1}, \ldots, n_{k}]$ 
induces a homotopy inverse to the map of classifying spaces induced by the inclusion $\mathcal{M} \rightarrow \mathcal{F}_{\infty} \mathcal{C}$.

The category $\mathcal{M}$ can be interpreted as a category of "sets with multiplicities," a definition proposed by Neil Strickland. A set with multiplicities is a finite set where every element is assigned a positive integer as its multiplicity. A morphism $f: S \rightarrow T$ is a map of sets such that for every $t \in T$, the multiplicities of the elements of $f^{-1}(t)$ add up to the multiplicity of $t$. The category $\mathcal{M}$ is isomorphic to the category of sets with multiplicities, with $\left[0 ; n_{1}, \ldots, n_{k}\right]$ corresponding to the set with $k$ elements, of multiplicities $n_{1}, \ldots, n_{k}$. Similarly, the category $\mathcal{F}_{m} \mathcal{C}$ of Definition 4.7 is equivalent to the category of sets with muliplicities at most $m$.

Remark 4.11. Notice that the morphism set

$$
\operatorname{Morph}_{\mathcal{F}_{\infty} \mathcal{C}}\left(\left[n ; n_{1}, \ldots, n_{t}\right],\left[r ; r_{1}, \ldots, r_{u}\right]\right)
$$

is a subset of

$$
\operatorname{Morph}_{\mathcal{C}}\left(g^{n+n_{1}+\cdots+n_{t}}, g^{r+r_{1}+\cdots+r_{u}}\right)_{\prod G_{r_{j}}} \cong G_{r+r_{1}+\cdots+r_{u}} /\left(\prod G_{r_{j}}\right) .
$$

If $\mathcal{B}$ is a topological category, i.e., if $G_{r}$ is a topological group, then we make $\mathcal{F}_{\infty} \mathcal{C}$ a topological category as well, by endowing $\operatorname{Morph}_{\mathcal{F}_{\infty} \mathcal{C}}\left(\left[n ; n_{1}, \ldots, n_{t}\right],\left[r ; r_{1}, \ldots, r_{u}\right]\right)$ with the subspace topology. Notice that if we set $G=G_{n+n_{1}+\cdots+n_{t}}=G_{r+r_{1}+\cdots+r_{u}}$, $G_{n_{\bullet}}=G_{n_{1}} \times \cdots \times G_{n_{t}}$, and $G_{r_{\bullet}}=G_{r_{1}} \times \cdots \times G_{r_{u}}$, then there is an inclusion of subspaces

$$
\operatorname{Morph}_{\mathcal{F}_{\infty} \mathcal{C}}\left(\left[n ; n_{1}, \ldots, n_{t}\right],\left[r ; r_{1}, \ldots, r_{u}\right]\right) \subset \operatorname{map}_{G}\left(G / G_{n}, G / G_{r_{\bullet}}\right)=\left(G / G_{r_{\bullet}}\right)^{G_{n}}
$$

In Section 8 , we will see that under some further assumptions on $\mathcal{C}$, this inclusion is in fact an isomorphism, in other words $\mathcal{F}_{\infty} \mathcal{C}$ can be identified with a union of orbit categories.

We close this section with Lemma 4.12, which can be interpreted to say that $\mathcal{F}_{\infty} \mathcal{C}$ is a "category of epimorphisms."

Lemma 4.12. Suppose we are given a diagram

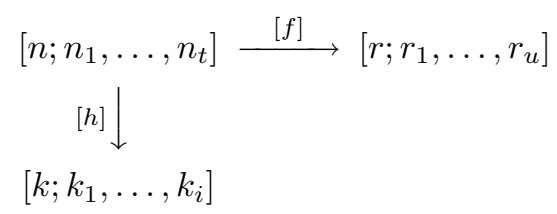

in $\mathcal{F}_{\infty} \mathcal{C}$. Then there exists at most one morphism $[p]:\left[r ; r_{1}, \ldots, r_{u}\right] \rightarrow\left[k ; k_{1}, \ldots, k_{i}\right]$ in $\mathcal{F}_{\infty} \mathcal{C}$ such that $[p] \circ[f]=[h]$.

Proof. Suppose there exists a morphism $[p]$ in $\mathcal{F}_{\infty} \mathcal{C}$ such that $[p] \circ[f]=[h]$. We can choose representative isomorphisms $p, f$, and $h$ in $\mathcal{C}$ for $[p],[f]$, and $[h]$, respectively, such that $p \circ f=h$. The choice of $p$ is determined by the choices of $f$ and $h$, since all three maps are isomorphisms in $\mathcal{C}$. However, it is conceivable that picking new representatives $f_{1}$ and $h_{1}$ of $[f]$ and $[h]$, respectively, will lead to a new map $p_{1}=h_{1} \circ f_{1}^{-1}$ with $[p] \neq\left[p_{1}\right]$. Thus we need to show that if $f_{1}$ and $h_{1}$ are any other representatives of $[f]$ and $[h]$, and if $p_{1}$ has the property that $p_{1} \circ f_{1}=h_{1}$, then $\left[p_{1}\right]=[p]$.

We begin with $h_{1}$. By definition, $\left[h_{1}\right]=[h]$ if and only if there exists $\tau \in$ $G_{k_{1}} \times \cdots \times G_{k_{i}}$ such that $h_{1}=\tau \circ h$, in which case we also have $h_{1} \circ f^{-1}=\tau \circ h \circ f^{-1}$. Thus $\left[h_{1} \circ f^{-1}\right]=\left[h \circ f^{-1}\right]$, that is, $\left[p_{1}\right]=[p]$. 
Finally, suppose $\left[f_{1}\right]=[f]$, and let $p_{1}=h \circ f_{1}^{-1}$. There exists $\sigma \in G_{r_{1}} \times \cdots \times G_{r_{u}}$ such that $f_{1}=\sigma \circ f$. Because of Remark 4.4 and Condition 2(b)(i) of Definition 4.5, we know that there exists $\tau \in G_{k_{1}} \times \cdots \times G_{k_{i}}$ such that $p \circ \sigma^{-1}=\tau \circ p$. Thus $p_{1}=h \circ f_{1}^{-1}=h \circ f^{-1} \circ \sigma^{-1}=p \circ \sigma^{-1}=\tau \circ p$, and therefore $[p]=\left[p_{1}\right]$.

\section{TeChNicAl Lemmas ON MORPhisms in $\mathcal{F}_{\infty} \mathcal{C}$}

In this section, we establish technical lemmas about decompositions of morphisms in $\mathcal{F}_{\infty} \mathcal{C}$ that are used in later sections of the paper. The category $\mathcal{C}$ is assumed to be a category of admissible isomorphisms of an underlying monogenic category $\mathcal{B}$, as in Section 4 . The first decomposition result of this section is Lemma 5.4, which says that a morphism in $\mathcal{F}_{\infty} \mathcal{C}$ that looks like the coproduct of two morphisms is in fact a coproduct of two morphisms in $\mathcal{F}_{\infty} \mathcal{C}$. The main results are Lemma 5.6, which gives a particular type of coproduct decomposition of a morphism in $\mathcal{F}_{\infty} \mathcal{C}$, and Lemma 5.9 , which is a weak naturality result for the construction of Lemma 5.6.

We need an assumption on the coproduct in $\mathcal{B}$, and a resulting lemma and corollary that will be used in the proofs of the decomposition lemmas that comprise the rest of the section. Since $\mathcal{B}$ is assumed to be pointed, we have a projection $\pi_{A}: A \oplus B \rightarrow A \oplus 0=A$ with the property that the composite $A \hookrightarrow A \oplus B \rightarrow A$ is the identity, and that the composite $B \hookrightarrow A \oplus B \rightarrow A$ is the null map. Similarly, there is a projection $\pi_{B}: A \oplus B \rightarrow B$.

We assume the following axiom on the coproduct in $\mathcal{B}$ for the remainder of the paper.

Axiom 5.1 (Injectivity Axiom). The pointed category $\mathcal{B}$ has the property that if $f, f^{\prime}: A \rightarrow C \oplus D$ satisfy $\pi_{C} \circ f=\pi_{C} \circ f^{\prime}$ and $\pi_{D} \circ f=\pi_{D} \circ f^{\prime}$, then $f=f^{\prime}$.

The axiom means, essentially, that the canonical map $B \oplus C \rightarrow B \times C$ from the coproduct in $\mathcal{B}$ to the product is an injection (provided these notions make sense in $\mathcal{B}$ ). The axiom is satisfied by the categories of finite pointed sets, finitelygenerated vector spaces, and finitely-generated free modules. However, the category of finitely-generated free groups does not satisfy the axiom, and therefore nothing we do from here on has any relevance to this example. In fact, the reason we mentioned the example of free groups is to show that not every reasonable-looking monogenic category satisfies our additional axioms.

The most important immediate consequence of the Injectivity Axiom is the following lemma.

Lemma 5.2. Let $f: A \oplus B \rightarrow C \oplus D$ have the property that $A \hookrightarrow A \oplus B \rightarrow$ $C \oplus D \rightarrow D$ and $B \hookrightarrow A \oplus B \rightarrow C \oplus D \rightarrow C$ are null. Then $f$ is the coproduct of two maps $f^{\prime}: A \rightarrow C$ and $f^{\prime \prime}: B \rightarrow D$.

Proof. Let $f^{\prime}$ be the composite $A \hookrightarrow A \oplus B \rightarrow C \oplus D \rightarrow C$ and let $f^{\prime \prime}$ be the composite $B \hookrightarrow A \oplus B \rightarrow C \oplus D \rightarrow D$. To compare $f$ with $f^{\prime} \oplus f^{\prime \prime}$, we first compose with projection to $C$,

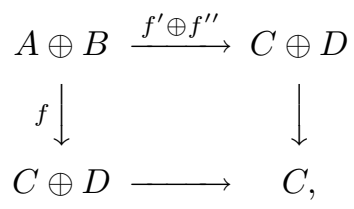


and note that both ways around the square are $f^{\prime}$ on $A$ and null on $B$. Likewise, when we replace the lower right corner with $D$, we find that both ways around the square are null on $A$ and $f^{\prime \prime}$ on $B$. This concludes the proof, by the Injectivity Axiom.

Corollary 5.3. A morphism $f: A \rightarrow C \oplus D$ has the property that the composite $A \rightarrow C \oplus D \rightarrow D$ is null if and only if $f$ factors through the inclusion $C \hookrightarrow C \oplus D$.

The first decomposition lemma of this section, Lemma 5.4, is actually in service of Lemma 5.9, and has to do with recognizing a morphism of $\mathcal{F}_{\infty} \mathcal{C}$ as a coproduct of two other morphisms in $\mathcal{F}_{\infty} \mathcal{C}$. Here is the setting. Let $A=\left[a ; n_{1}, \ldots, n_{t}, k_{1}, \ldots, k_{i}\right]$ and $B=\left[b ; r_{1}, \ldots, r_{u}, l_{1}, \ldots, l_{j}\right]$ be objects of $\mathcal{F}_{\infty} \mathcal{C}$, and let $[f]: A \rightarrow B$ be a morphism in $\mathcal{F}_{\infty} \mathcal{C}$. Let $n_{\bullet}$ denote the sequence $n_{1}, \ldots, n_{t}$, and define $k_{\bullet}, r_{\bullet}$, and l• similarly. Define $g^{n_{\bullet}}=g^{n_{1}} \oplus \cdots \oplus g^{n_{t}}$, and define $g^{k_{\bullet}}, g^{r_{\bullet}}$, and $g^{l_{\bullet}}$ similarly. Suppose given integers $n, k, r$, and $l$ such that $a=n+k$ and $b=r+l$, and suppose that a representative $f$ of $[f]$ factors as a coproduct in the following sense: there are $\mathcal{C}$-admissible isomorphisms $f_{1}, f_{2}, h$, and $h^{\prime}$ in $\mathcal{B}$ such that the following diagram commutes,

$$
\begin{array}{ccc}
g^{a} \oplus g^{n} \oplus g^{k} & f & g^{b} \oplus g^{r} \oplus g^{l} \\
h \downarrow \cong & h^{\prime} \downarrow \cong \\
\left(g^{n} \oplus g^{n}\right) \oplus\left(g^{k} \oplus g^{k}\right) & \stackrel{f_{1} \oplus f_{2}}{\longrightarrow}\left(g^{r} \oplus g^{r}\right) \oplus\left(g^{l} \oplus g^{l}\right),
\end{array}
$$

where the vertical arrows use $\mathcal{C}$-admissible isomorphisms $h: g^{a} \rightarrow g^{n} \oplus g^{k}$ and $h^{\prime}: g^{b} \rightarrow g^{r} \oplus g^{l}$, together with with the identity on the $g^{n}, g^{k_{\bullet}}, g^{r} \bullet$, and $g^{l}$ and the commutativity isomorphisms of $\mathcal{B}$.

Lemma 5.4. The maps $f_{1}$ and $f_{2}$ satisfy the conditions of Definition 4.5 to represent morphisms in $\mathcal{F}_{\infty} \mathcal{C}$, namely $\left[f_{1}\right]:\left[n ; n_{\bullet}\right] \rightarrow\left[r ; r_{\bullet}\right]$ and $\left[f_{2}\right]:\left[k ; k_{\bullet}\right] \rightarrow\left[l ; l_{\bullet}\right]$, respectively.

Proof. We need to check Conditions 2(b)(i) and 2(b)(ii) in Definition 4.5 in order to know that $f_{1}$ and $f_{2}$ actually do represent morphisms in $\mathcal{F}_{\infty} \mathcal{C}$. We concentrate our attention on $f_{1}$, the proof for $f_{2}$ being exactly analogous.

Condition 2(b)(i) is essentially inherited from $f$ itself. We know that $f_{1} \oplus f_{2}$ restricted to $g^{n}$ is null into $g^{l} \bullet$, and thus $f$ also has this property. Likewise, $f$ restricted to $g^{k}$ is null into $g^{r} \bullet$. It follows from Condition 2(b)(i) for $f$ that the set map $\alpha_{f}: \underline{t+i} \rightarrow \underline{u+j}$ is in fact the coproduct of two maps $\alpha_{f_{1}}: \underline{t} \rightarrow \underline{u}$ and $\alpha_{f_{2}}: \underline{i} \rightarrow \underline{j}$. Likewise, the necessary factoring property in Condition 2(b)(i) for $f_{1}$ follows from the corresponding factoring property for $f$.

Now for the real issue. We need to produce a map $\theta_{f_{1}}$ that satisfies Condition $2(\mathrm{~b})$ (ii) for $f_{1}$. Suppose that we are given a representative isomorphism

$$
\theta_{f}: g^{a} \rightarrow g^{b} \oplus g^{e\left(r_{1}\right)} \oplus \cdots \oplus g^{e\left(r_{u}\right)} \oplus g^{e\left(l_{1}\right)} \oplus \cdots \oplus g^{e\left(l_{j}\right)}
$$


Consider the following sequence of morphisms, where all of the maps are either cofibrations or $\mathcal{C}$-admissible isomorphisms.

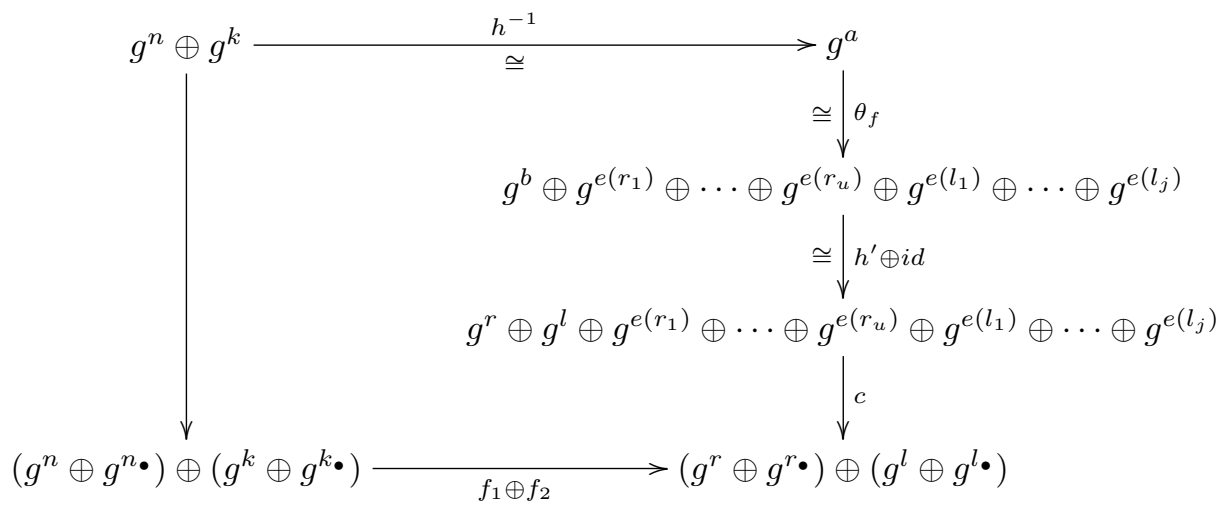

(The map $c$ uses the identity on $g^{r}$ and $g^{l}$, cofibrations $g^{e\left(r_{1}\right)} \hookrightarrow g^{r_{1}}$, etc., and the commutativity isomorphisms of the coproduct in the category $\mathcal{B}$, while the left vertical inclusion is the standard inclusion.) Following $g^{n}$ counterclockwise from the top left to the bottom right shows that $g^{n}$ is null into $g^{l} \oplus g^{l} \bullet$, and since $c$ is the coproduct of cofibrations and isomorphisms, this means that $g^{n}$ followed clockwise from top left to bottom right is actually null into $g^{l} \oplus g^{e\left(l_{1}\right)} \oplus \cdots \oplus g^{e\left(l_{j}\right)}$ in the domain of $c$ as well. Thus $f_{1}$ restricted to $g^{n}$ factors as

$$
g^{n} \rightarrow g^{r} \oplus g^{e\left(r_{1}\right)} \oplus \cdots \oplus g^{e\left(r_{u}\right)} \hookrightarrow g^{r} \oplus g^{r} \bullet
$$

and we define the first of these maps to be $\theta_{f_{1}}$. Following parallel reasoning for $f_{2}$, we find that $f_{2}$ restricted to $g^{k}$ factors as

$$
g^{k} \rightarrow g^{l} \oplus g^{e\left(l_{1}\right)} \oplus \cdots \oplus g^{e\left(l_{u}\right)} \hookrightarrow g^{l} \oplus g^{l_{\bullet}},
$$

and we define the first of these maps to be $\theta_{f_{2}}$. Finally, when restricted to $g^{n} \oplus g^{k}$, the composite $\left(h^{\prime} \oplus i d\right) \circ \theta_{f} \circ h^{-1}$ is a $\mathcal{C}$-admissible isomorphism. Thus by Lemma 5.2 and Condition (4) of Definition 4.2, the maps $\theta_{f_{1}}$ and $\theta_{f_{2}}$ are both $\mathcal{C}$-admissible isomorphisms, as required, which finishes the proof.

Our main goal for this section is a construction to decompose a morphism of $\mathcal{F}_{\infty} \mathcal{C}$ into a coproduct of two other morphisms once given a decomposition of the target. In fact, we do not establish this in complete generality, for the sake of ease of notation, but only in the special case that we will need in later sections, namely the case where one summand ( $R_{B}$ below) has no "free" component.

Let $[f]: N=\left[n ; n_{1}, \ldots, n_{t}\right] \rightarrow R=\left[r ; r_{1}, \ldots, r_{u}\right]$ be a morphism in $\mathcal{F}_{\infty} \mathcal{C}$ with an associated isomorphism $\theta_{f}: g^{n} \rightarrow g^{r} \oplus\left(\oplus_{j=1}^{j=u} g^{e_{j}}\right)$. Let $A \cup B$ be a partition of the set $\underline{u}$, and let $e=\sum_{j \in B} e_{j}$. If $j_{1}<\cdots<j_{u^{\prime}}$ are the elements of $A$, let $r_{A}$ denote the sequence $r_{j_{1}}, \ldots, r_{j_{u^{\prime}}}$, and define $g^{r_{A}}=g^{r_{j_{1}}} \oplus \cdots \oplus g^{r_{j_{u^{\prime}}}}$. Define $r_{B}$ and $g^{r_{B}}$ similarly, and define $R_{A}=\left[r ; r_{A}\right]$ and $R_{B}=\left[0 ; r_{B}\right]$. We make similar definitions for $n_{\alpha_{f}^{-1}(A)}, n_{\alpha_{f}^{-1}(B)}, g^{n_{\alpha_{f}^{-1}(A)}}$, and $g^{n_{\alpha_{f}^{-1}(B)}}$. Let $N_{A}=\left[n-e ; n_{\alpha_{f}^{-1}(A)}\right]$ and $N_{B}=\left[e ; n_{\alpha_{f}^{-1}(B)}\right]$.

The isomorphism $\theta_{f}$ gives us a commuting diagram as follows. 


\section{Diagram 5.5.}

$$
\begin{aligned}
& g^{n} \oplus g^{n_{1}} \oplus \cdots \oplus g^{n_{t}} \\
& \cong \theta_{f} \oplus i d \\
& {[\underbrace{g^{r} \oplus\left(\oplus_{j \in A} g^{e_{j}}\right)}_{g^{n-e}} \oplus g^{n_{\alpha_{f}^{-1}(A)}}] \oplus[\underbrace{\left(\oplus_{j \in B} g^{e_{j}}\right)}_{g^{e}} \oplus g^{n_{\alpha_{f}^{-1}(B)}}] \stackrel{d_{f}}{\longrightarrow} \quad\left(g^{r} \oplus g^{r_{A}}\right) \oplus g^{r_{B}}}
\end{aligned}
$$

Here the left vertical arrow uses $\theta_{f}$ and the commutativity isomorphisms for the coproduct in the category $\mathcal{B}$, the right vertical arrow uses only the commutativity isomorphisms in $\mathcal{B}$, and $d_{f}$ is defined to make the diagram commute.

Lemma 5.6. The isomorphism $d_{f}$ in Diagram 5.5 splits as the coproduct of two isomorphisms $f_{A}: g^{n-e} \oplus g^{n_{\alpha_{f}^{-1}(A)}} \rightarrow g^{r} \oplus g^{r_{A}}$ and $f_{B}: g^{e} \oplus g^{n_{\alpha_{f}^{-1}(B)}} \rightarrow g^{r_{B}}$ that satisfy the conditions of Definition 4.5 to represent, respectively, morphisms $\left[f_{A}\right]: N_{A} \rightarrow R_{A}$ and $\left[f_{B}\right]: N_{B} \rightarrow R_{B}$ in $\mathcal{F}_{\infty} \mathcal{C}$. Further, $\left[f_{A}\right]$ and $\left[f_{B}\right]$ fit into a commutative diagram

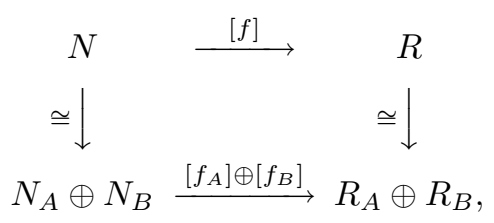

where the vertical isomorphisms are represented by the vertical maps in Diagram 5.5.

Proof. The restriction of $d_{f}$ to $g^{n-e} \oplus g^{n_{\alpha}-1(A)}$ is null into the components $g^{r_{B}}$, and likewise $d_{f}$ restricted to $g^{e} \oplus g^{n_{\alpha_{f}^{-1}(B)}}$ is null into the components $g^{r_{A}}$. Thus by Lemma 5.2, we find that $d_{f}$ is the coproduct of morphisms $f_{A}: g^{n-e} \oplus g^{n_{\alpha}-1(A)} \rightarrow$ $g^{r_{A}}$ and $f_{B}: g^{e} \oplus g^{n_{\alpha-1}(B)} \rightarrow g^{r_{B}}$. Since $d_{f}$ is a $\mathcal{C}$-admissible isomorphism, Condition (4) of Definition 4.2 tells us that each of $f_{A}$ and $f_{B}$ are $\mathcal{C}$-admissible isomorphisms. By construction, they satisfy the conditions of Definition 4.5, which finishes the proof of the lemma.

It is important to keep in mind in the discussion above that the choice of $\theta_{f}$ is not unique. In fact, the difficulty in using the construction of Lemma 5.6 stems from the fact that the isomorphism $N \cong N_{A} \oplus N_{B}$ in Lemma 5.6 is not canonical, but depends in an essential way on the particular choice used for $\theta_{f}$. The existence of choices means that the decomposition will not be natural in a strict sense. Fortunately, for everything that we need to do, we only need to know that compatible choices can be made so that appropriate diagrams commute. To be more precise, suppose that we have a commutative diagram in $\mathcal{F}_{\infty} \mathcal{C}$,

$$
\begin{gathered}
N^{\prime}=\left[n^{\prime} ; n_{1}^{\prime}, \ldots, n_{t^{\prime}}^{\prime}\right] \stackrel{\left[f^{\prime}\right]}{\longrightarrow} R^{\prime}=\left[r^{\prime} ; r_{1}^{\prime}, \ldots, r_{u^{\prime}}^{\prime}\right] \\
{[p] \mid} \\
N=\left[n ; n_{1}, \ldots, n_{t}\right] \stackrel{[f]}{\longrightarrow} R=\left[r ; r_{1}, \ldots, r_{u}\right],
\end{gathered}
$$

and suppose that $A \cup B$ is a partition of $\underline{u}$. Let $A^{\prime}=\alpha_{q}^{-1}(A)$ and let $B^{\prime}=\alpha_{q}^{-1}(B)$. We would like the construction of Lemma 5.6, applied to $[f]$ and to $\left[f^{\prime}\right]$, to yield a commutative diagram as follows. 


\section{Diagram 5.7.}

$$
\begin{aligned}
& N^{\prime} \stackrel{\cong}{\longrightarrow} N_{A^{\prime}}^{\prime} \oplus N_{B^{\prime}}^{\prime} \stackrel{\left[f_{A^{\prime}}^{\prime}\right] \oplus\left[f_{B^{\prime}}^{\prime}\right]}{\longrightarrow} R_{A^{\prime}}^{\prime} \oplus R_{B^{\prime}}^{\prime} \stackrel{\cong}{\longrightarrow} \\
& {[p] \downarrow \quad\left[p_{A}\right] \oplus\left[p_{B}\right] \downarrow \quad\left[q_{A}\right] \oplus\left[q_{B}\right] \downarrow \quad[q] \downarrow} \\
& N \stackrel{\cong}{\longrightarrow} N_{A} \oplus N_{B} \stackrel{\left[f_{A} \oplus f_{B}\right]}{\longrightarrow} R_{A} \oplus R_{B} \stackrel{\cong}{\longrightarrow} .
\end{aligned}
$$

(Here the composite across the top row should be $\left[f^{\prime}\right]$ and the composite across the bottom row should be $[f]$.)

However, that turns out to be false, for essentially trivial reasons, unless we make the additional assumption that $e_{[q]}\left(r_{j}\right)=0$ for $j \in B$. Hence we will make this assumption as a hypothesis in the lemma below. The construction of Lemma 5.6 gives us a commuting diagram as follows, where the top square comes from applying the lemma to $\left[f^{\prime}\right]$, and the bottom square comes from applying it to $[f]$.

\section{Diagram 5.8.}

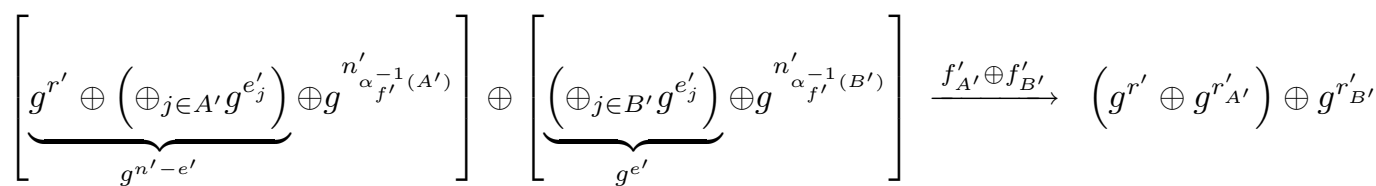

$$
\begin{aligned}
& c_{\theta_{f^{\prime}}} \uparrow \cong \\
& g^{n^{\prime}} \oplus g^{n_{1}^{\prime}} \oplus \cdots \oplus g^{n_{t^{\prime}}^{\prime}} \\
& p \downarrow \\
& g^{n} \oplus g^{n_{1}} \oplus \cdots \oplus g^{n_{t}} \\
& c_{\theta_{f}} \downarrow \\
& {[\underbrace{g^{r} \oplus\left(\oplus_{j \in A} g^{e_{j}}\right)}_{g^{n-e}} \oplus g^{n_{\alpha_{f}^{-1}(A)}}] \oplus[\underbrace{\left(\oplus_{j \in B} g^{e_{j}}\right)}_{g^{e}} \oplus g^{n_{\alpha_{f}^{-1}(B)}}] \quad \stackrel{f_{A} \oplus f_{B}}{\longrightarrow} \quad\left(g^{r} \oplus g^{r_{A}}\right) \oplus g^{r_{B}}}
\end{aligned}
$$

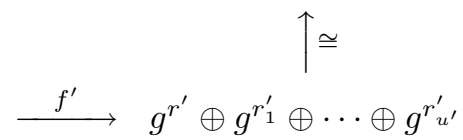

The naturality result we need for the use of Lemma 5.6 is that the corners of this diagram form a square that is the coproduct of two squares in $\mathcal{F}_{\infty} \mathcal{C}$.

Lemma 5.9. Assuming that $e_{[q]}\left(r_{j}\right)=0$ for $j \in B$, the map $c_{\theta_{f}} \circ p \circ c_{\theta_{f^{\prime}}}^{-1}$ is the coproduct of two $\mathcal{C}$-admissible isomorphisms in $\mathcal{B}$,

$$
\begin{aligned}
& p_{A}:\left[g^{n^{\prime}-e^{\prime}} \oplus g^{n_{\alpha_{f^{\prime}}^{\prime}\left(A^{\prime}\right)}^{-1}}\right] \rightarrow\left[g^{n-e} \oplus g^{n_{\alpha_{f}^{-1}(A)}}\right] \\
& p_{B}:\left[g^{e^{\prime}} \oplus g^{n_{\alpha_{f^{\prime}}^{\prime}\left(B^{\prime}\right)}^{-1}}\right] \rightarrow\left[g^{e} \oplus g^{n_{\alpha_{f}^{-1}(B)}^{-1}}\right],
\end{aligned}
$$

and $p_{A}$ and $p_{B}$ represent morphisms in $\mathcal{F}_{\infty} \mathcal{C}$,

$$
\begin{aligned}
& {\left[p_{A}\right]:\left[n^{\prime}-e^{\prime} ; n_{\alpha_{f^{\prime}}^{\prime}\left(A^{\prime}\right)}^{\prime}\right] \rightarrow\left[n-e ; n_{\alpha_{f}^{-1}(A)}\right]} \\
& {\left[p_{B}\right]:\left[e^{\prime} ; n_{\alpha_{f^{\prime}}^{\prime}\left(B^{\prime}\right)}^{\prime-1}\right] \rightarrow\left[e ; n_{\alpha_{f}^{-1}(B)}\right] .}
\end{aligned}
$$

The morphism $\left[p_{A}\right] \oplus\left[p_{B}\right]$ makes Diagram 5.7 commute. 
Proof. Combining the assumption that $\alpha: \underline{u^{\prime}} \rightarrow \underline{u}$ is a coproduct of two maps $\alpha_{q_{B}}: B^{\prime} \rightarrow B$ and $\alpha_{q_{A}}: A^{\prime} \rightarrow A$ with the assumption that $q$ is a $\mathcal{C}$-admissible isomorphism satisfying the conditions of Definition 4.5, together with the assumption that $e_{[q]}\left(r_{j}\right)=0$ for $j \in B$, we know that the composition from the top right corner of Diagram 5.8 to the bottom right corner is the coproduct of a $\mathcal{C}$-admissible isomorphism $q_{B}: g^{r_{B^{\prime}}^{\prime}} \rightarrow g^{r_{B}}$ with a $\mathcal{C}$-admissible isomorphism $q_{A}: g^{r^{\prime}} \oplus g^{r_{A^{\prime}}^{\prime}} \rightarrow g^{r} \oplus g^{r_{A}}$. We can abstract from Diagram 5.8 the following.

\section{Diagram 5.10.}

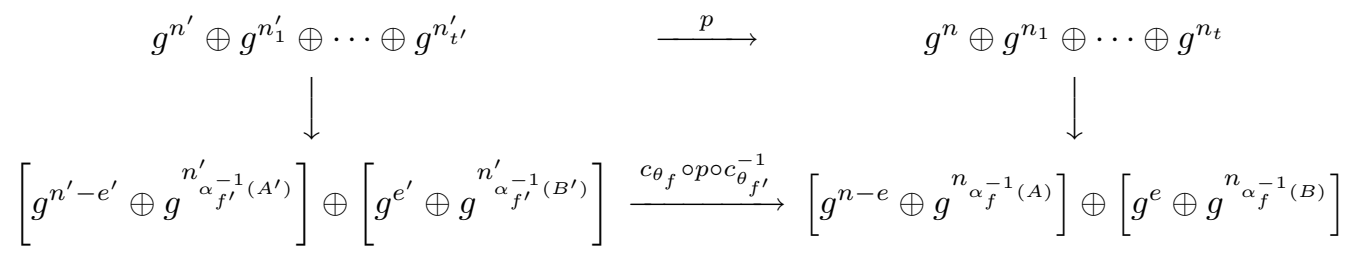

The bottom arrow is the same as going around Diagram 5.8 counterclockwise from bottom left to top left, and therefore is the coproduct of two $\mathcal{C}$-admissible isomorphisms $p_{A}$ and $p_{B}$ as stated in the lemma, and $p_{A}$ and $p_{B}$ represent morphisms in $\mathcal{F}_{\infty} \mathcal{C}$ by Lemma 5.4 .

\section{FACtoring MORPhisms in $\mathcal{F}_{m} \mathcal{C}$}

In this section and the next, $\mathcal{B}$ is a monogenic category satisfying Axiom 5.1, and $\mathcal{C}$ is a subcategory of admissible isomorphisms. Recall that $\mathcal{F}_{m} \mathcal{C}$ is the full subcategory of $\mathcal{F}_{\infty} \mathcal{C}$ whose objects are of the form $\left[n ; n_{1}, \ldots, n_{t}\right]$ where $n_{i} \leq m$ for all $i$. Our goal in this middle part of the paper is Theorem 7.1, which establishes that $\mathcal{F}_{m} \mathcal{C}$ and the category $\mathcal{K}_{m} \mathcal{C}$ defined in Construction 3.8 are linked by a chain of monoidal functors that induce homotopy equivalences on classifying spaces. By definition, $\mathcal{F}_{0} \mathcal{C} \cong \mathcal{C}=\mathcal{K}_{0} \mathcal{C}$, and we will show in Section 7 that $\mathcal{F}_{m} \mathcal{C}$ is related to $\mathcal{F}_{m-1} \mathcal{C}$ in the same way that $\mathcal{K}_{m} \mathcal{C}$ is related to $\mathcal{K}_{m-1} \mathcal{C}$, namely by a homotopy pushout. The desired conclusion will follow by induction.

This section is entirely in service of Section 7 and establishes two technical results. We begin with a definition involving two types of morphisms in $\mathcal{F}_{m} \mathcal{C}$ that, it turns out, need to be tweezed apart in order to analyze $B \mathcal{F}_{m} \mathcal{C}$. We then establish Lemma 6.2, a technical tool about factoring arbitrary morphisms in $\mathcal{F}_{m} \mathcal{C}$ into a composite of these two kinds of morphisms. Finally, we apply Lemma 6.2 to prove Proposition 6.3, which shows the equivalence of the nerves of two categories of chains in $\mathcal{F}_{m} \mathcal{C}$. These results will be used in Section 7 .

\section{Definition 6.1.}

(1) We say that a morphism $\left[n ; n_{1}, \ldots, n_{t}\right] \rightarrow\left[r ; r_{1}, \ldots, r_{u}\right]$ in $\mathcal{F}_{m} \mathcal{C}$ creates no components of augmentation $m$ if the number of values equal to $m$ in the lists $n_{1}, \ldots, n_{t}$ and $r_{1}, \ldots, r_{u}$ is the same.

(2) We say that a morphism $\left[n ; n_{1}, \ldots, n_{t}\right] \rightarrow\left[r ; r_{1}, \ldots, r_{u}\right]$ in $\mathcal{F}_{m} \mathcal{C}$ creates only components of augmentation $m$ if the associated map of sets $\alpha: \underline{t} \rightarrow \underline{u}$ has the property that every element of $\left\{j \in \underline{u} \mid r_{j}<m\right\}$ has a single preimage under $\alpha$, and $n_{\alpha^{-1}(j)}=r_{j}$.

(3) We write $\mathcal{F}_{m}^{\prime} \mathcal{C}$ for the subcategory of $\mathcal{F}_{m} \mathcal{C}$ consisting of morphisms that only create components of augmentation $m$. 
Note that $\mathcal{F}_{m}^{\prime} \mathcal{C}$ is not a full subcategory of $\mathcal{F}_{m} \mathcal{C}$, but it is a monoidal subcategory.

Lemma 6.2. Let $N=\left[n ; n_{1}, \ldots, n_{t}\right]$ and $R=\left[r ; r_{1}, \ldots, r_{u}\right]$ be objects in $\mathcal{F}_{m} \mathcal{C}$.

(1) A morphism $N \rightarrow R$ can be factored as a composite $N \rightarrow R^{\prime} \rightarrow R$ in $\mathcal{F}_{m} \mathcal{C}$ such that $N \rightarrow R^{\prime}$ creates only components of augmentation $m$ and $R^{\prime} \rightarrow R$ creates no components of augmentation $m$. The object $R^{\prime}$ is unique up to isomorphism.

(2) Given a commutative diagram

$$
\begin{array}{cc}
N=\left[n ; n_{1}, \ldots, n_{t}\right] \stackrel{[f]}{\longrightarrow} R=\left[r ; r_{1}, \ldots, r_{u}\right] \\
{[p]} \\
K=\left[k ; k_{1}, \ldots, k_{i}\right] \stackrel{[h]}{\longrightarrow} L=\left[l ; l_{1}, \ldots, l_{j}\right]
\end{array}
$$

such that the vertical maps create no components of augmentation $m$, and given factorings $N \rightarrow R^{\prime} \rightarrow R$ and $K \rightarrow L^{\prime} \rightarrow L$ as in $(1)$ of $[f]$ and $[h]$, respectively, there exists a unique morphism $R^{\prime} \rightarrow L^{\prime}$ to expand (6.1) to a commuting ladder

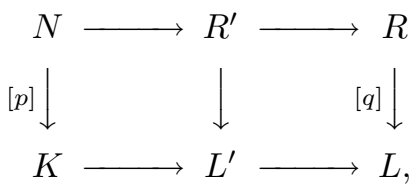

and $R^{\prime} \rightarrow L^{\prime}$ creates no components of augmentation $m$.

Proof. The uniqueness of the object $R^{\prime}$ up to isomorphism is immediate from Definition 6.1. We will use Lemma 5.6 and Lemma 5.9 to construct the factoring and to address the compatibility with commutative diagrams.

Let $B=\left\{s \in \underline{u} \mid r_{s}=m\right\}$, and let $A=\underline{u}-B$. Then using Lemma 5.6 (and expanding its diagram slightly), we have a diagram

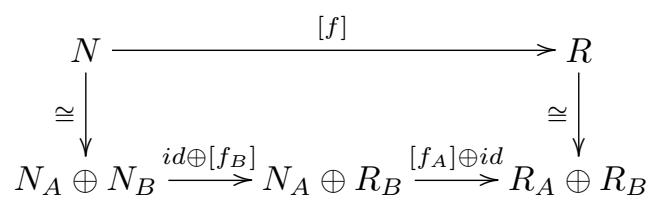

Then by construction, $i d \oplus\left[f_{B}\right]$ creates only components of augmentation $m$, while $\left[f_{A}\right] \oplus i d$ creates no components of augmentation $m$, and we define $R^{\prime}=N_{A} \oplus R_{B}$ and factor $[f]$ by going counterclockwise around the diagram from $N$ to $R$.

Further, suppose given a commutative diagram (6.1) where the vertical morphisms create no components of augmentation $m$. Let $D=\left\{s \in \underline{j} \mid l_{s}=m\right\}$, and let $C=\underline{j}-D$. Because $[q]$ creates no components of augmentation $m$, we know that $\alpha_{q}$ is a one-to-one correspondence from $B$ to $D$, and clearly for $s \in B$ we have $r_{s}=l_{\alpha_{q}(s)}$, since both are $m$ by hypothesis. Thus (6.1) satisfies the conditions of Lemma 5.9, and given fixed isomorphisms $N \cong N_{A} \oplus N_{B}$ and $K \cong K_{C} \oplus K_{D}$, we get morphisms in $\mathcal{F}_{\infty} \mathcal{C}$ between the pieces, namely $\left[p_{A}\right]: N_{A} \rightarrow K_{C}$ and $\left[p_{B}\right]: N_{B} \rightarrow K_{D}$. Then the following diagram establishes the existence of a map 
$R^{\prime} \rightarrow L^{\prime}$ that satisfies the lemma.

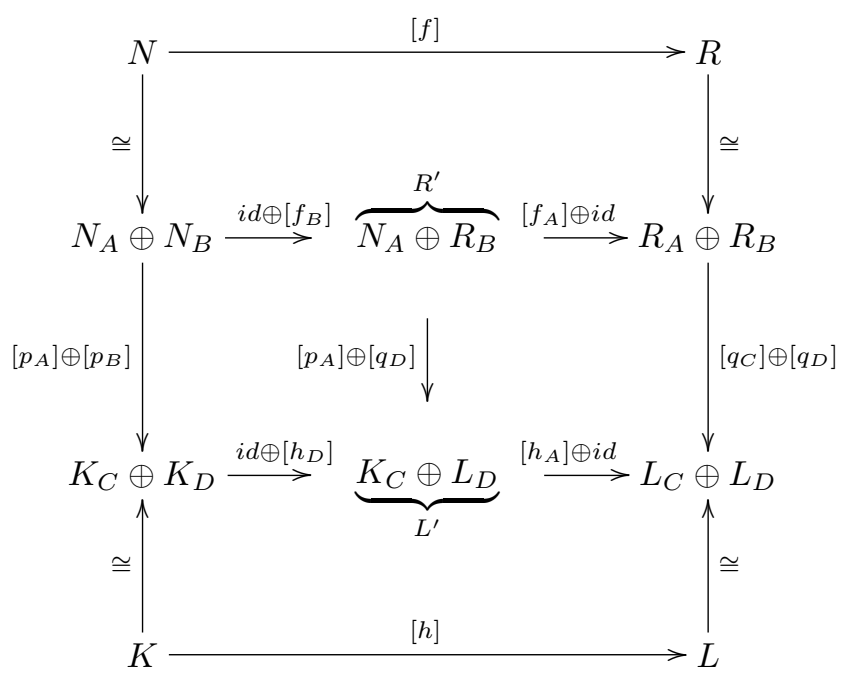

Finally, the uniqueness of $R^{\prime} \rightarrow L^{\prime}$ follows from Lemma 4.12.

The second technical result of this section is in aid of the analysis of a certain bisimplicial set in Section 7. Let $\Xi_{q}\left(\mathcal{F}_{m} \mathcal{C}\right)$ be the category whose objects are $q$ chains $N_{0} \rightarrow \cdots \rightarrow N_{q}$ in $\mathcal{F}_{m} \mathcal{C}$, and whose morphisms are commuting ladders

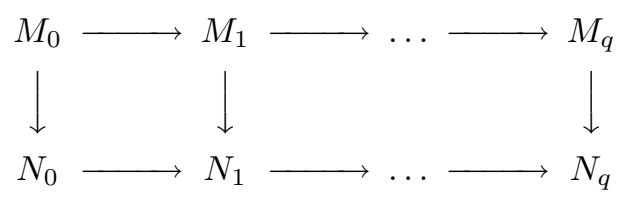

where the vertical maps create no components of augmentation $m$. Let $\Xi_{q}\left(\mathcal{F}_{m}^{\prime} \mathcal{C}\right)$ be the full subcategory of $\Xi_{q}\left(\mathcal{F}_{m} \mathcal{C}\right)$ where the horizontal maps create only components of augmentation $m$. We consider bisimplicial sets in Section 7 whose levels are the nerves of these categories, and we will need the following proposition.

Proposition 6.3. The inclusion functor $\Xi_{q}\left(\mathcal{F}_{m}^{\prime} \mathcal{C}\right) \rightarrow \Xi_{q}\left(\mathcal{F}_{m} \mathcal{C}\right)$ induces a weak equivalence of classifying spaces.

Proof. Fix a chain $N_{0} \rightarrow \cdots \rightarrow N_{q}$, call it $\gamma$, in $\mathcal{F}_{m} \mathcal{C}$; it is sufficient to prove that the over-category $\Xi_{q}\left(\mathcal{F}_{m}^{\prime} \mathcal{C}\right) \downarrow \gamma$ has contractible nerve. To prove this, we construct a chain $\gamma^{\prime}$ that we assert is a terminal object in $\Xi_{q}\left(\mathcal{F}_{m}^{\prime} \mathcal{C}\right) \downarrow \gamma$.

The chain $\gamma^{\prime}$ is defined inductively. We begin by factoring $N_{0} \rightarrow N_{1}$ as in Lemma 6.2(1) to obtain $N_{0} \rightarrow N_{1}^{\prime} \rightarrow N_{1}$, where $N_{0} \rightarrow N_{1}^{\prime}$ creates only components of augmentation $m$, and $N_{1}^{\prime} \rightarrow N_{1}$ creates no components of augmentation $m$. Once $N_{i}^{\prime}$ is defined, we consider the composite $N_{i}^{\prime} \rightarrow N_{i} \rightarrow N_{i+1}$ and define $N_{i+1}^{\prime}$ by factoring $N_{i}^{\prime} \rightarrow N_{i+1}$ as $N_{i}^{\prime} \rightarrow N_{i+1}^{\prime} \rightarrow N_{i+1}$ using Lemma 6.2. Here $N_{i}^{\prime} \rightarrow$ $N_{i+1}^{\prime}$ creates only components of augmentation $m$, and $N_{i+1}^{\prime} \rightarrow N_{i+1}$ creates no components of augmentation $m$. The construction gives us a $q$-chain $\gamma^{\prime}$ in $\Xi_{q}\left(\mathcal{F}_{m}^{\prime} \mathcal{C}\right)$ 
and a commuting ladder mapping $\gamma^{\prime}$ to $\gamma$ :

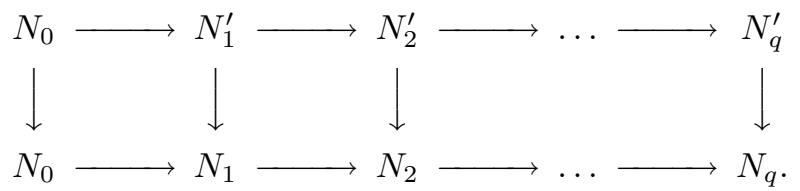

We assert that $(6.2)$ is terminal in $\Xi_{q}\left(\mathcal{F}_{m}^{\prime} \mathcal{C}\right) \downarrow \gamma$. For suppose that

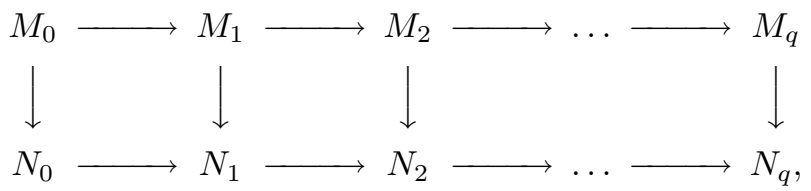

is another object in $\Xi_{q}\left(\mathcal{F}_{m}^{\prime} \mathcal{C}\right) \downarrow \gamma$. Thus the morphisms $M_{i} \rightarrow M_{i+1}$ create only components of augmentation $m$. As a result, if we apply the same construction to $M_{0} \rightarrow M_{1} \rightarrow M_{2} \rightarrow \cdots \rightarrow M_{q}$ that we applied to $\gamma$, we can choose $M_{i}^{\prime}=M_{i}$ for all $i$. Then the ladder

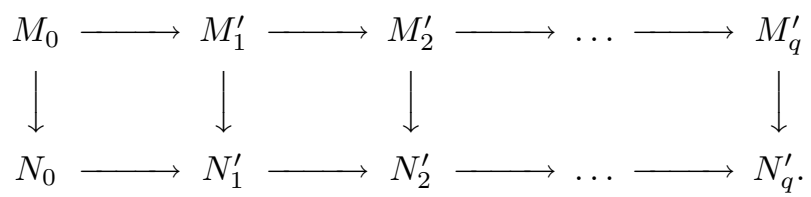

that results from Lemma $6.2(2)$ is actually a ladder

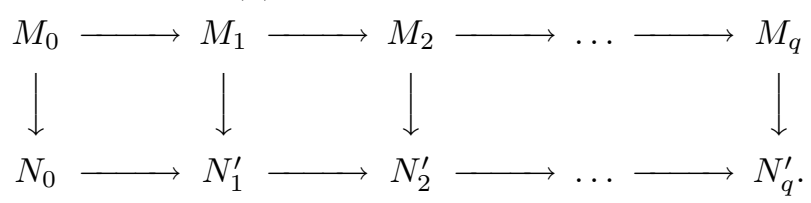

Uniqueness of the vertical maps follows from Lemma 4.12, which finishes the proof.

\section{Linking the COMbinatorial Model to Construction 3.8}

This section builds on the work of Sections 5 and 6 to establish the following theorem, which relates the combinatorial construction of Section 4 to Construction 3.8 .

Theorem 7.1. Let $\mathcal{B}$ be a monogenic category satisfying Axiom 5.1, and let $\mathcal{C}$ be a subcategory of admissible isomorphisms. Then $\mathcal{K}_{m} \mathcal{C}$ and $\mathcal{F}_{m} \mathcal{C}$ are linked by a chain of monoidal functors, each of which induces a homotopy equivalence of classifying spaces. In particular, the associated spectra of $K_{m} \mathcal{C}$ and $\mathcal{F}_{m} \mathcal{C}$ are homotopy equivalent.

Corollary 7.2. Let $\mathcal{C}$ be either the category of finite sets, or the category of finitedimensional vector spaces, or the category of finitely-generated free modules over a ring satisfying the dimension invariance property. Let $A_{0} \rightarrow A_{1} \rightarrow \cdots \rightarrow \mathrm{H} \mathbb{Z}$ be the sequence of spectra (1.2) associated with $\mathcal{C}$. Then there is an equivalence

$$
A_{m} / A_{m-1} \simeq \Sigma^{\infty} \Sigma B \mathcal{F}_{m-1} \mathcal{C}_{m}
$$

Proof of Corollary 7.2. All the categories named in the corollary satisfy the assumptions of Theorem 7.1. It follows from the proof of the theorem that if $\mathcal{C}$ is one of the categories named in the corollary, then $\mathcal{F}_{m} \mathcal{C}$ satisfies, up to homotopy of 
classifying spaces, the same inductive formula as $\mathcal{K}_{m} \mathcal{C}$. In other words, there is a chain of monoidal functors, each one inducing a homotopy equivalence of classifying spaces, connecting $\mathcal{F}_{m} \mathcal{C}$ and $\mathcal{F}_{m-1} \mathcal{C} \otimes_{\text {Free }\left(\mathcal{F}_{m-1} \mathcal{C}_{m}\right)} \mathcal{S}_{m}$. On the level of spectra, this translates to the assertion that there is a homotopy pushout square

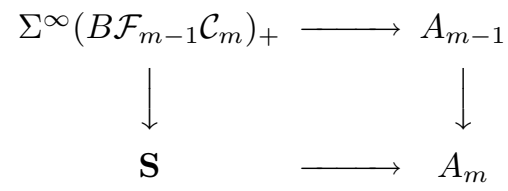

The corollary follows.

The general strategy for proving Theorem 7.1 is to show that $\mathcal{F}_{m} \mathcal{C}$ is related to $\mathcal{F}_{m-1} \mathcal{C}$ in the same way that $\mathcal{K}_{m} \mathcal{C}$ is related to $\mathcal{K}_{m-1} \mathcal{C}$. Specifically, we will show that $B \mathcal{F}_{m} \mathcal{C}$ is equivalent to the geometric realization of the bar construction $\operatorname{Bar}\left(\mathcal{F}_{m-1} \mathcal{C}\right.$, Free $\left.\left(\mathcal{F}_{m-1} C_{m}\right), \mathcal{S}_{m}\right)$. It will then follow by Proposition 2.5 and induction that $B \mathcal{F}_{m} \mathcal{C}$ is equivalent to $B \mathcal{K}_{m} \mathcal{C}$.

We analyze $B \mathcal{F}_{m} \mathcal{C}$ by introducing a bisimplicial set that we can study in the two different simplicial directions. Let $\mathcal{E}^{\prime \prime}\left(\mathcal{F}_{m} \mathcal{C}\right)$ be the bisimplicial set whose $(p, q)$ simplices consist of diagrams in $\mathcal{F}_{m} \mathcal{C}$ of the form

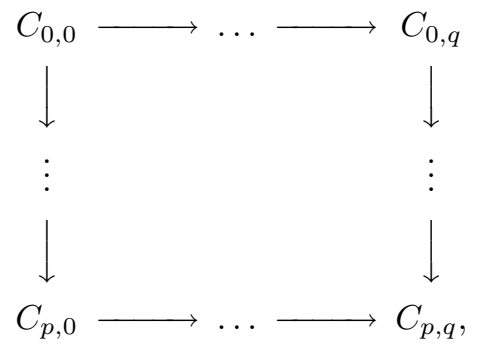

where the vertical maps do not create components of augmentation $m$. Then $\mathcal{E}^{\prime \prime}\left(\mathcal{F}_{m} \mathcal{C}\right)$ is the bisimplicial set of a $\Gamma$-bicategory, and its realization, $\operatorname{diag}\left(\mathcal{E}^{\prime \prime}\left(\mathcal{F}_{m} \mathcal{C}\right)\right)$, is a $\Gamma$-space.

Lemma 7.3. There is a $\Gamma$-equivalence $B \mathcal{F}_{m} \mathcal{C} \simeq \operatorname{diag}\left(\mathcal{E}^{\prime \prime}\left(\mathcal{F}_{m} \mathcal{C}\right)\right)$.

Proof. The proof is exactly as in the proof of Lemma 3.1 of [11]. Namely, for each $p$, there is an equivalence $\mathcal{E}^{\prime \prime}\left(\mathcal{F}_{m} \mathcal{C}\right)_{p, *} \simeq B \mathcal{F}_{m} \mathcal{C}$, and it is in fact a $\Gamma$-equivalence.

We still need to slim down $\mathcal{E}^{\prime \prime}\left(\mathcal{F}_{m} \mathcal{C}\right)$ a little bit. The goal is to tweeze apart the morphisms that create only components of augmentation $m$ from those that create no components of augmentation $m$. Although an arbitrary morphism in $\mathcal{F}_{m} \mathcal{C}$ is neither of these, we proved in Lemma 6.2 that an arbitrary morphism can be factored into a composition of these two types of morphisms. Let $\mathcal{E}^{\prime \prime}\left(\mathcal{F}_{m}^{\prime} \mathcal{C}\right)$ be the sub-bisimplicial set of $\mathcal{E}^{\prime \prime}\left(\mathcal{F}_{m} \mathcal{C}\right)$ consisting of $(p, q)$-simplices where the horizontal morphisms create only new components of augmentation $m$. Recall that $\Xi_{q}\left(\mathcal{F}_{m}^{\prime} \mathcal{C}\right)$ is the category whose objects are $q$-chains in $\mathcal{F}_{m}^{\prime} \mathcal{C}$ (i.e., chains of morphisms that create only components of augmentation $m$ ), with morphisms consisting of commuting ladders where the morphisms between the chains create no new components of augmentation $m$.

Proposition 7.4. $\mathcal{E}^{\prime \prime}\left(\mathcal{F}_{m}^{\prime} \mathcal{C}\right)_{*, q} \hookrightarrow \mathcal{E}^{\prime \prime}\left(\mathcal{F}_{m} \mathcal{C}\right)_{*, q}$ is a weak equivalence. 
Proof. The simplicial sets $\mathcal{E}^{\prime \prime}\left(\mathcal{F}_{m}^{\prime} \mathcal{C}\right)_{*, q}$ and $\mathcal{E}^{\prime \prime}\left(\mathcal{F}_{m} \mathcal{C}\right)_{*, q}$ are, respectively, the nerves of the categories $\Xi_{q}\left(\mathcal{F}_{m}^{\prime} \mathcal{C}\right)$ and $\Xi_{q}\left(\mathcal{F}_{m} \mathcal{C}\right)$. The result follows from Proposition 6.3.

Our task is now to identify the bisimplicial set $\mathcal{E}^{\prime \prime}\left(\mathcal{F}_{m}^{\prime} \mathcal{C}\right)$, whose $(p, q)$-simplices consist of diagrams where the horizontal maps create only components of augmentation $m$ and the vertical maps create no components of augmentation $m$. Let $\mathcal{F}_{m-1} \mathcal{C}_{m}$ denote the full subcategory of $\mathcal{F}_{m-1} \mathcal{C}$ consisting of objects with augmentation exactly $m$. We assert in Proposition 7.8 , below, that $\mathcal{E}^{\prime \prime}\left(\mathcal{F}_{m}^{\prime} \mathcal{C}\right)$ can actually be identified as the bar construction $\operatorname{Bar}\left(\mathcal{F}_{m-1} \mathcal{C}, \operatorname{Free}\left(\mathcal{F}_{m-1} \mathcal{C}_{m}\right), \mathcal{S}_{m}\right)$ up to weak equivalence. Since $\mathcal{E}^{\prime \prime}\left(\mathcal{F}_{m}^{\prime} \mathcal{C}\right)_{*, q}$ is the nerve of $\Xi_{q}\left(\mathcal{F}_{m}^{\prime} \mathcal{C}\right)$, our strategy for proving this is to construct a functor

$$
\xi_{q}: \mathcal{F}_{m-1} \mathcal{C} \times\left(\operatorname{Free}\left(\mathcal{F}_{m-1} \mathcal{C}_{m}\right)\right)^{q} \times \mathcal{S}_{m} \rightarrow \Xi_{q}\left(\mathcal{F}_{m}^{\prime} \mathcal{C}\right)
$$

that gives an equivalence on nerves. Notice that the domain of $\xi_{q}$ is given by the $q$-simplices of the bar construction. The functors $\xi_{\bullet}$ assemble to a map

$$
\operatorname{Bar}\left(\mathcal{F}_{m-1} \mathcal{C}, \operatorname{Free}\left(\mathcal{F}_{m-1} \mathcal{C}_{m}\right), \mathcal{S}_{m}\right) \rightarrow \mathcal{E}^{\prime \prime}\left(\mathcal{F}_{m}^{\prime} \mathcal{C}\right)
$$

which, by Proposition 7.8, induces an equivalence of geometric realizations.

We need two auxiliary functors. For a nonnegative integer $n$, let $\underline{n} m$ indicate the object $[0 ; \underbrace{m, \ldots, m}_{n \text { times }}]$, and note that this gives an embedding of $\overline{\mathcal{S}}_{m}$ as a full subcategory of $\mathcal{F}_{m} \mathcal{C}$. Also note that because $\mathcal{F}_{m-1} \mathcal{C}$ is a permutative category, there is a functor $\mu: \operatorname{Free}\left(\mathcal{F}_{m-1} \mathcal{C}_{m}\right) \rightarrow \mathcal{F}_{m-1} \mathcal{C}$ taking $(\underline{k}, f)$ to the monoidal product of the objects $f(1), \ldots, f(k)$.

To define $\xi_{q}$, we must take an object in $\mathcal{F}_{m-1} \mathcal{C} \times\left(\operatorname{Free}\left(\mathcal{F}_{m-1} \mathcal{C}_{m}\right)\right)^{q} \times \mathcal{S}_{m}$ and obtain a $q$-chain from it. The data supplied are:

- an object $C \in \mathcal{F}_{m-1} \mathcal{C}$,

- a list of objects $\left(\underline{n_{1}}, f_{1}\right), \ldots,\left(n_{q}, f_{q}\right)$ in $\operatorname{Free}\left(\mathcal{F}_{m-1} \mathcal{C}_{m}\right)$, and

- a set $\underline{s}$.

The functor $\xi_{q}$ takes these data to the following $q$-chain:

$$
\begin{aligned}
C \oplus \mu\left(\underline{n_{1}}, f_{1}\right) & \oplus \cdots \oplus \mu\left(\underline{n_{q}}, f_{q}\right) \oplus \underline{s} m \\
& \longrightarrow C \oplus \mu\left(\underline{n_{1}}, f_{1}\right) \oplus \cdots \oplus \mu\left(\underline{n_{q-1}}, f_{q-1}\right) \oplus \underline{n_{q}} m \oplus \underline{s} m \\
& \longrightarrow \cdots \\
& \longrightarrow C \oplus \underline{n_{1}} m \oplus \cdots \oplus \underline{n_{q}} m \oplus \underline{s} m .
\end{aligned}
$$

Remark 7.5. Notice that this $q$-chain is the coproduct of $q+2$ others: the identity $q$-chain on $C$, the identity $q$-chain on $\underline{s} m$, and the $q$-chains formed by taking the morphism $\mu\left(n_{i}, f_{i}\right) \rightarrow \underline{n_{i}} m$ and lengthening it to a $q$-chain by precomposing with $q-i$ identity maps and postcomposing with $i-1$ identity maps. Writing a chain in $\Xi_{q}\left(\mathcal{F}_{m}^{\prime} \mathcal{C}\right)$ in the form just preceding this remark exhibits it as an object in the image of $\xi_{q}$.

\section{Examples 7.6.}

(1) If $q=0$, then $\xi_{0}$ takes an object of $\mathcal{F}_{m-1} \mathcal{C} \times \mathcal{S}_{m}$ and gives a 0 -chain in $\mathcal{F}_{m}^{\prime} \mathcal{C}$. The pair consisting of $\left[n ; n_{1}, \ldots, n_{t}\right]$ and the set $\underline{s}$ goes to the 0 -chain $[n ; n_{1}, \ldots, n_{t}, \underbrace{m, \ldots, m}_{s \text { times }}]$. 
(2) If $q=1$, then $\xi_{1}$ takes an object of $\mathcal{F}_{m-1} \mathcal{C} \times \operatorname{Free}\left(\mathcal{F}_{m-1} \mathcal{C}_{m}\right) \times \mathcal{S}_{m}$ and gives a 1 -chain in $\mathcal{F}_{m}^{\prime} \mathcal{C}$. The given data are $\left[n ; n_{1}, \ldots, n_{t}\right]$ with each $n_{i} \leq m-1$, an object $\left(C_{1}, \ldots, C_{k}\right)$ in $\operatorname{Free}\left(\mathcal{F}_{m-1} \mathcal{C}_{m}\right)$, and a set $\underline{s}$. Note that since each object $C_{i}$ has augmentation $m$, there is a unique morphism $C_{i} \rightarrow[0 ; m]$ in $\mathcal{F}_{m} \mathcal{C}$. This input data gives the 1 -chain

$$
\begin{aligned}
& {\left[n ; n_{1}, \ldots, n_{t}\right] \oplus\left(C_{1} \oplus \cdots \oplus C_{k}\right) \oplus[0 ; \underbrace{m, \ldots, m}_{s \text { times }}] } \\
& \longrightarrow\left[n ; n_{1}, \ldots, n_{t}\right] \oplus(\underbrace{[0 ; m] \oplus \cdots \oplus[0 ; m]}_{k \text { times }}) \oplus[0 ; \underbrace{m, \ldots, m}_{s \text { times }}] .
\end{aligned}
$$

Effectively, we are taking the monoidal product of the identity maps on the outer ends with the unique maps $C_{i} \rightarrow[0 ; m]$ in the middle.

Our goal is to establish that $\xi_{q}$ induces an equivalence of nerves (Proposition 7.8). Our first step is to establish a special case. Let $\iota$ be the restriction of $\xi_{1}$ to Free $\left(\mathcal{F}_{m-1} \mathcal{C}_{m}\right)$. Objects in the image of $\iota$ have the form $[f]: N=\left[n ; n_{1}, \ldots, n_{t}\right] \rightarrow$ $[0 ; m, \ldots, m]$ where $N \in \mathcal{F}_{m-1} \mathcal{C}$, i.e., each $n_{i} \leq m-1$. Let $\mathcal{D}$ denote the full subcategory of $\Xi_{1}\left(\mathcal{F}_{m}^{\prime} \mathcal{C}\right)$ consisting of all objects having this form.

Proposition 7.7. The functor $\iota: \operatorname{Free}\left(\mathcal{F}_{m-1} \mathcal{C}_{m}\right) \rightarrow \mathcal{D}$ induces a weak equivalence of nerves.

Proof. Since $\iota$ is an embedding, it is sufficient to show that the image of $\iota$ contains at least one object in every isomorphism class and is a full subcategory of $\mathcal{D}$. Let $[f]: N=\left[n ; n_{1}, \ldots, n_{t}\right] \rightarrow[0 ; \underbrace{m, \ldots, m}_{u}]$ be a typical object of $\mathcal{D}$, and consider the canonical decomposition $[0 ; m, \ldots, m]=[0 ; m] \oplus \cdots \oplus[0 ; m]$. From Lemma 5.6, we obtain a decomposition

$$
\begin{array}{ccc}
N & \stackrel{[f]}{\longrightarrow} \quad[0 ; m, \ldots, m] \\
\cong & \cong \downarrow \\
N_{1} \oplus \cdots \oplus N_{u} \stackrel{\left[\oplus f_{j}\right]}{\longrightarrow}[0 ; m] \oplus \cdots \oplus[0 ; m]
\end{array}
$$

where $N_{1}, \ldots, N_{u}$ are in $\mathcal{F}_{m-1} \mathcal{C}$. This diagram establishes that $[f]$ is isomorphic to an object in the image of $\iota$, namely the chain in the bottom row.

We must still show the the image of $\iota$ is a full subcategory of $\mathcal{D}$. Suppose that

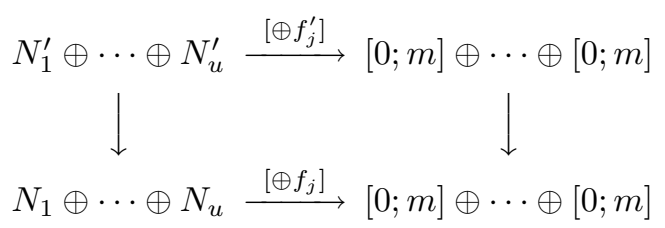

is an arbitrary morphism between objects (the rows) in the image of $\iota$. The right vertical map is necessarily an isomorphism, given by a permutation of the $u$ summands, say $\sigma \in \Sigma_{u}$. Thus by using the given decompositions on the right of the diagram as input to Lemma 5.9, we find that the left vertical map must be the coproduct of morphisms $N_{i}^{\prime} \rightarrow N_{\sigma(i)}$ in $\mathcal{F}_{m-1} \mathcal{C}$, i.e., is a morphism in $\operatorname{Free}\left(\mathcal{F}_{m-1} \mathcal{C}\right)$, as required. 
Proposition 7.8. The functor $\xi_{q}: \mathcal{F}_{m-1} \mathcal{C} \times\left(\operatorname{Free}\left(\mathcal{F}_{m-1} \mathcal{C}_{m}\right)\right)^{q} \times \mathcal{S}_{m} \rightarrow \Xi_{q}\left(\mathcal{F}_{m}^{\prime} \mathcal{C}\right)$ induces a weak equivalence of nerves.

Proof. As in the proof of Proposition 7.7, since $\xi_{q}$ is an embedding, we show that every object in $\Xi_{q}\left(\mathcal{F}_{m}^{\prime} \mathcal{C}\right)$ is isomorphic to one in the image of $\xi_{q}$, and that the image of $\xi_{q}$ is a full subcategory of $\Xi_{q}\left(\mathcal{F}_{m}^{\prime} \mathcal{C}\right)$.

Let us consider a typical chain of $\Xi_{q}\left(\mathcal{F}_{m}^{\prime} \mathcal{C}\right)$,

$$
N_{0} \stackrel{f_{1}}{\longrightarrow} N_{1} \stackrel{f_{2}}{\longrightarrow} \ldots \stackrel{f_{q}}{\longrightarrow} N_{q} .
$$

Thus we are assuming that the maps $f_{1}, \ldots, f_{q}$ create only components of augmentation $m$. We write $N_{i}=\left[n_{i} ; n_{i, 1}, \ldots, n_{i, t_{i}}\right]$. Our strategy is to decompose $N_{q}$ as a coproduct by grouping $n_{q, 1}, \ldots, n_{q, t_{q}}$ into blocks $T_{-1}, T_{0}, \ldots, T_{q}$, and then to use Lemma 5.6 to decompose (7.1) as a coproduct of chains. This will show that (7.1) is isomorphic to a chain in the image of $\xi_{q}$ by Remark 7.5.

To determine the blocks, we group all the elements of $n_{q, 1}, \ldots, n_{q, t_{q}}$ that are less than $m$ together into a block $T_{-1}$. The remaining blocks $T_{0}, \ldots, T_{q}$ hold all the elements $n_{q, j}$ such that $n_{q, j}=m$. To determine which block an element $n_{q, j}$ goes into, we look back in the chain to see how many "preimages" of $n_{q, j}$ are also $m$. (Since we are in $\mathcal{F}_{m} \mathcal{C}$, once $n_{i, j}=m$ we also know its "image" $n_{i+1, \alpha_{f_{i+1}}(j)}=m$, because the image value cannot decrease but also cannot be more than $m$.) To be precise, we partition $\underline{t_{q}}$ as follows:

$$
\begin{aligned}
T_{-1} & =\left\{j \in \underline{t_{q}} \mid n_{q_{j}}<m\right\} \\
T_{0} & =\left\{j \in \underline{t_{q}} \mid \exists k \in \underline{t_{0}} \text { s.t. } n_{0, k}=m \text { and }\left(\alpha_{f_{q}} \ldots \alpha_{f_{1}}\right)(k)=j\right\} \\
T_{1} & =\left\{j \in \underline{t_{q}}-T_{0} \mid \exists k \in \underline{t_{1}} \text { s.t. } n_{1, k}=m \text { and }\left(\alpha_{f_{q}} \ldots \alpha_{f_{2}}\right)(k)=j\right\} \\
& \vdots \\
T_{q-1} & =\left\{j \in \underline{t_{q}}-\left(T_{0} \cup \cdots \cup T_{q-2}\right) \mid \exists k \in \underline{t_{q-1}} \text { s.t. } n_{q-1, k}=m \text { and } \alpha_{f_{q}}(k)=j\right\} \\
T_{q} & =\left\{j \in \underline{t_{q}} \mid n_{q, j}=m, \text { but } \nexists k \in \underline{t_{q-1}} \text { s.t. } n_{q-1, k}=m \text { and } \alpha_{f_{q}}(k)=j\right\}
\end{aligned}
$$

Thus $T_{q}$ labels the components of augmentation $m$ in $N_{q}$ that are newly created by the map $N_{q-1} \rightarrow N_{q}$, while $T_{q-1}$ labels those that were created by the map $N_{q-2} \rightarrow N_{q-1}$, etc. Corresponding to this partition of $t_{q}$, we decompose $N_{q}$ into the coproduct of $q+2$ objects $T_{-1}\left(N_{q}\right), T_{0}\left(N_{q}\right), \ldots, T_{q}\left(\bar{N}_{q}\right)$ namely:

$$
T_{j}\left(N_{q}\right)= \begin{cases}{\left[n_{q} ; n_{T_{-1}}\right]} & j=-1 \\ {\left[0 ; n_{T_{j}}\right]} & 0 \leq j \leq q .\end{cases}
$$

(The notation $n_{T_{j}}$ for a sequence is carried over from Section 5.) Then $N_{q} \cong$ $T_{-1}\left(N_{q}\right) \oplus T_{0}\left(N_{q}\right) \oplus \cdots \oplus T_{q}\left(N_{q}\right)$, and we can apply Lemma 5.6 to decompose $N_{q-1}$ into $N_{q-1} \cong T_{-1}\left(N_{q-1}\right) \oplus T_{0}\left(N_{q-1}\right) \oplus \cdots \oplus T_{q}\left(N_{q-1}\right)$, and the map $f_{q}$ as a coproduct of $q+2$ morphisms $f_{(q, j)}: T_{j}\left(N_{q-1}\right) \rightarrow T_{j}\left(N_{q}\right)$. Note that the summands $T_{j}\left(N_{q-1}\right)$ are defined by the decomposition of $N_{q}$ and the map $f_{q}$, rather than by conditions like those that define $T_{j}\left(N_{q}\right)$, though in fact a similar list of conditions holds.

We make one observation about the maps $f_{(q, j)}$ before we continue. By hypothesis, $f_{q}: N_{q-1} \rightarrow N_{q}$ creates only components of augmentation $m$, and as a consequence, $f_{(q,-1)}$ is an isomorphism. Further $f_{(q, 0)}, \ldots, f_{(q, q-1)}$ are isomorphisms by construction, each one being an isomorphism of objects of the type $[0 ; m, \ldots, m]$. 
Thus at the possible cost of altering the left vertical isomorphism in the diagram

$$
\begin{array}{ccc}
N_{q-1} & \quad N_{q} \\
\cong \downarrow & \cong \downarrow \\
\oplus_{j=-1}^{q} T_{j}\left(N_{q-1}\right) \stackrel{\oplus f_{(q, j)}}{\longrightarrow} \oplus_{j=-1}^{q} T_{j}\left(N_{q}\right),
\end{array}
$$

we can even take $f_{(q,-1)}, f_{(q, 0)}, \ldots, f_{(q, q-1)}$ to be identity maps. This leaves $f_{(q, q)}$ as the only interesting morphism in the coproduct, having the form of a morphism in the category $\mathcal{D}$ studied in Proposition 7.7.

Our next task is to indicate how this procedure will be iterated to decompose the entire chain $N_{0} \rightarrow N_{1} \rightarrow \cdots \rightarrow N_{q}$ into a coproduct of $q+2$ chains, each of which is easily analyzed. Let $h_{q-1}=f_{q} \circ f_{q-1}$, and decompose $N_{q-2}$ as a coproduct of summands $T_{j}\left(N_{q-2}\right)$ by using $h_{q-1}: N_{q-2} \rightarrow N_{q} \cong \oplus_{j=-1}^{q} T_{j}\left(N_{q}\right)$ and applying Lemma 5.6:

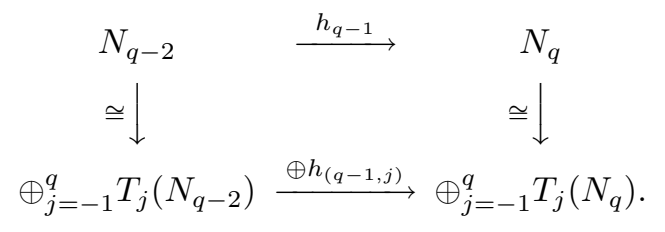

In this case, the same analysis as before allows us to take $h_{(q-1,-1)}, h_{(q-1,0)}, \ldots$, $h_{(q-1, q-2)}$ to be the identity maps, while $h_{(q-1, q-1)}$ and $h_{(q-1, q)}$, like $f_{(q, q)}$, have the form of morphisms in the category $\mathcal{D}$.

There is a commutative diagram

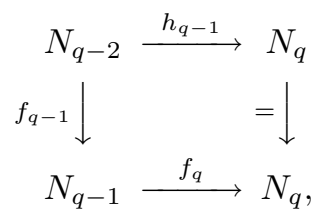

and we have decompositions of all of the terms,

$$
\begin{array}{r}
\oplus_{j=-1}^{q} T_{j}\left(N_{q-2}\right) \stackrel{\oplus h_{(q-1, j)}}{\longrightarrow} \oplus_{j=-1}^{q} T_{j}\left(N_{q}\right) \\
=\downarrow \\
\oplus_{j=-1}^{q} T_{j}\left(N_{q-1}\right) \stackrel{\oplus f_{(q, j)}}{\longrightarrow} \oplus_{j=-1}^{q} T_{j}\left(N_{q}\right) .
\end{array}
$$

By Lemma 5.9, we can fill in to create a commuting square with a morphism $\oplus_{j=-1}^{q} T_{j}\left(N_{q-2}\right) \rightarrow \oplus_{j=-1}^{q} T_{j}\left(N_{q-1}\right)$ that is a coproduct of $q+2$ morphisms, say $f_{(q-1, j)}: T_{j}\left(N_{q-2}\right) \rightarrow T_{j}\left(N_{q-1}\right)$. Further, since we have chosen $h_{(q-1,-1)}, h_{(q-1,0)}$, $\ldots, h_{(q-1, q-2)}$ and $f_{(q,-1)}, f_{(q, 0)}, \ldots, f_{(q, q-2)}$ to be identity maps, we know that $f_{(q-1,-1)}, f_{(q-1,0)}, \ldots, f_{(q-1, q-2)}$ are likewise identity maps. Considering $f_{(q-1, q-1)}$, we see that it has the form of a morphism in $\mathcal{D}$, while $f_{(q-1, q)}$ is an isomorphism (because $N_{q-2} \rightarrow N_{q-1}$ creates only components of augmentation $m$ ), and thus by an adjustment in the isomorphism $N_{q-2} \cong \oplus_{j=-1}^{q} T_{j}\left(N_{q-2}\right)$, we can choose $f_{(q-1, q)}$ to be the identity map. 
After iterating this procedure $q+1$ times, we have a commuting ladder

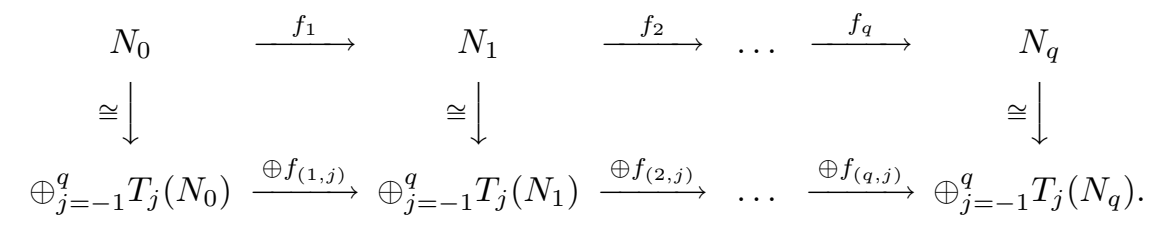

where the top row is the original chain (7.1), and the bottom row is a coproduct of chains

$$
T_{j}\left(N_{0}\right) \stackrel{f_{(1, j)}}{\longrightarrow} T_{j}\left(N_{1}\right) \stackrel{f_{(2, j)}}{\longrightarrow} \ldots \stackrel{f_{(q, j)}}{\longrightarrow} T_{j}\left(N_{q}\right) .
$$

For $1 \leq j \leq q$, we have $T_{j}\left(N_{0}\right)=\cdots=T_{j}\left(N_{j-1}\right)$ and $T_{j}\left(N_{j}\right)=\cdots=T_{j}\left(N_{q}\right)$, and the maps $f_{(1, j)}, \ldots, f_{(j-1, j)}$ and $f_{(j+1, j)}, \ldots, f_{(q, j)}$ are identity maps, while $f_{(j, j)}$ is a morphism in $\mathcal{D}$. For $j=-1,0$, we have $T_{j}\left(N_{0}\right)=\cdots=T_{j}\left(N_{q}\right)$, and all of $f_{(1, j)}$, $\ldots, f_{(q, j)}$ are identity maps.

Recall that our goal was to show that the chain $N_{0} \rightarrow N_{1} \rightarrow \cdots \rightarrow N_{q}$ is isomorphic to a chain in the image of $\xi_{q}: \mathcal{F}_{m-1} \mathcal{C} \times\left(\operatorname{Free}\left(\mathcal{F}_{m-1} \mathcal{C}_{m}\right)\right)^{q} \times \mathcal{S}_{m} \rightarrow \Xi_{q}\left(\mathcal{F}_{m}^{\prime} \mathcal{C}\right)$. From the proof of Proposition 7.7, we know that $T_{j}\left(N_{j-1}\right) \rightarrow T_{j}\left(N_{j}\right)$ is isomorphic to an object in the image of $\iota$, so we replace $T_{j}\left(N_{j-1}\right)$, if necessary, with an object that is actually in the image of $\operatorname{Free}\left(\mathcal{F}_{m-1} \mathcal{C}_{m}\right)$ under $\mu$. Then as a preimage (up to isomorphism) for $N_{0} \rightarrow N_{1} \rightarrow \cdots \rightarrow N_{q}$ in $\mathcal{F}_{m-1} \mathcal{C} \times\left(\operatorname{Free}\left(\mathcal{F}_{m-1} \mathcal{C}_{m}\right)\right)^{q} \times \mathcal{S}_{m}$ under $\xi_{q}$, we take the object that in the $\mathcal{F}_{m-1} \mathcal{C}$-coordinate is $T_{-1}\left(N_{q}\right)$, in the $\left(\text { Free }\left(\mathcal{F}_{m-1} \mathcal{C}_{m}\right)\right)^{q}$-coordinate is $T_{1}\left(N_{q}\right), \ldots, T_{q}\left(N_{q}\right)$, and in the $\mathcal{S}_{m}$-coordinate is $T_{0}\left(N_{q}\right)$. (Here we abuse notation by regarding $T_{0}\left(N_{q}\right)$, which has the form $[0 ; m, \ldots, m]$, as identified with a set of the appropriate cardinality.)

To conclude the proof, we consider a morphism between chains in $\Xi_{q}\left(\mathcal{F}_{m}^{\prime} \mathcal{C}\right)$ :

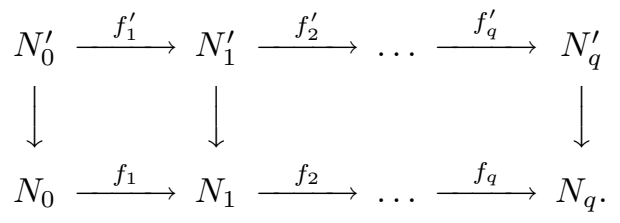

Because the vertical morphisms create no components of augmentation $m$, the decompositions of $N_{q}^{\prime}$ and $N_{q}$ must be compatible under $N_{q}^{\prime} \rightarrow N_{q}$, and so (7.3) must actually be isomorphic to the coproduct of morphisms between chains

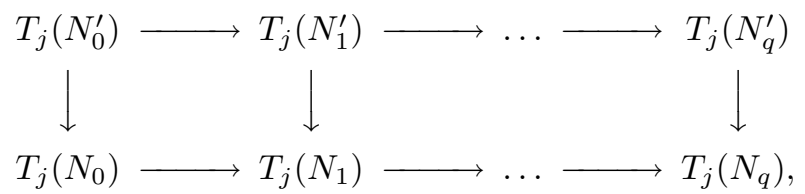

no matter what choices are made at the various stages of the construction. The proposition follows.

The proof of Theorem 7.1 now follows easily.

Proof of Theorem 7.1. We argue by induction on $m$. Clearly $\mathcal{F}_{0} \mathcal{C} \cong \mathcal{C}=\mathcal{K}_{0} \mathcal{C}$. Assume for the inductive hypothesis that there is an augmented weak equivalence $\mathcal{F}_{m-1} \mathcal{C} \simeq \mathcal{K}_{m-1} \mathcal{C}$. It follows from Lemma 7.3, Proposition 7.4, and Proposition 7.8 that there is a chain of monoidal functors inducing a weak equivalence of $\Gamma$-spaces

$$
\left|\mathcal{F}_{m} \mathcal{C}\right| \simeq\left|\operatorname{Bar}\left(\mathcal{F}_{m-1} \mathcal{C}, \operatorname{Free}\left(\mathcal{F}_{m-1} \mathcal{C}_{m}\right), \mathcal{S}_{m}\right)\right|
$$


By Proposition 2.5, it follows that there is a chain of monoidal functors, inducing a equivalence of $\Gamma$-spaces

$$
\left|\mathcal{F}_{m} \mathcal{C}\right| \simeq\left|\mathcal{F}_{m-1} \mathcal{C} \otimes_{\text {Free }\left(\mathcal{F}_{m-1} \mathcal{C}_{m}\right)} \mathcal{S}_{m}\right|
$$

The weak equivalence $\mathcal{F}_{m-1} \mathcal{C} \simeq \mathcal{K}_{m-1} \mathcal{C}$ of the inductive hypothesis restricts to a weak equivalence $\mathcal{F}_{m-1} \mathcal{C}_{m} \simeq \mathcal{K}_{m-1} \mathcal{C}_{m}$. It follows that

$$
\mathcal{F}_{m-1} \mathcal{C} \otimes_{\text {Free }\left(\mathcal{F}_{m-1} \mathcal{C}_{m}\right)} \mathcal{S}_{m} \simeq \mathcal{K}_{m-1} \mathcal{C} \otimes_{\text {Free }\left(\mathcal{K}_{m-1} \mathcal{C}_{m}\right)} \mathcal{S}_{m}=\mathcal{K}_{m} \mathcal{C}
$$

So, we have obtained a chain of weak equivalences, via monoidal functors,

$$
\mathcal{F}_{m} \mathcal{C} \simeq \mathcal{K}_{m} \mathcal{C}
$$

\section{ORBIT CATEGORIES}

In this section we make additional assumptions, over and above those made in Sections 4 and 5, that allow us to relate the subquotients of our filtration to certain classifying spaces of collections of subgroups, a group-theoretic construction. This relates the current work to the program of [11] and [12] and allows us to establish that our construction gives the symmetric powers of spheres when applied to finite pointed sets. We continue the notation of the previous sections: $\mathcal{B}$ is a monogenic category satisfying Axiom 5.1 (Injectivity Axiom), and $\mathcal{C}$ is a category of admissible isomorphisms in $\mathcal{B}$. Recall that $G_{n}$ denotes the group of automorphisms of $g^{n}$ in $\mathcal{C}$.

Definition 8.1. Let $N=n+n_{1}+\cdots+n_{t}$. The identification $g^{N}=g^{n} \oplus g^{n_{1}} \oplus$ $\cdots \oplus g^{n_{t}}$ defines an inclusion of groups

$$
G_{n_{1}} \times \cdots \times G_{n_{t}} \hookrightarrow G_{N}
$$

and we define a standard subgroup of $G_{N}$ to be a subgroup conjugate to the image of such an inclusion. We say that the standard subgroup $G_{n_{1}} \times \cdots \times G_{n_{t}}$ has filtration $m$ if $n_{1}, \ldots, n_{t} \leq m$.

Recall that a "collection" $\mathcal{H}$ of subgroups of a group $G$ is a set of subgroups that is closed under conjugation [5]. The classifying space $B \mathcal{H}$ of the collection is the nerve of the category whose objects are orbits $G / H$ for $H \in \mathcal{H}$ and whose maps are $G$-equivariant maps. For example, for a fixed $n$, the set of standard subgroups of $G_{n}$ of filtration $m$ is a collection, which we denote $\mathcal{H}_{m}\left(G_{n}\right)$, and we denote its classifying space $B \mathcal{H}_{m}\left(G_{n}\right)$.

The additional assumptions for this section are detailed below as Axioms 8.5 and 8.7, and the goal of the section is the following theorem and its corollaries, which relate Construction 3.8 to group-theoretic constructions. The main technical tool in the proofs is Proposition 8.9.

Theorem 8.2. Let $\mathcal{C}$ be a monogenic category satisfying Axioms 5.1, 8.5, and 8.7. Then $B \mathcal{K}_{m} \mathcal{C}$ is monoidally equivalent to $\coprod_{n} B \mathcal{H}_{m}\left(G_{n}\right)$.

Corollary 8.3. Given a category $\mathcal{C}$ as in Theorem 8.2, let $\mathcal{R}_{m}$ be the collection $\mathcal{H}_{m-1}\left(G_{m}\right)$, and let $\epsilon: \Sigma^{\infty}\left(B \mathcal{R}_{m}\right)_{+} \rightarrow \mathbf{S}$ be induced by the map $B \mathcal{R}_{m} \rightarrow *$. Then

$$
A_{m} \simeq \operatorname{hocolim}\left(\begin{array}{c}
\Sigma^{\infty}\left(B \mathcal{R}_{m}\right)_{+} \longrightarrow A_{m-1} \\
\epsilon \downarrow \\
\mathbf{S}
\end{array}\right) .
$$


Corollary 8.4. Let $A_{0} \rightarrow A_{1} \rightarrow \ldots$ be the sequence of spectra resulting from the application of Construction 3.8 to the category of finite sets and isomorphisms. Then $A_{m} \simeq \operatorname{Sp}^{m}(\mathbf{S})$.

To prove these results, we need to identify the set of morphisms

$$
\left[n ; n_{1}, \ldots, n_{t}\right] \rightarrow\left[r ; r_{1}, \ldots, r_{u}\right]
$$

in $\mathcal{F}_{\infty} \mathcal{C}$ as a certain set of equivariant maps in an orbit category of the groups $G_{N}$, where $N=n+n_{1}+\cdots+n_{t}$. The first additional assumption that we need on the underlying category $\mathcal{B}$ has to do with the morphism sets.

Axiom 8.5 (Fixed Point Axiom). The pointed category $\mathcal{B}$ satisfies the property that given two objects $U, V \in \mathcal{B}$, the only element of $\operatorname{Morph}_{\mathcal{B}}(U, V)$ that is fixed under the action of $\operatorname{Aut}_{\mathcal{C}}(V)$ is the null map, $U \rightarrow 0 \rightarrow V$.

The axiom clearly holds for the category of finite-dimensional vector spaces over the real or complex numbers and isometric maps. It does not quite hold for the category of finite pointed sets, because if $V$ is the set with one non-basepoint element, then $\operatorname{Aut}_{\mathcal{C}}(V)$ is the trivial group, so any morphism $U \rightarrow V$ is invariant under the automorphisms of $V$. However, the axiom does hold if $V$ is a set with more than one element, and the main conclusions that we wish to draw do hold for the category of finite sets. (See the proof of Corollary 8.4.) Similarly, for a general ring $R$, the category of free $R$-modules may not satisfy Axiom 8.5 if, for example, $\mathrm{GL}_{1}(R)$ is the trivial group. However, the axiom is satisfied if $R$ is an integral domain in which $2 \neq 0$.

We require one last property of the categories $\mathcal{B}$ and $\mathcal{C}$, which we assume for the remainder of this section, along with Axioms 5.1 and 8.5. In order to set it out, we need the following definitions.

Definition 8.6. Let $i: U \hookrightarrow V \oplus V^{\prime}$ be a representative cofibration for a subobject $U$ of $V \oplus V^{\prime}$ in $\mathcal{B}$.

(1) We say that the subobject $U$ is invariant under $\operatorname{Aut}_{\mathcal{C}}(V)$ if for all $\sigma \in$ $\operatorname{Aut}_{\mathcal{C}}(V)$, there exists $\tau \in \operatorname{Aut}_{\mathcal{C}}(U)$ such that $i \circ \tau=\left(\sigma \oplus \operatorname{id}_{V^{\prime}}\right) \circ i$.

(2) We say that $U$ contains $V$ if there exists an object $V^{\prime \prime}$, a $\mathcal{C}$-admissible isomorphism $U \rightarrow V \oplus V^{\prime \prime}$, and a cofibration $V^{\prime \prime} \hookrightarrow V^{\prime}$ such that $i$ factors as a composite $U \cong V \oplus V^{\prime \prime} \hookrightarrow V \oplus V^{\prime}$.

(3) We say that $U$ is contained in $V^{\prime}$ if $i$ factors as a composite of a cofibration $U \hookrightarrow V^{\prime}$ with the standard inclusion $V^{\prime} \hookrightarrow V \oplus V^{\prime}$.

Axiom 8.7 (Invariance Axiom). The category $\mathcal{B}$ satisfies the property that if $i: U \hookrightarrow V \oplus V^{\prime}$ is a subobject that is invariant under $\operatorname{Aut}_{\mathcal{C}}(V)$, then either $U$ contains $V$ or $U$ is contained in $V^{\prime}$.

The categories of finite pointed sets and finite-dimensional real and complex vector spaces satisfy the Invariance Axiom. The case of free $R$-modules deserves its own lemma. (Our thanks to Bill Dwyer for a helpful conversation on this subject.)

Lemma 8.8. If $R$ is an integral domain with $2 \neq 0$, then the category of finitelygenerated free modules over $R$ satisfies Axiom 8.7.

Proof. Let $U$ be a subobject of $V \oplus V^{\prime}$ that is invariant under the action of GL $(V)$. There is an isomorphism $i: U \oplus U^{\prime} \rightarrow V \oplus V^{\prime}$, where $U, U^{\prime}, V, V^{\prime}$ are free $R$-modules. 
We assume that $i(U)$ is invariant under $\mathrm{GL}(V)$ and is not contained in $V^{\prime}$, and we prove that $i(U)$ contains $V$.

We first assert that if $r \in R$ is nonzero and $(r v, 0) \in i(U)$, then $(v, 0) \in i(U)$. For suppose that $(r v, 0)=i(u, 0)$, and suppose that $(v, 0)$ has preimage $(a, b)$ under the isomorphism $i$. Since $i(r a, r b)=(r v, 0)=i(u, 0)$, we have $(r a, r b)=(u, 0)$ and thus $r b=0$. Since $r \neq 0$ and $V$ is a free module over the domain $R$, this tells us that $b=0$, and it follows that $(v, 0)=i(a, 0) \in i(U)$.

Our next step is to prove that $i(U)$ contains an element of the form $(v, 0)$ with $v \neq 0$. Since $i(U)$ is not contained in $V^{\prime}$, it contains an element of the form $\left(v, v^{\prime}\right)$ with $v \neq 0$. By invariance under $\operatorname{GL}(V)$, we know $i(U)$ contains $\left(-v, v^{\prime}\right)$, and therefore also $(2 v, 0)$. Then $(v, 0) \in V \cap i(U)$ by the previous argument, since 2 is nonzero in $R$.

Since $V$ is a finitely-generated free $R$-module, we can choose a basis for $V$ and write $v=\left(r_{1}, \ldots, r_{n}\right)$ where $r_{1}, \ldots, r_{n} \in R$. Since $v \neq 0$, suppose that $r_{1} \neq 0$. By invariance of $i(U)$ under $\mathrm{GL}(V)$, we also know $\left(r_{1}, r_{2}+r_{1}, \ldots, r_{n}\right) \in V \cap i(U)$, and so $\left(0, r_{1}, 0, \ldots, 0\right) \in V \cap i(U)$. But $r_{1} \neq 0$, so we find that $(0,1,0 \ldots, 0) \in V \cap i(U)$. Since all permutations of the standard basis elements of $V$ are in $\operatorname{GL}(V)$, we find that all of the standard basis elements of $V$ are in $i(U)$, and we conclude that $i(U)$ contains all of $V$, as required.

This completes the new assumptions that we need to make on the categories $\mathcal{B}$ and $\mathcal{C}$. We need more notation in order to describe how the category $\mathcal{F}_{\infty} \mathcal{C}$ is supposed to correspond to an orbit category. Given an object $\left[n ; n_{1}, \ldots, n_{t}\right]$ of $\mathcal{F}_{\infty} \mathcal{C}$, let $N=n+n_{1}+\cdots+n_{t}$, let $G_{n_{\bullet}}=G_{n_{1}} \times \cdots \times G_{n_{t}}$, and let $\mathcal{O}_{\infty} \mathcal{C}$ be the category whose objects are orbit spaces $G / G_{n}$. for some $\left[n ; n_{1}, \ldots, n_{t}\right]$, with morphisms being $G_{N}$-equivariant maps. That is, if $r, r_{1}, \ldots, r_{u}$ are integers with $n+n_{1}+\cdots+n_{t}=r+r_{1}+\cdots+r_{u}$, and $G=G_{n+n_{1}+\cdots+n_{t}}=G_{r+r_{1}+\cdots+r_{u}}$, then $G / G_{n}$ and $G / G_{r_{\bullet}}$ are two objects of $\mathcal{O}_{\infty} \mathcal{C}$ and

$$
\operatorname{Morph}_{\mathcal{O}_{\infty} \mathcal{C}}\left(G / G_{n_{\bullet}}, G / G_{r_{\bullet}}\right)=\operatorname{map}_{G}\left(G / G_{n_{\bullet}}, G / G_{r_{\bullet}}\right) \text {. }
$$

Similarly, let $\mathcal{O}_{m} \mathcal{C}$ be the full subcategory of $\mathcal{O}_{\infty} \mathcal{C}$ whose objects are orbit spaces $G / G_{n}$. where $n_{i} \leq m$ for all $1 \leq i \leq t$.

Recall from Remark 4.11 that

$$
\operatorname{Morph}_{\mathcal{F}_{m} \mathcal{C}}\left(\left[n ; n_{1}, \ldots, n_{t}\right],\left[r ; r_{1}, \ldots, r_{u}\right]\right) \subset \operatorname{map}_{G}\left(G / G_{n_{\bullet}}, G / G_{r_{\bullet}}\right) .
$$

It follows that for every $m$, including $m=\infty$, there is a natural functor $\mathcal{F}_{m} \mathcal{C} \rightarrow$ $\mathcal{O}_{m} \mathcal{C}$ sending $\left[n ; n_{1}, \ldots, n_{t}\right]$ to $G / G_{n}$. and inducing the above inclusion on morphisms. Further, $\mathcal{O}_{\infty} \mathcal{C}$ is a symmetric monoidal category, with product given by inducing up the product of two orbits, as in [11], and the functors $\mathcal{F}_{m} \mathcal{C} \rightarrow \mathcal{O}_{m} \mathcal{C}$ are monoidal.

Proposition 8.9. Let $\mathcal{B}$ be a monogenic category that satisfies Axioms 5.1, 8.5 and 8.7, and let $\mathcal{C}$ be a subcategory of admissible isomorphisms. The functor $\mathcal{F}_{\infty} \mathcal{C} \rightarrow$ $\mathcal{O}_{\infty} \mathcal{C}$ sending $\left[n ; n_{1}, \ldots, n_{t}\right]$ to $G_{N} / G_{n}$. induces a bijection on morphism sets.

Proof. We only need to show that the map on morphism sets is surjective. Suppose we have objects $\left[n ; n_{1}, \ldots, n_{t}\right]$ and $\left[r ; r_{1}, \ldots, r_{u}\right]$ with $n+n_{1}+\cdots+n_{t}=r+r_{1}+\cdots+$ $r_{u}$, and let $G=G_{n+n_{1}+\cdots+n_{t}}$. We show that an element of $\operatorname{map}_{G}\left(G / G_{n_{\bullet}}, G / G_{r_{\bullet}}\right)$ comes from a morphism

$$
\left[n ; n_{1}, \ldots, n_{t}\right] \rightarrow\left[r ; r_{1}, \ldots, r_{u}\right]
$$


in $\mathcal{F}_{\infty} \mathcal{C}$.

Choose an element in $\operatorname{map}_{G}\left(G / G_{n_{\bullet}}, G / G_{r_{\bullet}}\right) \cong\left(G / G_{r_{\bullet}}\right)^{G_{n}}$; suppose it is represented by an isomorphism in $G$, say

$$
f: g^{n} \oplus g^{n_{1}} \oplus \cdots \oplus g^{n_{t}} \rightarrow g^{r} \oplus g^{r_{1}} \oplus \cdots \oplus g^{r_{u}} .
$$

(Throughout this proof "isomorphism" means "C-admissible isomorphism.") The element $f \in G$ conjugates $G_{n_{\bullet}}$ into $G_{r_{\bullet}}$, and so has the property that if $\psi \in G_{n_{\bullet}}$, then there exists $\phi \in G_{r_{\bullet}}$ such that the following diagram commutes:

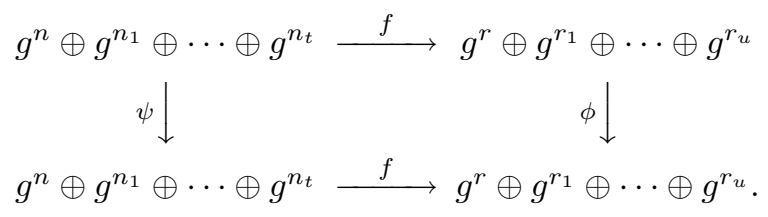

On the other hand, a morphism $\left[n ; n_{1}, \ldots, n_{t}\right] \rightarrow\left[r ; r_{1}, \ldots, r_{u}\right]$ is an equivalence class of isomorphisms

$$
g^{n} \oplus g^{n_{1}} \oplus \cdots \oplus g^{n_{t}} \rightarrow g^{r} \oplus g^{r_{1}} \oplus \cdots \oplus g^{r_{u}}
$$

under the action of $\prod \operatorname{Aut}\left(g^{r_{i}}\right)$. We need to do three things:

(1) find an appropriate function $\alpha: \underline{t} \rightarrow \underline{u}$;

(2) establish that $f$ is compatible with $\alpha$, i.e., for each $j \in \underline{u}$, the restriction $\left.f\right|_{\oplus_{i \in \alpha^{-1}(j)}} g^{n_{i}}$ factors through the inclusion $g^{r_{j}} \hookrightarrow g^{r} \oplus g^{r_{1}} \oplus \cdots \oplus g^{r_{u}}$;

(3) establish that $\left.f\right|_{g^{n}}$ factors as $g^{n} \cong g^{r} \oplus g^{e_{1}} \oplus \cdots \oplus g^{e_{u}} \hookrightarrow g^{r} \oplus g^{r_{1}} \oplus \cdots \oplus g^{r_{u}}$.

We begin by constructing an appropriate $\alpha$. Consider $f^{-1}: g^{r} \oplus g^{r_{1}} \oplus \cdots \oplus g^{r_{u}} \rightarrow$ $g^{n} \oplus g^{n_{1}} \oplus \cdots \oplus g^{n_{t}}$, and fix $j \in \underline{u}$. The restriction of $f^{-1}$ to $g^{r_{j}} \rightarrow g^{n} \oplus g^{n_{1}} \oplus \cdots \oplus g^{n_{t}}$ is a cofibration, and we define $f^{-1}\left(g^{r_{j}}\right)$ to be the corresponding subobject of $g^{n} \oplus$ $g^{n_{1}} \oplus \cdots \oplus g^{n_{t}}$. Note that the subobject $f^{-1}\left(g^{r_{j}}\right)$ is not dependent on the specific representative $f$, since any other representative is of the form $\phi \circ f$ for $\phi \in G_{r_{\bullet}}$, and the indeterminacy is taken care of in the equivalence relation of cofibrations that defines a subobject. Furthermore, because $f$ conjugates $G_{n}$, into $G_{r_{\bullet}}$, we see that $f^{-1}\left(g^{r_{j}}\right)$ is invariant under the action of $\prod \operatorname{Aut}\left(g^{n_{i}}\right)$, as expressed by (8.1).

We now consider $i=1, \ldots, t$ one element at a time, and decide whether or not each one will be in $\alpha^{-1}(j)$. Beginning with $i=1$, we know that $f^{-1}\left(g^{r_{j}}\right)$ is invariant under $\operatorname{Aut}\left(g^{n_{1}}\right)$. We apply Axiom 8.7, and one of two things can happen.

- The first possibility is that $f^{-1}\left(g^{r_{j}}\right)$ contains $g^{n_{1}}$. In this case, there exists an isomorphism $h: g^{r_{j}} \cong g^{n_{1}} \oplus g^{n_{1}^{\prime}}$ and a direct sum of of the identity map on $g^{n_{1}}$ with another cofibration

$$
g^{n_{1}} \oplus g^{n_{1}^{\prime}} \hookrightarrow g^{n_{1}} \oplus\left(g^{n} \oplus g^{n_{2}} \oplus \cdots \oplus g^{n_{t}}\right)
$$

such that $\left.f^{-1}\right|_{g^{r_{j}}}$ factors through the sum. We define $\alpha(1)=j$, accepting 1 as an element of $\alpha^{-1}(j)$.

To iterate, we need to show that the subobject defined by $g^{n_{1}^{\prime}} \hookrightarrow g^{n} \oplus$ $g^{n_{2}} \oplus \cdots \oplus g^{n_{t}}$ is still invariant under the action of any element $\psi \in$ $\prod_{i=2}^{t} \operatorname{Aut}\left(g^{n_{i}}\right)$. Let $\phi$ be the element corresponding to $\psi$ that is given 
by (8.1), say with component $\phi_{j}: g^{r_{j}} \rightarrow g^{r_{j}}$. We have a commuting diagram

$$
\begin{aligned}
& g^{r_{j}} \stackrel{h}{\cong} g^{n_{1}} \oplus g^{n_{1}^{\prime}} \longrightarrow g^{n_{1}} \oplus\left(g^{n} \oplus g^{n_{2}} \oplus \cdots \oplus g^{n_{t}}\right) \\
& \phi_{j} \downarrow \cong \quad \psi \downarrow \cong \\
& g^{r_{j}} \stackrel{h}{\cong} g^{n_{1}} \oplus g^{n_{1}^{\prime}} \longrightarrow g^{n_{1}} \oplus\left(g^{n} \oplus g^{n_{2}} \oplus \cdots \oplus g^{n_{t}}\right) .
\end{aligned}
$$

Invariance will now follow once we show that $h \phi_{j} h^{-1}$ is the coproduct of the identity map on $g^{n_{1}}$ with an isomorphism on $g^{n_{1}^{\prime}}$. This last follows from a calculation using Lemma 5.2; the input for the lemma is provided by going clockwise around the diagram instead and using the fact that if the composite of a map with a cofibration is null, then the original map is null as well.

- The second possibility is that $f^{-1}\left(g^{r_{j}}\right)$ is contained in $g^{n} \oplus g^{n_{2}} \oplus \cdots \oplus g^{n_{t}}$. This gives us a cofibration $g^{r_{j}} \hookrightarrow g^{n} \oplus g^{n_{2}} \oplus \cdots \oplus g^{n_{t}}$ through which $f^{-1} \mid g^{r_{j}}$ factors, and again, this is invariant under $\prod_{i=2}^{t} \operatorname{Aut}\left(g^{n_{i}}\right)$. Observe that this implies that the composite

$$
g^{r_{j}} \hookrightarrow g^{r} \oplus g^{r_{1}} \oplus \cdots \oplus g^{r_{u}} \rightarrow g^{n} \oplus g^{n_{1}} \oplus \cdots \oplus g^{n_{t}} \rightarrow g^{n_{1}}
$$

is null. In this case, we reject 1 as an element of $\alpha^{-1}(j)$.

We iterate this procedure $t$ times, each time accepting or discarding $i$ as an element of $\alpha^{-1}(j)$. After we have completed the $t$ iterations, we define $e_{j}=r_{j}-$ $\sum_{i \in \alpha^{-1}(j)} n_{i}$, and we have an isomorphism $\zeta_{j}: g^{r_{j}} \cong g^{n_{i_{1}}} \oplus \cdots \oplus g^{n_{i_{k}}} \oplus g^{e_{j}}$ and a cofibration $g^{e_{j}} \hookrightarrow g^{n}$ such that $\left.f^{-1}\right|_{g^{r_{j}}}$ is the composition of $\zeta_{j}$ with the direct sum of the natural inclusions on $g^{n_{i_{1}}} \oplus \cdots \oplus g^{n_{i_{k}}}$ and a cofibration $g^{e_{j}} \hookrightarrow g^{n}$. Note that we do not yet know that $\alpha$ is a well-defined function, since it is not obvious that the sets $\alpha^{-1}(j)$ are disjoint for different $j$, nor that each $i \in \underline{t}$ is in one of the sets $\alpha^{-1}(j)$. We will check these below.

Our next step is to prove that $\left.f^{-1}\right|_{g^{r}}$ factors through $g^{n}$, for which we need Axiom 8.5. We assert that for each $i$, the composite morphism $g^{r} \rightarrow g^{n} \oplus g^{n_{1}} \oplus$ $\cdots \oplus g^{n_{t}} \rightarrow g^{n_{i}}$ is invariant under $\operatorname{Aut}\left(g^{n_{i}}\right)$, and therefore is null by Axiom 8.5. This follows from diagram (8.1), since no $\phi \in G_{r_{\text {}}}$. has any effect on $g^{r}$, and thus any element $\psi \in G_{n_{\bullet}}$ must fix the actual morphism $g^{r} \rightarrow g^{n} \oplus g^{n_{1}} \oplus \cdots \oplus g^{n_{t}}$, and not just the subobject it defines. Therefore, by applying Corollary 5.3 we find that $\left.f^{-1}\right|_{g^{r}}$ factors through $g^{n} \hookrightarrow g^{n} \oplus g^{n_{1}} \oplus \cdots \oplus g^{n_{t}}$.

The result of the preceding constructions is that we have a commuting diagram

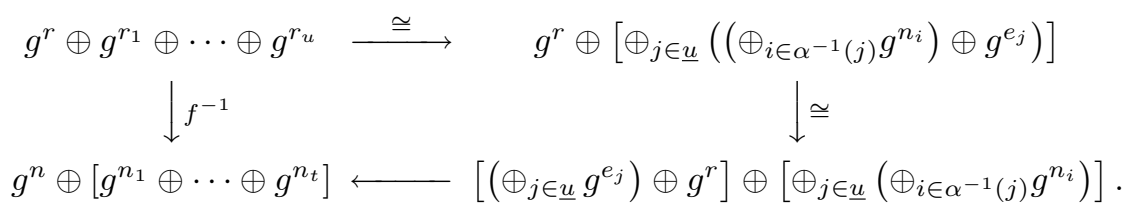

The top row is an isomorphism, since for each $j$, the map $g^{r_{j}} \rightarrow\left(\oplus_{i \in \alpha^{-1}(j)} g^{n_{i}}\right) \oplus g^{e_{j}}$ is an isomorphism by construction. The right vertical map is an isomorphism because it simply reshuffles and reassociates the terms. Thus the bottom row is also an isomorphism, and, by construction, it is the coproduct of two maps (with the association shown), each of which is therefore an isomorphism by Condition (4) in Definition 4.2. We are immediately entitled to conclude that $\left.f\right|_{g^{n}}$ factors as 
$g^{n} \cong g^{r} \oplus g^{e_{1}} \oplus \cdots \oplus g^{e_{u}} \hookrightarrow g^{r} \oplus g^{r_{1}} \oplus \cdots \oplus g^{r_{u}}$, the third condition we needed in order for $f$ to represent a morphism in $\mathcal{F}_{\infty} \mathcal{C}$.

Finally, to check that $\alpha$ is indeed a well-defined function and that $f$ is compatible with $\alpha$, we consider the other summand of the bottom row of the diagram,

$$
\left(\oplus_{i \in \alpha^{-1}(1)} g^{n_{i}}\right) \oplus \cdots \oplus\left(\oplus_{i \in \alpha^{-1}(u)} g^{n_{i}}\right) \rightarrow g^{n_{1}} \oplus \cdots \oplus g^{n_{t}}
$$

which is also an isomorphism by Condition (4) in Definition 4.2. Notice that this map is a coproduct of natural inclusions of summands, though it is in principle possible for some of the summands to appear on the left more than once, while others might not appear at all. In fact, in order to know that $\alpha$ is a well-defined function $\underline{t} \rightarrow \underline{u}$, we need to prove precisely this does not happen, that is, we need to know that $\alpha^{-1}(1), \ldots, \alpha^{-1}(u)$ is actually a partition of $\underline{t}$. We assert that the union of $\alpha^{-1}(1), \ldots, \alpha^{-1}(u)$ is $\underline{t}$, which is to say that every $n_{i}$ appears on the left of (8.2), because otherwise the isomorphism (8.2) would factor through a proper subobject of $g^{n_{1}} \oplus \cdots \oplus g^{n_{t}}$, which would have lower dimension, violating the assumption of invariance of dimension in $\mathcal{B}$. The proof is concluded by noting that the total dimension on each side of the isomorphism must be the same, and so each $n_{i}$ can only appear once on the left side, establishing that the sets $\alpha^{-1}(j)$ are disjoint for different values of $j$.

The proofs of Theorem 8.2 and Corollaries 8.3 and 8.4 follow easily.

Proof of Theorem 8.2. The functor $\mathcal{F}_{\infty} \mathcal{C} \rightarrow \mathcal{O}_{\infty} \mathcal{C}$ is a monomorphism on objects, and every object of $\mathcal{O}_{\infty} \mathcal{C}$ is isomorphic to one of the form $G / G_{n_{\bullet}}$. By Proposition 8.9 , the image of $\mathcal{F}_{\infty} \mathcal{C} \rightarrow \mathcal{O}_{\infty} \mathcal{C}$ is a full subcategory of $\mathcal{O}_{\infty} \mathcal{C}$.

Proof of Corollary 8.3. By Theorem 3.9, we have a diagram

$$
A_{m} \simeq \operatorname{hocolim}\left(\begin{array}{c}
\sum^{\infty}\left(B \mathcal{K}_{m-1} \mathcal{C}_{m}\right)_{+} \longrightarrow A_{m-1} \\
\epsilon \downarrow \\
\mathbf{S}
\end{array}\right)
$$

so we must show that $B \mathcal{K}_{m-1} \mathcal{C}_{m} \simeq B \mathcal{R}_{m}$. However, $B \mathcal{R}_{m}=B \mathcal{H}_{m-1}\left(G_{m}\right)$, and by Proposition 8.9, $B \mathcal{H}_{m-1}\left(G_{m}\right) \simeq B \mathcal{F}_{m-1} C_{m}$. Finally, Theorem 7.1 says that $B \mathcal{F}_{m-1} C_{m} \simeq B \mathcal{K}_{m-1} \mathcal{C}_{m}$.

Proof of Corollary 8.4. Strictly speaking, Axiom 8.5 does not hold in the case that $\mathcal{B}$ is finite pointed sets; however, it still is true that $\mathcal{F}_{m} \mathcal{C}$ and $\mathcal{O}_{m} \mathcal{C}$ are connected by a chain of monoidal functors, each inducing an equivalence of classifying spaces. One way to see this is to recall that $\mathcal{F}_{m} \mathcal{C}$ is connected in this way to the category of "finite sets with multiplicity at most $m$ " (see Example 4.10), and it is easy to see directly that the latter category is connected in the appropriate way to $\mathcal{O}_{m} \mathcal{C}$.

Let $F^{\prime}\left(\Sigma_{m}\right)$ denote the collection of all nontransitive subgroups of $\Sigma_{m}$. (The collection $F^{\prime}\left(\Sigma_{m}\right)$ is actually a "family" of subgroups, meaning that it is closed under taking subgroups as well as under conjugation, but that does not concern us here.) The corollary follows from [12] once we know that $B F^{\prime}\left(\Sigma_{m}\right) \simeq B \mathcal{H}_{m-1}\left(\Sigma_{m}\right)$, which is true because to every nontransitive subgroup of $\Sigma_{m}$, we can associate the unique minimal standard subgroup of filtration $m-1$ that contains it, and the association is compatible with conjugation. 


\section{Relationship With The POSET OF DIRECT-SUM DECOMPOSITIONS}

For the remainder of the paper, we focus our attention on the example of finitedimensional unitary vector spaces, and its relationship with the example of finite sets, and we use $A_{m}$ specifically to refer to the result of applying our construction to the former. Our goal in this section is to provide another description of the filtration subquotients $A_{m} / A_{m-1}$ in the unitary case, in preparation for relating our filtration to the calculus of functors. In particular, we prove Theorem 9.5, which expresses the subquotients of our filtration of $b u$ in terms of certain spaces of direct-sum decompositions, and Theorem 9.7, which codifies their behavior at a prime $p$.

We need some definitions. Recall that by a subgroup of a Lie group we always mean a closed subgroup, and that the standard subgroups of $U(m)$ are those conjugate to $U\left(m_{1}\right) \times \cdots \times U\left(m_{s}\right)$. (See Definition 8.1.)

Definition 9.1. Let $H$ be a subgroup of $U(m)$ for $m \geq 1$.

(1) We say $H$ is bad if the action of $H$ on $\mathbb{C}^{m}$ breaks $\mathbb{C}^{m}$ into a sum $\mathbb{C}^{m} \cong \gamma^{\oplus k}$ where $\gamma$ is irreducible and nontrivial. That is, $H$ is bad if it acts isotypically with nontrivial irreducibles.

(2) If $H$ is not bad, then we call $H$ good.

(3) If $H$ is a standard subgroup conjugate to one of the form $\prod_{i=1}^{s} U\left(m_{i}\right)$ where $\sum m_{i}=m$ and $s>1$, then we call $H$ complete.

Example 9.2. The trivial subgroup $\{e\} \subseteq U(m)$ is good. The center $S^{1}=$ $Z(U(m))$ is bad. The whole group $U(m)$ is bad.

We will work with the following collections of subgroups of $U(m)$ :

$$
\begin{aligned}
\mathcal{G}_{m} & =\{\text { all good subgroups of } U(m)\} \\
\mathcal{R}_{m} & =\{\text { proper standard subgroups of } U(m)\} \\
\mathcal{L}_{m} & =\{\text { complete subgroups of } U(m)\} .
\end{aligned}
$$

We note that $\mathcal{L}_{m}$, regarded as a poset under inclusion, can be identified with the poset of proper direct-sum decompositions of $\mathbb{C}^{m}$ by taking a subgroup to the directsum decomposition given by its irreducible subspaces. Let $\mathcal{G}_{m, \text { ntrv }}$ and $\mathcal{R}_{m, \text { ntrv }}$ be the collections consisting of nontrivial elements of $\mathcal{G}_{m}$ and $\mathcal{R}_{m}$, respectively. Note that there are inclusions $\mathcal{L}_{m} \hookrightarrow \mathcal{R}_{m} \hookrightarrow \mathcal{G}_{m}$. There are also maps back, $\mathcal{G}_{m, \text { ntrv }} \rightarrow \mathcal{R}_{m, \text { ntrv }} \rightarrow \mathcal{L}_{m}$ which, although not maps of posets, turn out to be useful in the proofs of Propositions 9.8 and 9.9, where they are described in more detail.

Remark 9.3. The poset of proper direct-sum decompositions of $\mathbb{C}^{m}$, was studied in [1]. The space denoted $\left|\mathcal{L}_{m}\right|$ in this paper is homeomorphic to the space denoted $\tilde{L}_{m}$, in [1], page 458 , while the space $\left|\mathcal{L}_{m}\right|^{\diamond}$ introduced below is homeomorphic to the space denoted $L_{m}\left(\right.$ or $\left.L_{m}^{\mathbb{C}}\right)$ in [1].

Given a collection $\mathcal{H}$ of subgroups of a group $G$, let $E \mathcal{H}$ denote the universal space of the collection. The space $E \mathcal{H}$ can be constructed as the nerve of the category whose objects are pairs $(G / H, x)$ with $H \in \mathcal{H}$ and $x \in G / H$, and whose morphisms $(G / H, x) \rightarrow\left(G / H^{\prime}, x^{\prime}\right)$ are $G$-equivariant maps $G / H \rightarrow G / H^{\prime}$ taking $x$ to $x^{\prime}$. The space $E \mathcal{H}$ is characterized by the following two properties: (1) $E \mathcal{H}^{K}$ is contractible for all $K \in \mathcal{H}$, and (2) all the isotropy groups of $E \mathcal{H}$ are elements of $\mathcal{H}$. (See [2], Lemma 2.3.) The classifying space $B \mathcal{H}$ of the collection $\mathcal{H}$ is the 
orbit space of $E \mathcal{H}$ under the action of $G$. Recall from [5], 2.12 that there is an equivariant map $E \mathcal{H} \rightarrow|\mathcal{H}|$ that is a non-equivariant homotopy equivalence. ${ }^{2}$ (We will call such a map an equivariant weak equivalence for short.) In particular, there is an equivariant weak equivalence $E \mathcal{L}_{m} \simeq\left|\mathcal{L}_{m}\right|$.

Given a space $X$, let $X^{\diamond}$ denote its unreduced suspension. If $X$ is a pointed $G$-space, let $X_{\tilde{h} G}$ denote the based homotopy orbits of the action of $G$ on $X$ : namely $X_{\tilde{h} G}=\left(E G \times{ }_{G} X\right) /(B G \times *)$. We let $U(m)$ act on $S^{2 m}$ by the one-point compactification of of its action on $\mathbb{C}^{m}$. Thus the action of $U(m)$ fixes the basepoint of $S^{2 m}$, which is the point at infinity, as well as fixing the origin. Our main technical goal in this section is to prove the following theorem.

\section{Theorem 9.4.}

$$
\Sigma B \mathcal{R}_{m} \simeq \Sigma\left(E \mathcal{L}_{m}^{\diamond} \wedge S^{2 m}\right)_{\tilde{h} U(m)} \simeq \Sigma\left(\left|\mathcal{L}_{m}\right|^{\diamond} \wedge S^{2 m}\right)_{\tilde{h} U(m)}
$$

The proof of the theorem is deferred to later in the section, following the proof of Corollary 9.14. Let us first note some consequences of the theorem for our filtration of $b u$.

Theorem 9.5. There is an equivalence of spectra

$$
A_{m} / A_{m-1} \simeq \Sigma^{\infty} \Sigma\left(\left|\mathcal{L}_{m}\right|^{\diamond} \wedge S^{2 m}\right)_{\tilde{h} U(m)}
$$

where $\mathcal{L}_{m}$ is the category of proper direct-sum decompositions of $\mathbb{C}^{m}$.

Proof. Follows immediately from Corollary 8.3 and Theorem 9.4.

The complexes $\left|\mathcal{L}_{m}\right|^{\diamond}$ were first introduced in [1] because they play an important role in Weiss's orthogonal calculus. They have many interesting properties, some of which we will discuss in the next section, where we pursue further the relationship of our present work with the calculus of functors. For starters, we have the following proposition.

Proposition 9.6. The complex $\left|\mathcal{L}_{m}\right|^{\diamond}$ is rationally trivial for $m>1$. For a prime $p$, the complex $\left|\mathcal{L}_{m}\right|^{\diamond}$ is trivial at $p$ unless $m$ is a power of $p$. In particular, $\left|\mathcal{L}_{m}\right|^{\diamond}$ is contractible unless $m$ is a power of a prime.

Proof. This is implicit in [1]. In fact, two independent proofs of this result are given there, but the statement is never made explicit. Let $S^{\operatorname{ad}_{m}}$ be the one-point compactification of the adjoint representation of $U(m)$. Perhaps the quickest way to get the results of the proposition is to start with the first sentence of [1], Theorem 4(a) and the first two sentences of [1], Theorem 4(b). When unraveled, these say that

$$
\operatorname{map}\left(\left|\mathcal{L}_{m}\right|^{\diamond}, \Sigma^{\infty} S^{\operatorname{ad}_{m}}\right)
$$

is contractible rationally if $m \geq 2$, is contractible unless $m$ is a power of a prime, and is all $p$-torsion when $m$ is a positive power of $p$.

Since $\left|\mathcal{L}_{m}\right|^{\diamond}$ is a finite complex, it follows by Spanier-Whitehead duality that $\Sigma^{\infty}\left|\mathcal{L}_{m}\right|^{\diamond}$ is rationally acyclic for $m \geq 2$, and is acyclic at $p$ unless $m$ is a power of $p$. Therefore $\left|\mathcal{L}_{m}\right|^{\diamond}$ has the same properties. Since $\left|\mathcal{L}_{m}\right|$ is connected, $\left|\mathcal{L}_{m}\right|^{\diamond}$ is simply connected, and acyclicity implies contractibility.

Another way to prove the result is to use [1], Theorem 11, and then apply the theory of homology approximations.

\footnotetext{
${ }^{2}$ The result is only stated for finite groups, but the same argument works for compact Lie groups.
} 
As a consequence, we have the following theorem.

Theorem 9.7. Let $p$ be a prime. The successive quotients $A_{m} / A_{m-1}$ are trivial at $p$ except when $m=p^{k}$ for some $k \geq 1$, and are rationally trivial for $m \geq 2$.

We need a series of results to prepare the ground for proving Theorem 9.4. The collections $\mathcal{G}_{m}, \mathcal{R}_{m}$, and $\mathcal{L}_{m}$ have actions of $U(m)$ by conjugation, and the inclusions

$$
\mathcal{L}_{m} \hookrightarrow \mathcal{R}_{m} \hookrightarrow \mathcal{G}_{m}
$$

are $U(m)$-equivariant. The following two propositions give the foundation for the relationship between $\mathcal{G}_{m}, \mathcal{R}_{m}$ and $\mathcal{L}_{m}$ that we need. Their proofs are very similar but not particularly enlightening, and are given at the end of the section.

Proposition 9.8. The map

$$
E \mathcal{L}_{m} \hookrightarrow E \mathcal{R}_{m, \mathrm{ntrv}}
$$

is a $U(m)$-equivalence.

Proposition 9.9. The map

$$
E \mathcal{R}_{m} \hookrightarrow E \mathcal{G}_{m}
$$

is a $U(m)$-equivalence.

Corollary 9.10. The map

$$
E \mathcal{R}_{m, \mathrm{ntrv}} \rightarrow E \mathcal{G}_{m, \mathrm{ntrv}}
$$

is a $U(m)$-equivalence.

Proof. We assert that the singular set of $E \mathcal{R}_{m}$ is $E \mathcal{R}_{m, n t r v}$, which is easily verified by checking the isotropy groups of the chains that form the simplices of $E \mathcal{R}_{m}$. Likewise, the singular set of $E \mathcal{G}_{m}$ is $E \mathcal{G}_{m, n t r v}$. The corollary now follows from Proposition 9.9 and [2], Lemma 2.4.

In contemplating the desired equivalence of Theorem 9.4, we see that Proposition 9.8 gives us an equivalence

$$
\Sigma\left(E \mathcal{L}_{m}^{\diamond} \wedge S^{2 m}\right)_{\tilde{h} U(m)} \simeq \Sigma\left(E \mathcal{R}_{m, \mathrm{ntrv}} \diamond \wedge S^{2 m}\right)_{\tilde{h} U(m)} .
$$

Thus we tackle the right-hand side of this equivalence and seek to relate it to $\Sigma B \mathcal{R}_{m}$. In diagram (9.2) below, we will eventually be proving that the upper right-hand corner and the lower left-hand corner are contractible (easy, and Proposition 9.13, respectively).

Proposition 9.11. The following diagram is a homotopy pushout diagram:

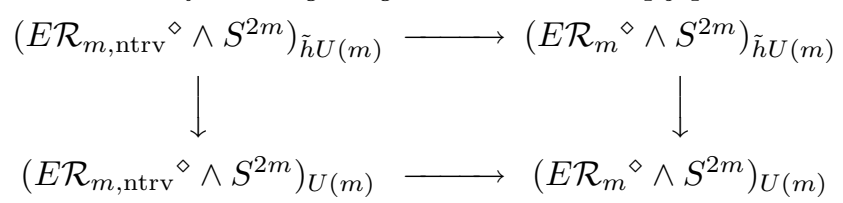

Proof. As observed in the proof of Corollary 9.10, the singular set of $E \mathcal{R}_{m}$ is $E \mathcal{R}_{m, \text { ntrv }}$. Therefore $\left(E \mathcal{R}_{m, \text { ntrv }}{ }^{\diamond} \wedge S^{2 m}\right)$ contains the singular set of $\left(E \mathcal{R}_{m} \diamond \wedge S^{2 m}\right)$, and the cofiber of the map

$$
\left(E \mathcal{R}_{m, \mathrm{ntrv}}{ }^{\diamond} \wedge S^{2 m}\right) \rightarrow\left(E \mathcal{R}_{m}^{\diamond} \wedge S^{2 m}\right)
$$

has a free action of $U(m)$ (in the based sense). It follows that orbits and homotopy orbits on this cofiber are homotopy equivalent. We conclude that the induced map 
between the horizontal cofibers in (9.2) is a homotopy equivalence, which establishes the proposition.

Our next step is to show that the orbit space $\left(E \mathcal{R}_{m, n t r v} \diamond \wedge S^{2 m}\right)_{U(m)}$ in the lower left-hand corner of (9.2) is contractible. First we look at orbit spaces for $S^{2 m}$ alone.

Lemma 9.12. If $H \in \mathcal{R}_{m, \mathrm{ntrv}}$, then $\left(S^{2 m}\right)_{H} \simeq *$.

Proof. First we note that $\left(S^{2 m}\right)_{U(m)} \simeq *$. Then consider $H=U\left(m_{1}\right) \times \cdots \times$ $U\left(m_{s}\right) \in \mathcal{R}_{m, \text { ntrv }}$, where at least one $m_{i}>0$. Then

$$
\left(S^{2 m}\right)_{H} \cong\left(S^{2 m_{1}}\right)_{U\left(m_{1}\right)} \wedge \ldots \wedge\left(S^{2 m_{s}}\right)_{U\left(m_{s}\right)} \wedge S^{2\left(m-m_{1}-\cdots-m_{s}\right)}
$$

and this establishes the result.

Now we are in a position to prove that the lower left-hand corner of (9.2) is contractible.

\section{Proposition 9.13.}

$$
\left(E \mathcal{R}_{m, \mathrm{ntrv}} \diamond \wedge S^{2 m}\right)_{U(m)} \simeq *
$$

Proof. We argue by direct analogy to [2], Lemma 7.11 and Lemma 7.12. Suppose that $X$ is a pointed $U(m)$-space whose isotropy groups are in $\mathcal{R}_{m, \text { ntrv }} \cup\{U(m)\}$. We prove that $\left(X \wedge S^{2 m}\right)_{U(m)}$ is contractible by inducting over the cells of $X$. The statement is certainly true if $X=*$. It is sufficient to show that if $\left(Y \wedge S^{2 m}\right)_{U(m)}$ is contractible and $X$ is obtained from $Y$ by adding a single $U(m)$-cell of the form $(U(m) / H) \times(\Delta[i], \partial \Delta[i])$, where $H=U\left(m_{1}\right) \times \cdots \times U\left(m_{s}\right)$ with at least one $m_{i}>0$, then $\left(X \wedge S^{2 m}\right)_{U(m)}$ is contractible. Then we have a cofiber sequence

$$
\left(Y \wedge S^{2 m}\right)_{U(m)} \rightarrow\left(X \wedge S^{2 m}\right)_{U(m)} \rightarrow S^{i} \wedge\left(S^{2 m}\right)_{H}
$$

and the first and third terms are contractible.

In our goal of proving Theorem 9.4, we are now able to relate the right side of the equivalence in $(9.1)$ to $\left(E \mathcal{R}_{m} \diamond \wedge S^{2 m}\right)_{U(m)}$, a space more clearly related to $B \mathcal{R}_{m}$, which is, after all, an orbit space.

Corollary 9.14. $\Sigma\left(E \mathcal{R}_{m, n t r v} \diamond \wedge S^{2 m}\right)_{\tilde{h} U(m)} \simeq\left(E \mathcal{R}_{m} \diamond \wedge S^{2 m}\right)_{U(m)}$.

Proof. Applying Proposition 9.13 to (9.2) tells us that the lower left-hand corner of (9.2) is contractible. On the other hand, $\mathcal{R}_{m}$ contains the trivial subgroup, so $E \mathcal{R}_{m}$ is contractible, which makes the upper right-hand corner of (9.2) contractible.

We are now in a position to give the proof of Theorem 9.4.

Proof of Theorem 9.4. We must establish that

$$
\Sigma B \mathcal{R}_{m} \simeq \Sigma\left(E \mathcal{L}_{m}^{\diamond} \wedge S^{2 m}\right)_{\tilde{h} U(m)}
$$

and then the other equivalence of the theorem follows from the equivariant weak equivalence $E \mathcal{L}_{m} \rightarrow\left|\mathcal{L}_{m}\right|$. We know from (9.1) that

$$
\Sigma\left(E \mathcal{L}_{m}^{\diamond} \wedge S^{2 m}\right)_{\tilde{h} U(m)} \simeq \Sigma\left(E \mathcal{R}_{m, \mathrm{ntrv}}{ }^{\diamond} \wedge S^{2 m}\right)_{\tilde{h} U(m)} .
$$

By Corollary 9.14,

$$
\Sigma\left(E \mathcal{R}_{m, \mathrm{ntrv}} \diamond \wedge S^{2 m}\right)_{\tilde{h} U(m)} \simeq\left(E \mathcal{R}_{m} \diamond \wedge S^{2 m}\right)_{U(m)} .
$$


We must pick out $\Sigma B \mathcal{R}_{m}$ from $\left(E \mathcal{R}_{m} \diamond \wedge S^{2 m}\right)_{U(m)}$.

The main calculation is to prove that the inclusion $S^{0} \rightarrow S^{2 m}$ induces a $U(m)$ equivalence

$$
\left(E \mathcal{R}_{m}^{\diamond} \wedge S^{0}\right) \rightarrow\left(E \mathcal{R}_{m}^{\diamond} \wedge S^{2 m}\right)
$$

We argue as in [2], Lemma 7.12. By Proposition 9.9, we know that $E \mathcal{R}_{m} \rightarrow E \mathcal{G}_{m}$ is a $U(m)$-equivalence, so it suffices to prove that

$$
\left(E \mathcal{G}_{m} \diamond \wedge S^{0}\right) \rightarrow\left(E \mathcal{G}_{m}^{\diamond} \wedge S^{2 m}\right)
$$

is a $U(m)$-equivalence. For this, we check the fixed-point sets

$$
\left(E \mathcal{G}_{m} \diamond \wedge S^{0}\right)^{H} \rightarrow\left(E \mathcal{G}_{m}^{\diamond} \wedge S^{2 m}\right)^{H}
$$

for all subgroups $H \subseteq U(m)$. There are two cases. If $\operatorname{Fix}_{H}\left(\mathbb{C}^{m}\right)=0$, then $\left(S^{2 m}\right)^{H}=$ $S^{0}$, because $H$ also fixes the point at infinity. Thus for these subgroups $H$, the map on fixed-point sets will be an equivalence because of what happens on the spheres. On the other hand, if $H$ acts on $\mathbb{C}^{m}$ with $\operatorname{Fix}_{H}\left(\mathbb{C}^{m}\right) \neq 0$, then $H$ must be a good subgroup, and so $\left(E \mathcal{G}_{m}\right)^{H} \simeq *$. In this case, the map of fixed-point sets is a map between contractible spaces.

Because (9.3) is a $U(m)$-equivalence, there is an equivalence of orbit spaces

$$
\left(E \mathcal{R}_{m} \diamond \wedge S^{0}\right)_{U(m)} \rightarrow\left(E \mathcal{R}_{m} \diamond \wedge S^{2 m}\right)_{U(m)} .
$$

But $\left(E \mathcal{R}_{m} \diamond \wedge S^{0}\right)_{U(m)}$ is $\Sigma B \mathcal{R}_{m}$, which finishes the proof.

We close the section with the proofs of Propositions 9.8 and 9.9. We will need the following characterizations of "standard" and "complete" in those proofs.

\section{Lemma 9.15.}

(1) A subgroup $H \subseteq U(m)$ is standard iff for every $H$-irreducible subspace $V \subseteq \mathbb{C}^{m}$, either $U(V) \subseteq H$ or $U(V) \cap H=\{e\}$.

(2) A subgroup $H \subseteq U(m)$ is complete iff for every $H$-irreducible subspace $V \subseteq \mathbb{C}^{m}$, we have $U(V) \subseteq H$.

Proof of Proposition 9.8. We need to check that the map is a homotopy equivalence on the fixed-point sets:

$$
\left(E \mathcal{L}_{m}\right)^{H} \rightarrow\left(E \mathcal{R}_{m, \text { ntrv }}\right)^{H} .
$$

By Lemma 2.4 of [2], it is sufficient to check this for $H \in \mathcal{R}_{m, \text { ntrv }}$ (because $\mathcal{L}_{m} \subseteq$ $\left.\mathcal{R}_{m, \text { ntrv }}\right)$. The characteristic property of the universal space for a collection means that if $H \in \mathcal{R}_{m, \mathrm{ntrv}}$, then $\left(E \mathcal{R}_{m, \mathrm{ntrv}}\right)^{H}$ is contractible, so we must show that $\left(E \mathcal{L}_{m}\right)^{H} \simeq *$. Let $H \downarrow \mathcal{L}_{m}$ denote the poset of subgroups $\left\{K \in \mathcal{L}_{m} \mid H \subseteq K\right\}$. By [5], Lemma 2.14, we know that $\left(E \mathcal{L}_{m}\right)^{H}$ is homotopy equivalent to $\left|H \downarrow \mathcal{L}_{m}\right|$.

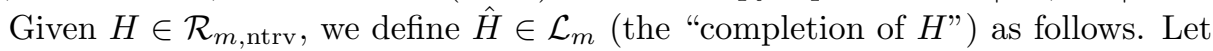
$V=\operatorname{Fix}_{H}\left(\mathbb{C}^{m}\right)$, and note that $H \subseteq U\left(V^{\perp}\right)$. Because $H$ is nontrivial, we know that $V \neq \mathbb{C}^{m}$. Let $\hat{H}=H \times U(V) \in\left(H \downarrow \mathcal{L}_{m}\right)$. Thus $\hat{H} \subseteq U\left(V^{\perp}\right) \times U(V)$.

We assert that there is a right adjoint to the inclusion functor between the posets

$$
\left(H \downarrow \mathcal{L}_{m}\right) \cap\left(\mathcal{L}_{m} \downarrow \hat{H}\right) \rightleftarrows\left(H \downarrow \mathcal{L}_{m}\right) .
$$

The retraction is given by taking $K \in\left(H \downarrow \mathcal{L}_{m}\right)$ to $K \cap \hat{H}$, which we claim is an element of $\left(\mathcal{L}_{m} \downarrow \hat{H}\right)$. Certainly $K \cap \hat{H} \subseteq \hat{H}$, and the only question is whether $K \cap \hat{H}$ is complete, i.e., an object of $\mathcal{L}_{m}$. 
Note that the isotypical summands of $\hat{H}$ are the same as those of $H$, and the only difference is that if $H$ is not complete, then $\hat{H}$, which is complete, turns $\operatorname{Fix}_{H}\left(\mathbb{C}^{m}\right)$ into a single nontrivial irreducible summand of $\hat{H}$. To check that $K \cap \hat{H}$ is complete, we use Lemma $9.15(2)$. Suppose that $W$ is a $(K \cap \hat{H})$-irreducible subspace. Note that since $H \subseteq K \cap \hat{H} \subseteq \hat{H}$, then $W$ is contained in an $\hat{H}$-irreducible, which means that either $W$ is actually a nontrivial $H$-irreducible or $W \subseteq \operatorname{Fix}_{H}\left(\mathbb{C}^{m}\right)$.

- If $W$ is a nontrivial $H$-irreducible, then $U(W) \subseteq H$ since $H$ is standard, and thus $U(W) \subseteq K \cap \hat{H}$.

- If $W \subseteq \operatorname{Fix}_{H}\left(\mathbb{C}^{m}\right)$, then $U(W) \subseteq \hat{H}$ by definition of $\hat{H}$. Further, since $K \cap \hat{H} \subseteq K$, we must have $W \subseteq W^{\prime}$ for an irreducible $K$-module $W^{\prime}$, and so $\bar{U}(W) \subseteq U\left(W^{\prime}\right) \subseteq K$ because $K$ is complete. Thus again we have $U(W) \subseteq K \cap \hat{\hat{H}}$

Thus $K \cap \hat{H}$ is complete.

But now we note that $\left(H \downarrow \mathcal{L}_{m}\right) \cap\left(\mathcal{L}_{m} \downarrow \hat{H}\right)$ has a terminal object, namely $\hat{H}$. Thus $\left(E \mathcal{L}_{m}\right)^{H} \simeq\left|H \downarrow \mathcal{L}_{m}\right| \simeq\left|\left(H \downarrow \mathcal{L}_{m}\right) \cap\left(\mathcal{L}_{m} \downarrow \hat{H}\right)\right| \simeq *$, which is what we needed to establish.

Remark 9.16. It is necessary to have $H$ nontrivial in the proof of Proposition 9.8. If $H$ happens to be trivial, then the proof has $H=\{e\}, \hat{H}=U(m),\left(H \downarrow \mathcal{L}_{m}\right)=$ $\mathcal{L}_{m}$, and $\left(\mathcal{L}_{m} \downarrow \hat{H}\right)=\mathcal{L}_{m}$. The problem is that $\left(\mathcal{L}_{m} \downarrow \hat{H}\right)=\mathcal{L}_{m}$ no longer has $\hat{H}$ as a terminal object, because $\hat{H}=U(m) \notin \mathcal{L}_{m}$.

Proof of Proposition 9.9. We need to check that the map is a homotopy equivalence on the fixed-point sets:

$$
\left(E \mathcal{R}_{m}\right)^{H} \rightarrow\left(E \mathcal{G}_{m}\right)^{H}
$$

As in the preceding proof, it is sufficient to check that if $H \in \mathcal{G}_{m}$, then $\left(E \mathcal{R}_{m}\right)^{H} \simeq *$, i.e., that $\left|H \downarrow \mathcal{R}_{m}\right| \simeq *$.

Given $H \in \mathcal{G}_{m}$, a "good" subgroup, we define $\hat{H} \in \mathcal{R}_{m}$ as follows. Either $H$ is trivial, or the subgroup $H$ acts on $\mathbb{C}^{m}$ with more than one isotypical summand by definition, so the isotypical summands of $H$ give a decomposition $\mathbb{C}^{m} \cong V_{0} \oplus V_{1} \oplus$ $\cdots \oplus V_{k}$, where $V_{0}$ is the summand with a trivial action of $H$, and either $V_{0} \neq 0$ or $k>1$. Let $\hat{H}=\prod_{i=1}^{k} U\left(V_{i}\right)$. If $V_{0} \neq 0$, then certainly $\hat{H}$ is a proper subgroup of $U(m)$, and if $V_{0}=0$, then $k>1$, and so again $\hat{H}$ is a proper subgroup of $U(m)$. In both cases, $\hat{H}$ is in $\mathcal{R}_{m}$.

We assert that are adjoint functors between the posets

$$
\left(H \downarrow \mathcal{R}_{m}\right) \cap\left(\mathcal{R}_{m} \downarrow \hat{H}\right) \rightleftarrows\left(H \downarrow \mathcal{R}_{m}\right),
$$

where the retraction is given by $K \mapsto K \cap \hat{H}$. What is at issue is whether $K \cap \hat{H}$ is in $\mathcal{R}_{m}$, i.e., whether $K \cap \hat{H}$ is standard. The input is that $K$ is standard and $H \subseteq K$.

We will use Lemma 9.15 (1). Suppose that $V$ is a $(K \cap \hat{H})$-irreducible subspace of $\mathbb{C}^{m}$. Because $K \cap \hat{H} \subseteq \hat{H}$, the subspace $V$ is contained in an $\hat{H}$-irreducible subspace $W$, and because $\hat{H}$ is standard, either $U(W) \subseteq \hat{H}$ or $U(W) \cap \hat{H}=\{e\}$. By a similar argument, either $U(W) \subseteq K$ or $U(W) \cap K=\{e\}$. Putting these together, we find that either $U(W) \subseteq(K \cap \hat{H})$ or $U(W) \cap(K \cap \hat{H})=\{e\}$, and so $K \cap \hat{H}$ is standard. 
But $\hat{H}$, being standard, is an element of $\mathcal{R}_{m}$, and thus $\left(H \downarrow \mathcal{R}_{m}\right) \cap\left(\mathcal{R}_{m} \downarrow \hat{H}\right)$ has $\hat{H}$ as a terminal object. Therefore $\left|\left(H \downarrow \mathcal{R}_{m}\right) \cap\left(\mathcal{R}_{m} \downarrow \hat{H}\right)\right|$ is contractible, and so is $\left|H \downarrow \mathcal{R}_{m}\right|$. Thus $\left(E \mathcal{R}_{m}\right)^{H} \simeq *$ for $H \in \mathcal{G}_{m}$, which finishes the proof of the proposition.

\section{Partition posets, Tits Buildings And DUality, Revisited}

Our main goal in this section is to prove the following theorem, whose proof is found at the end of the section. This is a shifted self-duality result for the complexes $\left|\mathcal{L}_{p^{k}}\right|^{\diamond}$ of direct-sum decompositions introduced in Section 9. In view of the description in [2], 1.16, we can see that Corollary 10.2 is analogous to [2], Theorem 1.17.

Theorem 10.1. Let $W$ be a spectrum with an action of $U\left(p^{k}\right)$. There is a $U\left(p^{k}\right)$ equivariant map that is a $\bmod p$ equivalence

$$
S^{-k+1} \wedge\left|\mathcal{L}_{p^{k}}\right|^{\diamond} \wedge W \simeq S^{k} \wedge \operatorname{map}\left(\left|\mathcal{L}_{p^{k}}\right|^{\triangleright}, W \wedge S^{\operatorname{ad}_{p^{k}}}\right),
$$

where $S^{\mathrm{ad}_{p^{k}}}$ is the one-point compactification of the adjoint representation of $U\left(p^{k}\right)$.

Corollary 10.2. There is a mod $p$ equivalence of homotopy orbit spectra

$$
S^{-k+1} \wedge\left(\left|\mathcal{L}_{p^{k}}\right|^{\diamond} \wedge W\right)_{\tilde{h} U\left(p^{k}\right)} \simeq S^{k} \wedge \operatorname{map}\left(\left|\mathcal{L}_{p^{k}}\right|^{\diamond}, W \wedge S^{\operatorname{ad}_{p^{k}}}\right)_{\tilde{h} U\left(p^{k}\right)} .
$$

We will also need the following result in Section 11, and we give its proof at the very end of the section. The group $\Gamma_{k}$ is described below in (10.1).

Proposition 10.3. The homotopy orbit spectra of Corollary 10.2 are wedge summands of $S^{1} \wedge W_{\tilde{h} \Gamma_{k}}$ (after p-completion).

The proof of Theorem 10.1, which is the penultimate item in the section, is closely parallel to Sections 8 and 9 of [2]. In particular, we approximate both sides of the desired equivalence using an appropriate (symplectic) Tits building (Proposition 10.6 and Corollary 10.9), and then show that the Tits building in question has a self-duality property (Proposition 10.10) that gives a bridge between the two.

We begin by discussing the group-theoretic aspects. A crucial ingredient of [2] is the subgroup $\Delta_{k} \subset \Sigma_{p^{k}}$, which is characterized uniquely up to conjugacy by being a transitive elementary abelian $p$-subgroup of $\Sigma_{p^{k}}$. The subgroup $\Delta_{k}$ can be thought of as $\left(\mathbf{F}_{p}\right)^{k}$ acting on itself by translation to give a transitive subgroup of the permutation group of $p^{k}$ objects. The normalizer $N \Delta_{k}$ can be identified with the affine group $\operatorname{Aff}_{k}\left(\mathbf{F}_{p}\right)$, and the Weyl group $\left(N \Delta_{k}\right) / \Delta_{k}$ is $\mathrm{GL}_{k}\left(\mathbf{F}_{p}\right)$, so we have a short exact sequence

$$
1 \rightarrow \Delta_{k} \rightarrow \operatorname{Aff}_{k}\left(\mathbf{F}_{p}\right) \rightarrow \mathrm{GL}_{k}\left(\mathbf{F}_{p}\right) \rightarrow 1 .
$$

The analogous subgroup of the unitary group is $\Gamma_{k} \subset U\left(p^{k}\right)$. It is described in detail in [15] (see also [1], p. 464), but we give a brief description here. The subgroup $\Gamma_{k}$ is a central extension of $(\mathbb{Z} / p)^{2 k}$ by the circle group $S^{1}$ that is the center of $U\left(p^{k}\right)$ :

$$
1 \rightarrow S^{1} \rightarrow \Gamma_{k} \rightarrow(\mathbb{Z} / p)^{2 k} \rightarrow 1 .
$$

The realization by matrices of the quotient $\Gamma_{k} / S^{1} \cong(\mathbb{Z} / p)^{2 k}$ uses permutation matrices for one of the copies of $(\mathbb{Z} / p)^{k}$, via the inclusion $\Delta_{k} \cong(\mathbb{Z} / p)^{k} \hookrightarrow \Sigma_{p^{k}} \hookrightarrow$ 
$U\left(p^{k}\right)$, while the other copy of $(\mathbb{Z} / p)^{k}$ is realized by certain diagonal matrices. The subgroup $\Gamma_{k}$ is irreducible, corresponding to the fact that $\Delta_{k}$ is transitive. Let $A_{k}$ be the normalizer of $\Gamma_{k}$ in $U\left(p^{k}\right)$. There is a short exact sequence

$$
1 \rightarrow \Gamma_{k} \rightarrow A_{k} \rightarrow \operatorname{Sp}_{2 k}\left(\mathbf{F}_{p}\right) \rightarrow 1
$$

where $\operatorname{Sp}_{2 k}\left(\mathbf{F}_{p}\right)$ is the symplectic group, which is therefore the Weyl group of $\Gamma_{k}$ in $U\left(p^{k}\right)$.

The subgroup $\Gamma_{k}$ also has a uniqueness property analogous to that of $\Delta_{k}$. We follow the terminology of [1] and call a subgroup of $U(m)$ a "projective elementary abelian $p$-subgroup" if it is the extension of an elementary abelian $p$-subgroup of $U(m)$ by $S^{1}=Z(U(m))$. Then it is a standard fact from projective representation theory that $\Gamma_{k}$ is, up to conjugacy, the unique irreducible projective elementary abelian $p$-subgroup of $U\left(p^{k}\right)$ (e.g., see [22]). In fact, just as $\Sigma_{m}$ has no transitive elementary abelian $p$-subgroups unless $m=p^{k}$ for some $k$, so $U(m)$ has no irreducible projective elementary abelian $p$-subgroups unless $m=p^{k}$.

The next step is to introduce the symplectic Tits building. Because $\left[\Gamma_{k}, \Gamma_{k}\right]=$ $\mathbb{Z} / p \subset S^{1}=Z\left(\Gamma_{k}\right)$, the commutator defines a skew-symmetric bilinear map [-, - ] : $\Gamma_{k} \times \Gamma_{k} \rightarrow \mathbb{Z} / p$. Moreover, since the connected component of the identity is the center, it follows that the commutator passes to a skew-symmetric bilinear form on

$$
\pi_{0}\left(\Gamma_{k}\right) \cong \Gamma_{k} / S^{1} \cong \mathbf{F}_{p}^{2 k}
$$

The form is clearly non-degenerate, and therefore is a symplectic form.

Recall that a subspace $V$ of a symplectic space is called coisotropic if $V^{\perp} \subset V$. Let us say that a subgroup of $\Gamma_{k}$ is "coisotropic" if it is the inverse image of a coisotropic subspace of $\mathbf{F}_{p}^{2 k}$ under the projection $\Gamma_{k} \rightarrow \mathbf{F}_{p}^{2 k}$. Let $\mathrm{TSp}_{2 k}$ denote the collection of proper coisotropic subgroups of $\Gamma_{k}$. We define the Tits building for the symplectic group $\operatorname{Sp}_{2 k}\left(\mathbf{F}_{p}\right)$ to be $\left|\operatorname{TSp}_{2 k}\right|^{\diamond}$, which turns out to have the homotopy type of a wedge of spheres of dimension $k$.

To relate the symplectic Tits building to the constructions of Section 9 , we prove the following lemma.

Lemma 10.4. A proper coisotropic subgroup of $\Gamma_{k}$ is a good subgroup of $U\left(p^{k}\right)$, in the sense of Definition 9.1.

Proof. Let $H$ be a proper coisotropic subgroup of $\Gamma_{k}$, and suppose that $H$ is bad. Then the restriction to $H$ of the standard representation of $U\left(p^{k}\right)$ on $\mathbb{C}^{p^{k}}$ is of the form $V \otimes \mathbb{C}^{s}$, where $V$ is a nontrivial irreducible representation of $H$ and $\mathbb{C}^{s}$ is the trivial $s$-dimensional representation. It follows that $\operatorname{dim}(V) \mid p^{k}, \operatorname{so} \operatorname{dim}(V)=p^{l}$ for some $l \leq k$. Moreover, since $H$ acts faithfully on $V \otimes \mathbb{C}^{s}$, it follows that $H$ acts faithfully on $V$, and so $H$ is an irreducible subgroup of $U\left(p^{l}\right)$. Since $H$ is also a projective elementary abelian $p$-subgroup, it follows that $H \cong \Gamma_{l}$. On the other hand, the assumption that $H$ is a proper coisotropic group of $\Gamma_{k}$ implies that $\pi_{0}(H)^{\perp}$ is a nontrivial subgroup of $\pi_{0}(H)$. But then $H$ cannot be isomorphic to $\Gamma_{l}$, because $\pi_{0}\left(\Gamma_{l}\right)^{\perp}=\{0\}$, and we reach a contradiction.

Recall that $\mathcal{G}_{p^{k} \text {,ntrv }}$ denotes the collection of all nontrivial good subgroups of $U\left(p^{k}\right)$. The inclusion of collections $\mathrm{TSp}_{2 k} \hookrightarrow \mathcal{G}_{p^{k}, \text { ntrv }}$ is equivariant with respect to the group inclusion $A_{k} \hookrightarrow U\left(p^{k}\right)$, and it passes to a based map of unreduced suspensions $E \operatorname{TSp}_{2 k}^{\diamond} \rightarrow E \mathcal{G}_{p^{k}, \text { ntrv }}^{\diamond}$. Inducing up, we get a $U\left(p^{k}\right)$-equivariant map $U\left(p^{k}\right)_{+} \wedge_{A_{k}} E \mathrm{TSp}_{2 k}^{\diamond} \rightarrow E \mathcal{G}_{p^{k}, \text { ntrv }}^{\diamond}$. The following proposition is a special case of 
[1], Theorem 11, namely the case $P=Z\left(U\left(p^{k}\right)\right)=S^{1}$, which acts trivially on both $U\left(p^{k}\right)_{+} \wedge_{A_{k}} E \mathrm{TSp}_{2 k}^{\diamond}$ and $E \mathcal{G}_{p^{k}, \text { ntrv }}^{\diamond}$. Although the definition of "good subgroup" in this current work is not exactly the same as that used in [1] (where it is required that the center, $S^{1}$, be contained in a good subgroup), an argument with fixedpoint sets shows that the space $\mathrm{E}\left(F_{p^{k}}\right)$ of [1] is $U\left(p^{k}\right)$-equivariantly equivalent to $E \mathcal{G}_{p^{k}, \text { ntrv }}$

Proposition 10.5. The above $U\left(p^{k}\right)$-equivariant map is a mod $p$ homology equivalence

$$
U\left(p^{k}\right)_{+} \wedge_{A_{k}}\left(E \operatorname{TSp}_{2 k}\right)^{\diamond} \rightarrow\left(E \mathcal{G}_{p^{k}, \text { ntrv }}\right)^{\diamond}
$$

We will not reproduce the proof here, but we remark that an essential input is that all irreducible projective elementary abelian $p$-subgroups of $U\left(p^{k}\right)$ are conjugate to $\Gamma_{k}$, just as it is essential for [2] that all transitive elementary abelian $p$-subgroups of $\Sigma_{p^{k}}$ are conjugate to $\Delta_{k}$.

Thus we arrive at the following $\bmod p$ approximation result for the complex $\left|\mathcal{L}_{p^{k}}\right|^{\diamond}$ of direct-sum decompositions.

Proposition 10.6. There is a chain of $U\left(p^{k}\right)$-equivariant maps that are mod $p$ equivalences

$$
U\left(p^{k}\right)_{+} \wedge_{A_{k}}\left|\mathrm{TSp}_{2 k}\right|^{\diamond} \simeq_{p}\left|\mathcal{L}_{p^{k}}\right|^{\diamond}
$$

Proof. There is a commuting square

$$
\begin{array}{rr}
U\left(p^{k}\right)_{+} \wedge_{A_{k}}\left(E \operatorname{TSp}_{2 k}\right)^{\diamond} \stackrel{\simeq_{p}}{\longrightarrow}\left(E \mathcal{G}_{p^{k}, \text { ntrv }}\right)^{\diamond} \\
\simeq \downarrow & \simeq \downarrow \\
U\left(p^{k}\right)_{+} \wedge_{A_{k}}\left|\mathrm{TSp}_{2 k}\right|^{\diamond} \longrightarrow\left|\mathcal{G}_{p^{k}, \mathrm{ntrv}}\right|^{\diamond},
\end{array}
$$

where the top map is the mod $p$ homology equivalence of Proposition 10.5, and where the vertical maps are induced by $E \mathrm{TSp}_{2 k} \rightarrow\left|\mathrm{TSp}_{2 k}\right|$ and $E \mathcal{G}_{p^{k}, \text { ntrv }} \rightarrow$ $\left|\mathcal{G}_{p^{k}, \text { ntrv }}\right|$, which are weak equivalences of spaces and equivariant with respect to $A_{k}$ and $U\left(p^{k}\right)$, respectively, by [2], 2.10.

The proposition follows, once we establish that $\left|\mathcal{L}_{p^{k}}\right| \rightarrow\left|\mathcal{G}_{p^{k}, \text { ntrv }}\right|$ is a weak equivalence. By Proposition 9.8, the inclusion $E \mathcal{L}_{p^{k}} \rightarrow E \mathcal{R}_{p^{k}, \text { ntrv }}$ is a $U\left(p^{k}\right)$ equivalence, giving us a weak equivalence $\left|\mathcal{L}_{p^{k}}\right| \rightarrow\left|\mathcal{R}_{p^{k}, \text { ntrv }}\right|$. By Corollary 9.10, $E \mathcal{R}_{p^{k}, \text { ntrv }} \rightarrow E \mathcal{G}_{p^{k}, \text { ntrv }}$ is a $U\left(p^{k}\right)$-equivalence, which gives us a weak equivalence $\left|\mathcal{R}_{p^{k}, \text { ntrv }}\right| \rightarrow\left|\mathcal{G}_{p^{k}, \text { ntrv }}\right|$, finishing the proof.

Remark 10.7. Proposition 10.6 is implicit in [1], Corollary 12, in which there are, however, a couple of misprints. The most serious one is that in the description of the map at the end of [1], Corollary 12, the wrong adjoint sphere is indicated as the target of the map. It has to be the adjoint sphere of $A_{k}$ (which is just $S^{1}$ with the trivial action), rather than the adjoint sphere of $U\left(p^{k}\right)$. We will correct the typo in Corollary 10.9.

Proposition 10.6 has the following immediate consequence.

Corollary 10.8. Let $W$ be a spectrum with an action of $U\left(p^{k}\right)$. There is a chain of $\bmod p$ equivalences

$$
\left(\left|\operatorname{TSp}_{2 k}\right|^{\diamond} \wedge W\right)_{\tilde{h} A_{k}} \simeq\left(\left|\mathcal{L}_{p^{k}}\right|^{\diamond} \wedge W\right)_{\tilde{h} U\left(p^{k}\right)}
$$


We also obtain the following dual result, which is a correction of [1], Corollary 12.

Corollary 10.9. Let $W$ be a spectrum with an action of $U\left(p^{k}\right)$. Let $S^{\operatorname{ad}_{p^{k}}}$ be the one-point compactification of the adjoint representation of $U\left(p^{k}\right)$. There is a chain of $U\left(p^{k}\right)$-equivariant maps that are $\bmod p$ equivalences

$$
\operatorname{map}\left(\left|\mathcal{L}_{p^{k}}\right|^{\diamond}, W \wedge S^{\operatorname{ad}_{p^{k}}}\right) \simeq U\left(p^{k}\right)_{+} \wedge_{A_{k}} \operatorname{map}\left(\left|\operatorname{TSp}_{2 k}\right|^{\diamond}, W \wedge S^{1}\right) .
$$

Proof. Regarding the left-hand side of (10.3), it follows immediately from Proposition 10.6 that there is a mod $p$ equivalence via equivariant maps

$$
\operatorname{map}\left(\left|\mathcal{L}_{p^{k}}\right|^{\diamond}, W \wedge S^{\operatorname{ad}_{p^{k}}}\right) \simeq \operatorname{map}\left(U\left(p^{k}\right)_{+} \wedge_{A_{k}}\left|\mathrm{TSp}_{2 k}\right|^{\diamond}, W \wedge S^{\operatorname{ad}_{p^{k}}}\right) .
$$

To transform the right-hand side of (10.3), we need the Wirthmüller isomorphism, a standard fact in equivariant Spanier-Whitehead duality. (See, for example, [13].) Let $G$ be a compact Lie group with a closed subgroup $H$, and let $E$ be a spectrum with an action of $H$. Let $S^{G}$ and $S^{H}$ be the one-point compactifications of the adjoint representations of $G$ and $H$, respectively. We give $G_{+} \wedge\left(E \wedge S^{H}\right)$ a left action of $G \times H$ by letting letting $H$ act diagonally on $G_{+} \wedge\left(E \wedge S^{H}\right)$ and letting $G$ act on the right of $G_{+}$by the inverse. This action passes to a left action of $G$ on $G_{+} \wedge_{H}\left(E \wedge S^{H}\right)$. Similarly, we define a left action of $G \times H$ on $\operatorname{map}\left(G_{+}, E \wedge S^{G}\right)$ as follows: $H$ acts diagonally on $E \wedge S^{G}$ and by conjugation on a map $f$, and $G$ acts by precomposition by right multiplication. Thus we obtain an action of $G$ on $\operatorname{map}_{H}\left(G_{+}, E \wedge S^{G}\right)$. Then the Wirthmüller isomorphism says that there is a chain of $G$-equivariant maps that are nonequivariant equivalences

$$
G_{+} \wedge_{H}\left(E \wedge S^{H}\right) \simeq \operatorname{map}_{H}\left(G_{+}, E \wedge S^{G}\right) .
$$

We apply the Wirthmüller isomorphism with $G=U\left(p^{k}\right), H=A_{k}$, and $E=$ $\operatorname{map}\left(\left|\mathrm{TSp}_{2 k}\right|^{\diamond}, W\right)$. Note that $A_{k}$ is a 1-dimensional group with trivial adjoint representation, so the one-point compactification of the adjoint representation of $A_{k}$ is the circle with trivial action. Thus we get

$$
\begin{aligned}
U\left(p^{k}\right)_{+} \wedge_{A_{k}}\left(\operatorname { m a p } \left(\left|\mathrm{TSp}_{2 k}\right|^{\diamond},\right.\right. & \left.W) \wedge S^{1}\right) \\
& \simeq \operatorname{map}_{A_{k}}\left(U\left(p^{k}\right)_{+}, \operatorname{map}\left(\left|\mathrm{TSp}_{2 k}\right|^{\diamond}, W\right) \wedge S^{\operatorname{ad}_{p^{k}}}\right) .
\end{aligned}
$$

Finally, standard arguments in equivariant topology show that there is a $U\left(p^{k}\right)$ equivariant equivalence

$$
\begin{aligned}
\operatorname{map}\left(U\left(p^{k}\right)_{+} \wedge_{A_{k}}\left|\mathrm{TSp}_{2 k}\right|^{\diamond},\right. & \left.W \wedge S^{\operatorname{ad}_{p^{k}}}\right) \\
& \simeq \operatorname{map}_{A_{k}}\left(U\left(p^{k}\right)_{+}, \operatorname{map}\left(\left|\mathrm{TSp}_{2 k}\right|^{\diamond}, W \wedge S^{\operatorname{ad}_{p^{k}}}\right)\right),
\end{aligned}
$$

We have now established approximation results for the space of direct-sum decompositions in terms of the symplectic Tits building. The other ingredient that we need is a self-duality result for the symplectic Tits building. We follow closely the discussion in [2], Section 8, where a similar result is established for the Tits building for the general linear group. $\left|\mathrm{TSp}_{2 k}\right|^{\diamond}$ has a natural structure of a $k$-dimensional simplicial complex, with $k$-dimensional simplices identified with $\operatorname{Sp}_{2 k}\left(\mathbf{F}_{p}\right) / B$, where 
$B$ is the Borel subgroup of upper triangular symplectic matrices. It follows that there is a map of spectra

$$
\alpha: S^{-k} \wedge \Sigma^{\infty}\left|\mathrm{TSp}_{2 k}\right|^{\diamond} \rightarrow \Sigma^{\infty}\left(\operatorname{Sp}_{2 k}\left(\mathbf{F}_{p}\right) / B\right)_{+}
$$

obtained by desuspending $k$ times the map that collapses the $(k-1)$-skeleton to a point. Dualizing, we obtain the map

$$
D(\alpha): \operatorname{map}\left(\left(\operatorname{Sp}_{2 k}\left(\mathbf{F}_{p}\right) / B\right)_{+}, \mathbf{S}\right) \rightarrow S^{k} \wedge \operatorname{map}\left(\left|\mathrm{TSp}_{2 k}\right|^{\diamond}, \mathbf{S}\right) .
$$

Moreover, since $\mathrm{Sp}_{2 k}\left(\mathbf{F}_{p}\right)$ is a finite group, there is an $\mathrm{Sp}_{2 k}\left(\mathbf{F}_{p}\right)$-equivariant weak equivalence $\beta: \Sigma^{\infty}\left(\mathrm{Sp}_{2 k}\left(\mathbf{F}_{p}\right) / B\right)_{+} \rightarrow \operatorname{map}\left(\left(\operatorname{Sp}_{2 k}\left(\mathbf{F}_{p}\right) / B\right)_{+}, \mathbf{S}\right)$.

Proposition 10.10. The composition

$$
D(\alpha) \circ \beta \circ \alpha: S^{-k} \wedge \Sigma^{\infty}\left|\mathrm{TSp}_{2 k}\right|^{\diamond} \rightarrow S^{k} \wedge \operatorname{map}\left(\left|\operatorname{TSp}_{2 k}\right|^{\triangleright}, \mathbf{S}\right)
$$

is an $A_{k}$-equivariant map inducing an isomorphism in mod $p$ homology.

Proof. The proposition is analogous to [2], Theorem 8.2, where the same result is proved for the general linear group, and we sketch an entirely analogous proof. First, both spectra are homotopy equivalent to a wedge of $p^{k^{2}}$ copies of the sphere spectrum, $p^{k^{2}}$ being the rank of the symplectic Steinberg representation. On the unique nontrivial mod $p$ homology group, the map induces the homomorphism

$$
S: \mathrm{H}_{k}\left(\left|\mathrm{TSp}_{2 k}\right|^{\diamond} ; \mathbf{F}_{p}\right) \rightarrow \mathbf{F}_{p}\left[\operatorname{Sp}_{2 k}\left(\mathbf{F}_{p}\right) / B\right] \rightarrow \mathrm{H}^{k}\left(\left|\mathrm{TSp}_{2 k}\right|^{\diamond} ; \mathbf{F}_{p}\right) .
$$

This homomorphism is analogous to the homomorphism of the same name at the bottom of [2], page 249 .

We need to prove that $S$ is an isomorphism, and the proof proceeds exactly as in [2], Lemma 8.1, the only difference being that the Weyl group of the symplectic group is a reflection group of type $C_{k}$, rather than $A_{k}$. Therefore, we have $k$ idempotents $e_{1}, \ldots, e_{k}$, and the Iwahori relations are as follows:

$$
\begin{aligned}
e_{i} e_{j} & =e_{j} e_{i} & & \text { for } 2 \leq|i-j|, \\
e_{i} e_{i+1} e_{i} & =e_{i+1} e_{i} e_{i+1} & & \text { for } 1 \leq i<k-1, \\
e_{k-1} e_{k} e_{k-1} e_{k} & =e_{k} e_{k-1} e_{k} e_{k-1} . & &
\end{aligned}
$$

For $i=0, \ldots, k-1$, we define $\bar{E}_{i}$ by $\bar{E}_{i}=\bar{e}_{k-i} \bar{e}_{k-i+1} \ldots \bar{e}_{k-1} \bar{e}_{k} \bar{e}_{k-1} \ldots \bar{e}_{k-i}$, and $w$ is defined as

$$
w=\bar{E}_{k-1} \bar{E}_{k-2} \ldots \bar{E}_{0} u .
$$

$\left(\bar{E}_{k-1} \bar{E}_{k-2} \ldots \bar{E}_{0}\right.$ is the longest word in the idempotents $\bar{e}_{i}$.) The rest of the proof of [2], Lemma 8.1 remains unchanged.

We can now give the proofs of Theorem 10.1 and Proposition 10.3.

Proof of Theorem 10.1. We assert that the following is a chain of mod $p$ equivalences.

$$
\begin{aligned}
S^{-k+1} \wedge\left|\mathcal{L}_{p^{k}}\right|^{\diamond} \wedge W & \simeq S^{-k} \wedge\left|\mathcal{L}_{p^{k}}\right|^{\diamond} \wedge W \wedge S^{1} \\
& \simeq S^{-k} \wedge U\left(p^{k}\right)_{+} \wedge A_{k}\left|\operatorname{TSp}_{2 k}\right|^{\diamond} \wedge W \wedge S^{1} \\
& \simeq S^{k} \wedge U\left(p^{k}\right)_{+} \wedge A_{A_{k}} \operatorname{map}\left(\left|\mathrm{TSp}_{2 k}\right|^{\diamond}, W \wedge S^{1}\right) \\
& \simeq S^{k} \wedge \operatorname{map}\left(\left|\mathcal{L}_{p^{k}}\right|^{\diamond}, W \wedge S^{\operatorname{ad}_{p^{k}}}\right) .
\end{aligned}
$$

The second equivalence follows from Proposition 10.6, the third follows from Proposition 10.10, and the fourth follows from Corollary 10.9. 
Proof of Proposition 10.3. The proposition follows from the relationship between Tits building and the Steinberg idempotent. Let $\epsilon \in \mathbf{F}_{p}\left[\operatorname{Sp}_{2 k}\left(\mathbf{F}_{p}\right)\right]$ be the symplectic Steinberg idempotent. Such an idempotent can be used to split off a wedge summand $\epsilon E$ from the $p$-completion of any spectrum $E$ with an action of $\operatorname{Sp}_{2 k}\left(\mathbf{F}_{p}\right)$. A homology calculation using the fact that the Tits building is spherical and the Steinberg representation is projective shows that

$$
\epsilon E \simeq_{p} S^{-k} \wedge\left(\left|\mathrm{TSp}_{2 k}\right|^{\diamond} \wedge E\right)_{\tilde{h} \operatorname{Sp}_{2 k}\left(\mathbf{F}_{p}\right)}
$$

(Cf. the comments following the proof of Theorem 1.17 of [2], page 252.) So, $S^{-k} \wedge\left(\left|\mathrm{TSp}_{2 k}\right|^{\diamond} \wedge E\right)_{\tilde{h} \operatorname{Sp}_{2 k}\left(\mathbf{F}_{p}\right)}$ is a wedge summand of the $p$-completion of $E$. In particular, this holds for $E=W_{\tilde{h} \Gamma_{k}}$ where $W$ is a spectrum with $U\left(p^{k}\right)$ action, and then we apply Corollary 10.8 .

\section{Relationship With CAlculus of FunCtors}

In this section we show that there is a relationship between, on the one hand, sequences of spectra that one obtains from Construction 3.8 in the cases of finite sets and finite-dimensional complex vector spaces, and on the other hand, certain Taylor towers that arise from the calculus of functors. We prove Theorem 11.2, which says that the subquotients of our construction show up as layers in Taylor towers, and Theorem 11.3, which analyzes our filtration in the unitary case with regard to $v_{k}$-periodic homotopy.

The general references for Goodwillie's homotopy calculus and Weiss's orthogonal calculus are $[6,21]$. We need, in particular, the Goodwillie tower of the identity and the Weiss tower of $\mathrm{BU}(-)$, for which one can look at $[3,1]$. For a general discussion of the interplay between Goodwillie's calculus and periodic homotopy, including a brief discussion of the relationship of the Goodwillie tower of the identity to the symmetric power filtration, there is Kuhn's recent survey article [10].

We very briefly recall the relevant facts. The theory of Taylor towers associates to a suitable functor $F$ a tower of fibrations of functors $P_{n} F$ that converges, under favorable circumstances, to $F$. The homotopy fiber of the map $P_{n} F \rightarrow P_{n-1} F$, which we denote $D_{n} F$, is the " $n$th layer" of $F$. It is closely related to the " $n$th derivative" of $F$.

An important functor to which one may profitably apply Goodwillie's theory is the identity functor from spaces to spaces. Recall from [3] that the Goodwillie tower of the identity functor evaluated at $S^{1}$ can be written in the following form, up to $p$-completion:

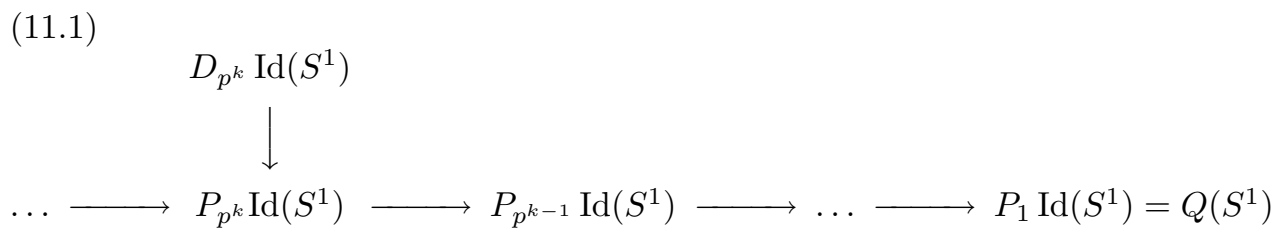

Here

$$
D_{p^{k}} \operatorname{Id}\left(S^{1}\right) \simeq \Omega^{\infty} \operatorname{map}\left(S^{1} \wedge\left|\mathcal{P}_{p^{k}}\right|^{\diamond}, \Sigma^{\infty} S^{p^{k}}\right)_{\tilde{h} \Sigma_{p^{k}}}
$$

is the homotopy fiber of the map $P_{p^{k}}\left(S^{1}\right) \rightarrow P_{p^{k-1}}\left(S^{1}\right)$, and $\mathcal{P}_{p^{k}}$ is the poset of proper, nontrivial partitions of the set $\left\{1, \ldots, p^{k}\right\}$. The tower converges to $S^{1}$ up to $p$-completion. 
Similarly, one can apply M. Weiss's theory of orthogonal calculus to the functor $V \mapsto \mathrm{BU}(V)$ from finite-dimensional complex vector spaces to topological spaces and obtain a Taylor tower for it. This tower was studied in [1], and if we take $V=\mathbb{C}$ and complete at $p$, the tower has the form

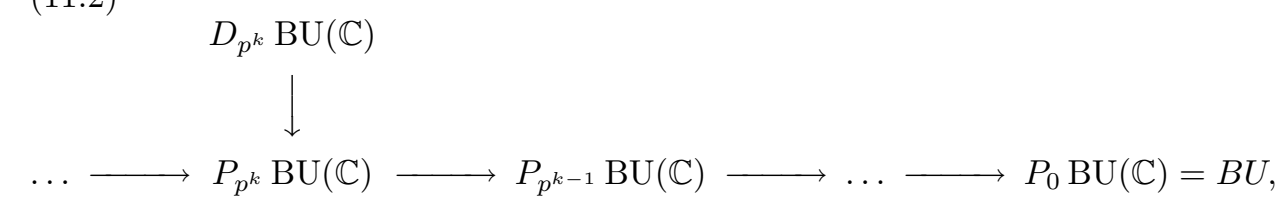

where

$$
D_{p^{k}} \mathrm{BU}(\mathbb{C}) \simeq \Omega^{\infty} \operatorname{map}\left(\left|\mathcal{L}_{p^{k}}\right|^{\diamond}, \Sigma^{\infty} S^{\mathrm{ad}_{p^{k}}} \wedge S^{2 p^{k}}\right)_{\tilde{h} U\left(p^{k}\right)}
$$

The tower converges to $B U(1)$ (up to $p$-completion).

Recall the notation

$$
\begin{aligned}
\mathrm{DI}_{k} & =D_{p^{k}} \operatorname{Id}\left(S^{1}\right) \\
\mathrm{DU}_{k} & =D_{p^{k}} \operatorname{BU}(\mathbb{C}) .
\end{aligned}
$$

We want to relate these spaces to our constructions. Our present work applied to the category of finite sets and the category of finite-dimensional complex vector spaces produces the sequences of spectra

$$
\begin{gathered}
\mathbf{S}=\operatorname{Sp}^{1}(\mathbf{S}) \rightarrow \mathrm{Sp}^{2}(\mathbf{S}) \rightarrow \cdots \rightarrow \mathrm{Sp}^{\infty}(\mathbf{S})=\mathrm{H} \mathbb{Z} \\
b u=A_{0} \rightarrow A_{1} \rightarrow A_{2} \rightarrow \cdots \rightarrow A_{\infty}=\mathrm{H} \mathbb{Z},
\end{gathered}
$$

where the quotients $\operatorname{Sp}^{m}(\mathbf{S}) / \mathrm{Sp}^{m-1}(\mathbf{S})$ and $A_{m} / A_{m-1}$ are contractible unless $m$ is a power of $p$. (The first assertion is well known, and the second is Theorem 9.7.) Moreover, if $m=p^{k}$ with $k>0$, then the homology of the above subquotients is all $p$-torsion. Therefore after $p$-completion we use the usual notation $L(k)=$ $\Sigma^{-k} \mathrm{Sp}^{p^{k}}(\mathbf{S}) / \mathrm{Sp}^{p^{k-1}}(\mathbf{S})$ for the desuspensions of quotients of symmetric powers of the sphere spectrum, which are the subquotients of our filtration applied to finite sets. Similarly, after $p$-completion we write $T(k)=\Sigma^{-(k+1)} A_{p^{k}} / A_{p^{k-1}}$ for the desuspensions of the subquotients of our filtration applied to finite-dimensional complex vector spaces and unitary isomorphisms. Before stating the main result of the section, we record the following theorem, which follows from Theorem 9.5 and Proposition 10.3 upon taking $W=\Sigma^{\infty} S^{2 p^{k}}$.

Theorem 11.1. The spectrum $T(k)$ is a wedge summand of the suspension spectrum $\Sigma^{\infty}\left(S^{2 p^{k}}\right) \tilde{h} \Gamma_{k}$.

The main result of this section is the following theorem, which says that the subquotients of our filtration show up as the layers in Taylor towers.

Theorem 11.2. For all $k \geq 0$, there are homotopy equivalences

$$
\begin{aligned}
B^{k-1} \mathrm{DI}_{k} & \simeq \Omega^{\infty} L(k) \\
B^{k-1} \mathrm{DU}_{k} & \simeq \Omega^{\infty} T(k) .
\end{aligned}
$$

Proof. The first equivalence of the theorem is a consequence of [2], Theorem 1.18. According to this theorem, there is an equivalence of infinite loop spaces

$$
B^{2 k-1} \mathrm{DI}_{k} \simeq \Omega^{\infty} \mathrm{Sp}^{p^{k}}(\mathbf{S}) / \mathrm{Sp}^{p^{k-1}}(\mathbf{S})
$$


Looping $k$ times, we find that

$$
\begin{aligned}
B^{k-1} \mathrm{DI}_{k} & \simeq \Omega^{\infty} \Sigma^{-k} \mathrm{Sp}^{p^{k}}(\mathbf{S}) / \operatorname{Sp}^{p^{k-1}}(\mathbf{S}) \\
& \simeq \Omega^{\infty} L(k),
\end{aligned}
$$

as desired.

For the second equivalence of the theorem, recall that

$$
B^{k-1} \mathrm{DU}_{k} \simeq B^{k-1} \Omega^{\infty} \operatorname{map}\left(\left|\mathcal{L}_{p^{k}}\right|^{\diamond}, \Sigma^{\infty} S^{\mathrm{ad}_{p^{k}}} \wedge S^{2 p^{k}}\right)_{\tilde{h} U\left(p^{k}\right)},
$$

and, by Theorem 9.5,

$$
A_{p^{k}} / A_{p^{k-1}} \simeq \Sigma \Sigma^{\infty}\left(\left(\left|\mathcal{L}_{p^{k}}\right|^{\diamond} \wedge S^{2 p^{k}}\right)_{\tilde{h} U\left(p^{k}\right)}\right) .
$$

Since $T(k)=\Sigma^{-(k+1)} A_{p^{k}} / A_{p^{k-1}}$,

$$
\Omega^{\infty} T(k) \simeq \Omega^{k} Q\left(\left(\left|\mathcal{L}_{p^{k}}\right|^{\diamond} \wedge S^{2 p^{k}}\right)_{\tilde{h} U\left(p^{k}\right)}\right) .
$$

The second part of the theorem now follows readily from Theorem 10.1. Indeed, applying that theorem to $W=\Sigma^{\infty} S^{2 p^{k}}$ gives the equivalence

$$
S^{k} \wedge \operatorname{map}\left(\left|\mathcal{L}_{p^{k}}\right|^{\diamond}, \Sigma^{\infty} S^{2 p^{k}} \wedge S^{\operatorname{ad}_{p^{k}}}\right) \simeq S^{-k} \wedge \Sigma^{\infty}\left|\mathcal{L}_{p^{k}}\right|^{\diamond} \wedge S^{2 p^{k}} \wedge S^{1} .
$$

Taking homotopy orbits and applying $\Omega \Omega^{\infty}$, we obtain

$$
B^{k-1} \Omega^{\infty} \operatorname{map}\left(\left|\mathcal{L}_{p^{k}}\right|^{\diamond}, \Sigma^{\infty} S^{2 p^{k}} \wedge S^{\mathrm{ad}_{p^{k}}}\right)_{\tilde{h} \mathrm{U}\left(p^{k}\right)} \simeq \Omega^{k} Q\left(\left(\left|\mathcal{L}_{p^{k}}\right|^{\diamond} \wedge S^{2 p^{k}}\right)_{\tilde{h} \mathrm{U}\left(p^{k}\right)}\right),
$$

which is the desired result.

The cohomology of map $\left(\left|\mathcal{L}_{p^{k}}\right|^{\diamond}, \Sigma^{\infty} S^{2 p^{k}} \wedge S^{\operatorname{ad}_{p^{k}}}\right)_{\tilde{h} \mathrm{U}\left(p^{k}\right)}$ was described in [1]. One consequence of Theorem 11.2 and its proof, together with [1], Theorem 4(b), is that $\mathrm{H}^{*}(T(k))$ is free over $\mathcal{A}_{k-1}$, where $\mathcal{A}_{k-1}$ is the (finite) subalgebra of the Steenrod algebra generated by $\beta, P^{1}, \ldots, P^{p^{k-1}}\left(S q^{1}, \ldots, S q^{2^{k}}\right.$ at the prime 2). Standard arguments, as in [3], then imply the following theorem.

Theorem 11.3. Let $p$ be a prime and let $K(n)$ denote the $n$th Morava $K$-theory. Then $K(n)_{*}\left(A_{p^{k}} / A_{p^{k-1}}\right)=0$ for $n<k$, and the sequence bu $=A_{0} \rightarrow A_{1} \rightarrow \mathcal{A}_{p} \rightarrow$ $A_{p^{2}} \rightarrow \cdots \rightarrow A_{\infty} \simeq \mathrm{H} \mathbb{Z}$ terminates at $A_{p^{k}}$ in $v_{k}$-periodic homotopy.

For instance, in the case $k=0$ (rational homotopy), the sequence terminates at $A_{1}$ and, rationally, $A_{1} \simeq \mathrm{H} \mathbb{Z}$. Since $A_{1} / A_{0}=\Sigma^{\infty} \Sigma \mathbb{C} P^{\infty}$ by Theorem 3.9, our sequence $\Sigma^{-1} A_{1} / A_{0} \rightarrow A_{0} \rightarrow A_{1}$ is rationally equivalent to the rational cofibration sequence

$$
\Sigma^{\infty} \mathbb{C} P^{\infty} \rightarrow b u \rightarrow \mathrm{H} \mathbb{Z}
$$

\section{Conjectures}

In this section, we make some conjectures about the more detailed relationship between our construction and Taylor towers. Roughly speaking, we conjecture that certain "chain complexes" of spectra arising from our construction are "acyclic," and that the contracting homotopy is closely related to Taylor towers. In the finite set case, the acyclicity is actually a theorem of Kuhn at the prime 2 and KuhnPriddy at odd primes [7, 9]. Informally speaking, our conjectures say, "Analogues of the theorems of Kuhn and Kuhn-Priddy hold in the unitary situation, and the structure maps of the Taylor towers deloop to contracting homotopies in both the 
case of finite sets and the case of finite-dimensional complex vector spaces." More precisely, we conjecture that certain specific maps based on stable James-Hopf maps are deloopings of the structure maps in the Taylor towers (Conjecture 12.1) and that those same maps provide contracting homotopies for the chain complexes of spectra in question (Conjecture 12.5).

We recall Kuhn's language on homological algebra of spectra [8]. A sequence of spectra

$$
E \leftarrow X_{0} \leftarrow X_{1} \leftarrow X_{2} \leftarrow \ldots
$$

is called a "complex over $E$ " if it is constructed by splicing together cofiber sequences $E_{m+1} \rightarrow X_{m} \rightarrow E_{m}$, with $E_{0}=E$. It is called a "resolution" if the differentials have sections once $\Omega^{\infty}$ is applied. It is called "projective" if the spectra $X_{m}$ are summands of suspension spectra, and it is called a "minimal" projective resolution if it is a wedge summand of any other projective resolution of $E$.

We will be using the Goodwillie tower of (11.1) and the Weiss tower of (11.2). In what follows, we refer to the two types of towers collectively as "Taylor towers" when necessary; we also assume that all spaces and spectra have been completed at the prime $p$.

To state our conjectures, we want to loop the Goodwillie tower (11.1) once and loop the Weiss tower (11.2) twice. The looped Goodwillie tower starts with $\Omega Q\left(S^{1}\right) \simeq Q\left(S^{0}\right)$ at the bottom and converges to $\Omega S^{1} \simeq \mathbb{Z}$. The twice-looped Weiss tower starts with $\Omega^{2} B U \simeq \mathbb{Z} \times B U$, by Bott periodicity, and it converges to $\Omega^{2} \mathrm{BU}(\mathbb{C}) \simeq \Omega^{2} \mathbb{C} P^{\infty} \simeq \mathbb{Z}$. In both the Goodwillie tower and the Weiss tower, we have fibration sequences of the type

$$
\Omega^{\epsilon} D_{k} \rightarrow \Omega^{\epsilon} P_{p^{k}} \rightarrow \Omega^{\epsilon} P_{p^{k-1}} \rightarrow \Omega^{\epsilon-1} D_{k},
$$

where $\epsilon$ is 1 or 2 . In both towers we have structure maps given by the composition of the inclusion of the fiber with the next $k$-invariant,

$$
\begin{aligned}
\Omega \mathrm{DI}_{k} & \longrightarrow P_{p^{k}} \mathrm{Id}\left(S^{1}\right) \longrightarrow \mathrm{DI}_{k+1} \\
\Omega^{2} \mathrm{DU}_{k} & \longrightarrow \Omega^{2} P_{p^{k}} \mathrm{BU}(\mathbb{C}) \longrightarrow \Omega \mathrm{DU}_{k+1} .
\end{aligned}
$$

It is well known that these structure maps, although maps between infinite loop spaces, are not infinite loop maps. Our first conjecture is that they are $(k+1)$ fold loop maps (Conjecture 12.1), and we will hazard a guess about the actual delooping. Our conjectural models for the delooping depend on the spaces $B^{k} \mathrm{DI}_{k}$ and $B^{k-1} \mathrm{DU}_{k}$ being retracts of certain other spaces. To begin with $\mathrm{DI}_{k}$, it is an immediate consequence of [2], Corollary 9.6, that $B^{k} \mathrm{DI}_{k}$ is a homotopy retract of the space $Q\left(S^{p^{k}}\right)_{\tilde{h} \Delta_{k}}$ where $\Delta_{k} \cong(\mathbb{Z} / p)^{k}$ is the transitive elementary abelian $p$-subgroup of $\Sigma_{p^{k}}$. We use the notation

$$
B^{k} \mathrm{DI}_{k} \stackrel{i}{\rightarrow} Q\left(S^{p^{k}}\right)_{\tilde{h} \Delta_{k}} \stackrel{r}{\rightarrow} B^{k} \mathrm{DI}_{k}
$$

for the inclusion and retraction maps. Similarly, it is an immediate consequence of Theorems 11.1 and 11.2 that after $p$-completion the space $B^{k-1} \mathrm{DU}_{k}$ is a homotopy retract of $Q\left(S^{2 p^{k}}\right)_{\tilde{h} \Gamma_{k}}$. We abuse notation and write

$$
B^{k-1} \mathrm{DU}_{k} \stackrel{i}{\rightarrow} Q\left(S^{2 p^{k}}\right)_{\tilde{h} \Gamma_{k}} \stackrel{r}{\rightarrow} B^{k-1} \mathrm{DU}_{k}
$$

for the inclusion and the retraction maps for the unitary case as well.

We need one more comment before stating the first conjecture. The group $\Sigma_{p}$ ? $\Delta_{k} \subset \Sigma_{p^{k+1}}$ contains $\Delta_{k+1}$, and the group $\Sigma_{p} \prec \Gamma_{k} \subset U\left(p^{k+1}\right)$ contains $\Gamma_{k+1}$. In 
the following conjecture, we use the term "stable James-Hopf map" to refer to the adjoint of the map $\Sigma^{\infty} Q X \rightarrow \Sigma^{\infty}\left(X^{\wedge p}\right)_{\tilde{h} \Sigma_{p}}$ that one obtains from the Snaith splitting.

Conjecture 12.1. The structure maps described in $(12.1)$ have $(k+1)$-fold deloopings. Moreover, the deloopings are given by the two compositions

$$
\begin{gathered}
F_{k}: B^{k} \mathrm{DI}_{k} \stackrel{i}{\rightarrow} Q\left(S^{p^{k}}\right)_{\tilde{h} \Delta_{k}} \stackrel{j_{p}}{\rightarrow} Q\left(S^{p^{k+1}}\right)_{\tilde{h} \Sigma_{p} \Delta_{k}} \stackrel{t r}{\longrightarrow} Q\left(S^{p^{k+1}}\right)_{\tilde{h} \Delta_{k+1}} \stackrel{r}{\rightarrow} B^{k+1} \mathrm{DI}_{k+1} \\
g_{k}: B^{k-1} \mathrm{DU}_{k} \stackrel{i}{\rightarrow} Q\left(S^{2 p^{k}}\right)_{\tilde{h} \Gamma_{k}} \stackrel{j_{p}}{\longrightarrow} Q\left(S^{2 p^{k+1}}\right)_{\tilde{h} \Sigma_{p} \Gamma_{k}} \stackrel{t r}{\longrightarrow} Q\left(S^{2 p^{k+1}}\right)_{\tilde{h} \Gamma_{k+1}} \stackrel{r}{\rightarrow} B^{k} \mathrm{DU}_{k+1}
\end{gathered}
$$
where $t r$ stands for the transfer map, $i$ and $r$ stand for the inclusion and retraction maps discussed above, and $j_{p}$ stands for the stable James-Hopf map.

Let $f_{k}=\Omega F_{k}$, and let $g_{-\infty}$ be the double loops on the first $k$-invariant in the Weiss tower (11.2). Assuming Conjecture 12.1, we may associate the following diagrams with the looped Taylor towers, where the few spaces that are not already $p$-complete need to be $p$-completed. The maps in the diagram are those defined in Conjecture 12.1, and what is conjectural is that they are deloopings of the structure maps in the Taylor towers.

Diagram 12.2.

$$
\begin{array}{cccccccc}
\mathbb{Z} & \multicolumn{1}{l}{\eta_{I}} & \Omega \mathrm{DI}_{0} & \stackrel{f_{0}}{\longrightarrow} & \mathrm{DI}_{1} & \stackrel{f_{1}}{\longrightarrow} & B \mathrm{DI}_{2} & \stackrel{f_{2}}{\longrightarrow} \ldots \\
\mathbb{Z} & \stackrel{\eta_{U}}{\longrightarrow} \mathbb{Z} \times B U \stackrel{g_{-\infty}}{\longrightarrow} & \Omega \mathrm{DU}_{0} & \stackrel{g_{0}}{\longrightarrow} & \mathrm{DU}_{1} & \stackrel{g_{1}}{\longrightarrow} & B \mathrm{DU}_{2} & \stackrel{g_{2}}{\longrightarrow} \ldots
\end{array}
$$

Here we have $\Omega \mathrm{DI}_{0}=Q S^{0}, \mathrm{DI}_{1}=Q B \Sigma_{p}$ and $\Omega \mathrm{DU}_{0}=Q \mathbb{C} P^{\infty}$. The map $f_{0}$ : $Q S^{0} \rightarrow Q B \Sigma_{p}$ is the stable James-Hopf map (also called the Kahn-Priddy map). In keeping with our exponential indexing, we think of the space $B U=D_{0} \mathrm{BU}(\mathbb{C})$ as $\mathrm{DU}_{-\infty}$ on the grounds that $p^{-\infty}=0$. This quirk of grading does not occur in the case of the Goodwillie tower, because the Goodwillie tower of the identity does not have a degree zero term. We conjecture that the map $g_{-\infty}: \mathbb{Z} \times B U \rightarrow Q \mathbb{C} P^{\infty}$, which is the double loops on the first $k$-invariant in the Weiss tower, is in fact closely related to the Becker-Gottlieb transfer ${ }^{3}$, as considered in [4]. The map $\eta_{I}$ is obtained from the suspension map $S^{1} \rightarrow Q S^{1}$ by looping down once. Similarly, the map $\eta_{U}$ is obtained from the inclusion $B U(1) \rightarrow B U$ by looping down twice. Note that in the case of the Goodwillie tower, we did not use the full strength of Conjecture 12.1. The number of deloopings that we need to string together the $k$ invariants is actually one less than provided by the conjecture, so we use $f_{k}$ rather than $F_{k}$.

On the other hand, instead of looking at the filtration sequences in the Taylor towers, we can consider the cofiber sequences of $p$-completed spectra

$$
\begin{aligned}
S p^{p^{k-1}}(\mathbf{S}) & \longrightarrow S p^{p^{k}}(\mathbf{S}) \longrightarrow \Sigma^{k} L(k) \\
A_{p^{k-1}} & \longrightarrow A_{p^{k}} \longrightarrow \Sigma^{k+1} T(k) .
\end{aligned}
$$

By splicing together the cofiber sequences for different values of $k$, the $p$-completion of the symmetric power filtration and its bu-analogue can be written in the following chain complex form.

\footnotetext{
${ }^{3}$ The referee suggests that one can also get a presumably relevant map by considering the composite $U \rightarrow Q_{S^{1}}\left(S^{0}\right) \rightarrow Q\left(\Sigma \mathbb{C} P^{\infty}\right)_{+}$, where the first map is the equivariant $J$-homomorphism and the second map is obtained from the Segal-tom Dieck splitting.
} 


\section{Diagram 12.3.}

$$
\begin{aligned}
& \mathrm{H} \mathbb{Z} \longleftarrow \epsilon_{I} \quad L(0) \stackrel{\alpha_{0}}{\longleftarrow} L(1) \stackrel{\alpha_{1}}{\longleftarrow} L(2) \stackrel{\alpha_{2}}{\longleftarrow} \ldots \\
& \mathrm{H} \mathbb{Z} \stackrel{\epsilon_{U}}{\longleftarrow} b u \stackrel{\beta_{-\infty}}{\longleftarrow} T(0) \stackrel{\beta_{0}}{\longleftarrow} T(1) \stackrel{\beta_{1}}{\longleftarrow} T(2) \stackrel{\beta_{2}}{\longleftarrow} \ldots
\end{aligned}
$$

Recall that $L(0)=\mathbf{S}, L(1)=\Sigma^{\infty} B \Sigma_{p}$ and $T(0)=\Sigma^{\infty} \mathbb{C} P^{\infty}$, where the latter follows from either Construction 3.8 itself or from Corollary 8.3.

Theorem 11.2 says that $\Omega^{\infty}$ applied to the objects in Diagram 12.3 gives the objects found in Diagram 12.2, but the maps are pointing in opposite directions. In view of this, we may combine Diagrams 12.2 and 12.3 into one as follows.

\section{Diagram 12.4.}

$$
\begin{aligned}
& \mathbb{Z} \underset{\epsilon_{I}}{\stackrel{\eta_{I}}{\rightleftarrows}} \Omega \mathrm{DI}_{0} \underset{\alpha_{0}}{\stackrel{f_{0}}{\rightleftarrows}} \mathrm{DI}_{1} \underset{\alpha_{1}}{\stackrel{f_{1}}{\rightleftarrows}} B \mathrm{DI}_{2} \underset{\alpha_{2}}{\stackrel{f_{2}}{\rightleftarrows}} \ldots \\
& \mathbb{Z} \underset{\epsilon_{U}}{\stackrel{\eta_{U}}{\rightleftarrows}} \mathbb{Z} \times \mathrm{BU} \underset{\beta_{-\infty}}{\stackrel{g_{-\infty}}{\rightleftarrows}} \Omega \mathrm{DU}_{0} \underset{\beta_{0}}{\stackrel{g_{0}}{\rightleftarrows}} \mathrm{DU}_{1} \underset{\beta_{1}}{\stackrel{g_{1}}{\rightleftarrows}} B \mathrm{DU}_{2} \underset{\beta_{2}}{\stackrel{g_{2}}{\rightleftarrows}} \ldots
\end{aligned}
$$

Strictly speaking, the maps pointing to the left are infinite loops of the maps of the same names in Diagram 12.3, but for reasons of typesetting, we omit $\Omega^{\infty}$ from the notation. Thus, the maps pointing to the left are infinite loop maps, and since they have null-homotopic composites, they constitute chain complex differentials. The maps pointing to the right are not infinite loop maps, and we do not know if their composites are null-homotopic.

It is reasonable to ask if Diagram 12.4 represents a chain complex with a contracting homotopy, and this is our main conjecture.

\section{Conjecture 12.5.}

(1) For all $k \geq 1$, the following maps are weak homotopy equivalences.

(a) $\eta_{I} \circ \epsilon_{I}+\alpha_{0} \circ f_{0}: \Omega \mathrm{DI}_{0} \rightarrow \Omega \mathrm{DI}_{0}$

(b) $f_{k-1} \circ \alpha_{k-1}+\alpha_{k} \circ f_{k}: B^{k-1} \mathrm{DI}_{k} \rightarrow B^{k-1} \mathrm{DI}_{k}$

(2) Similarly, the following maps are weak equivalences.

(a) $\eta_{U} \circ \epsilon_{U}+\beta_{-\infty} \circ g_{-\infty}: \mathbb{Z} \times B U \rightarrow \mathbb{Z} \times B U$

(b) $g_{-\infty} \circ \beta_{-\infty}+\beta_{0} \circ g_{0}: \Omega \mathrm{DU}_{0} \rightarrow \Omega \mathrm{DU}_{0}$

(c) $g_{k-1} \circ \beta_{k-1}+\beta_{k} \circ g_{k}: B^{k-1} \mathrm{DU}_{k} \rightarrow B^{k-1} \mathrm{DU}_{k}$

Finally, the compositions $\epsilon_{I} \circ \eta_{I}$ and $\epsilon_{U} \circ \eta_{U}$ give the identity map on $\mathbb{Z}$.

It is in fact easy to check that $\epsilon_{I} \circ \eta_{I}$ gives the identity map on $\mathbb{Z}$. The first interesting instance of Conjecture 12.5 in the homotopy case is (1a). It is equivalent to the assertion that the composed map $Q_{0}\left(S^{0}\right) \stackrel{j_{p}}{\longrightarrow} Q B \Sigma_{p} \stackrel{t r}{\longrightarrow} Q_{0}\left(S^{0}\right)$ is a homotopy equivalence after $p$-completion, which is the Kahn-Priddy theorem.

Likewise, it is easy to check that $\epsilon_{U} \circ \eta_{U}$ is the identity on $\mathbb{Z}$, and the first interesting instance of Conjecture 12.5 in the unitary case is (2a). It is equivalent to the assertion that the composed map $B U \stackrel{g_{-\infty}}{\longrightarrow} Q \mathbb{C} P^{\infty} \stackrel{t r}{\longrightarrow} B U$ is an equivalence. Strong evidence for this assertion is given by a theorem of Segal that says that the space $B U$ is a retract of $Q \mathbb{C} P^{\infty}$ [18]. See also [4], where Segal's theorem is reproved and generalized using the Becker-Gottlieb transfer $B U \rightarrow Q \mathbb{C} P^{\infty}$. This transfer map is probably more directly pertinent to our constructions than Segal's proof. (Or perhaps, as the referee suggests, the equivariant $J$-homomorphisms is even 
more relevant here.) To prove (2a), one only needs to show that our map $g_{-\infty}$ is close enough to the Becker-Gottlieb transfer.

Conjecture 12.5 taken together with Theorem 11.1 would imply that the two chain complexes of spectra in Diagram 12.3 are, in Kuhn's language, projective resolutions of $\mathrm{H} \mathbb{Z}$ and of $b u\langle 0\rangle$ (the fiber of the map $b u \rightarrow \mathrm{H} \mathbb{Z}$ ), respectively. In the case of $H \mathbb{Z}$, this consequence is the main theorem of [7] and [9]. In fact, it appears likely that [7] and [9] contain a proof of Conjecture 12.5 (1), or come close. One needs to check that certain maps considered there are equivalent to our maps $f_{k}$, and a superficial inspection of all the constructions involved suggests that this is the case. Thus the interesting case is Conjecture 12.5 (2).

It is also shown in [8] that the projective resolution of $\mathrm{H} \mathbb{Z}$ that one obtains from the symmetric power filtration is minimal. We conjecture that the same is true of the resolution that Conjecture 12.5 would provide for $b u\langle 0\rangle$. If true, this would answer one of the open questions proposed at the end of [8].

Conjecture 12.6. The second chain complex in Diagram 12.3 gives rise to a minimal projective resolution of bu $\langle 0\rangle$.

It is conceivable that one can prove Conjecture 12.5 (2) by carefully transferring the methods of Kuhn and Priddy to the unitary context, since all of the crucial ingredients of their proof have analogues in the present context: stable JamesHopf maps, transfer maps and a suitable family of Steinberg idempotents seem to be arranged in a similar constellation. Possibly this is the "right" way to prove the conjecture. However, we also wonder if the presence of the Taylor towersan ingredient not available at the time that $[7,9]$ were written - can be profitably incorporated into the proof, thereby making it simpler or at least in some way more "conceptual." For example, a more conceptual proof might be one that does not rely on explicit homology calculations as heavily as Kuhn's original proof.

It is intriguing to ask whether the special phenomena occurring in the cases of finite sets and complex vector spaces hold in other cases, but if they do, then it may not be in an obvious way. For example, consider the category of finitedimensional real vector spaces. Our construction associates with it a sequence of spectra interpolating between bo and $\mathrm{H} \mathbb{Z}$, and Theorems 9.5, 9.7 and 11.3 will have appropriate analogues, although the statements are likely to be a little less clean than in the complex case. Moreover, there is a Weiss tower associated with the functor $V \mapsto B O(V)$, which appears to be related to our filtration. But there may be no real-case analogue of Theorem 11.2 to establish a precise relationship between our filtration and the Weiss tower, and therefore no analogue for our main conjecture.

One final remark. Conjecture 12.5 implies that the homotopy spectral sequence associated with the symmetric power filtration, as well as that associated to our filtration of $b u$, collapse at $E_{2}$. (Of course, in the case of the symmetric power filtration, the collapsing at $E_{2}$ is a theorem.) Taken together with Conjecture 12.1, this would imply that an analogous collapsing result holds for the homotopy spectral sequences associated with the Taylor towers (11.1) and (11.2). In the case of the Goodwillie tower, this was first pointed out by N. Kuhn in [10]. 


\section{REFERENCES}

[1] G. Arone, The Weiss derivatives of $B \mathrm{O}(-)$ and $B \mathrm{U}(-)$. Topology 41 (2002), no. 3, 451-481.

[2] G. Arone and W. Dwyer, Partition complexes, Tits buildings and symmetric products. Proc. London Math. Soc. 82 (2001), 229-256.

[3] G. Arone and M. Mahowald, The Goodwillie tower of the identity functor and the unstable periodic homotopy of spheres. Invent. Math. 135 (1999) 743-788.

[4] J. Becker, Characteristic classes and $K$-theory. Lecture Notes in Math., Vol. 428, 132-143, Springer, Berlin, 1974.

[5] W. Dwyer, Homology decompositions for classifying spaces of finite groups. Topology 36 (1997) 783-804.

[6] T. Goodwillie, Calculus. III. Taylor series. Geom. Topol. 7 (2003), 645-711.

[7] N. Kuhn, A Kahn-Priddy sequence and a conjecture of G. W. Whitehead. Math. Proc. Cambridge Philos. Soc. 92 (1982), no. 3, 467-483.

[8] N. Kuhn, Spacelike resolutions of spectra. Proceedings of the Northwestern Homotopy Theory Conference (Evanston, Ill., 1982), 153-165, Contemp. Math., 19, Amer. Math. Soc., Providence, RI, 1983.

[9] N. Kuhn and S. Priddy, The transfer and Whitehead's conjecture. Math. Proc. Cambridge Philos. Soc. 98 (1985), no. 3, 459-480.

[10] N. Kuhn, Goodwillie towers and chromatic homotopy: an overview. Preprint, 2004.

[11] K. Lesh, Infinite loop spaces from group theory. Math. Z. 225 (1997) 467-483.

[12] K. Lesh, A filtration of spectra arising from families of subgroups of symmetric groups. Trans. Amer. Math. Soc. 352 (2000), no. 7, 3211-3237.

[13] J. May, The Wirthmüller isomorphism revisited. Theory Appl. Categ. 11 (2003), no. 5, $132-142$.

[14] S. Mitchell and S. Priddy, Stable splittings derived from the Steinberg module. Topology 22 (1983), no. 3, 285-298.

[15] R. Oliver, $p$-stubborn subgroups of classical compact Lie groups. J. Pure Appl. Algebra 92 (1994), no. 1, 55-78.

[16] D. Quillen, Higher algebraic $K$-theory, I. Algebraic $K$-theory I (Battelle Institute Conference 1972), Lecture Notes in Mathematics 341, Springer-Verlag, 1973.

[17] J. Rognes, A spectrum level rank filtration in algebraic $K$-theory. Topology 31 (1992), no. 4, 813-845.

[18] G. Segal, The stable homotopy of complex projective space. Quart. J. Math. Oxford Ser. (2) 24 (1973), 1-5.

[19] G. Segal, Categories and cohomology theories. Topology 13 (1974) 293-312.

[20] $R$. Thomason, First quadrant spectral sequences in algebraic K-theory via homotopy colimits. Communications in Algebra 10 (1982) 1589-1668

[21] M. Weiss, Orthogonal calculus. Trans. Amer. Math. Soc. 347 (1995), no. 10, 3743-3796.

[22] A. Zolotykh, Classification of projective representations of finite Abelian groups. Moscow University Mathematics Bulletin 57 (2002), 1-8.

Kerchof Hall, U. of Virginia, P.O. Box 400137, Charlottesville VA 22904 USA

E-mail address: zga2m@virginia.edu

Department of Mathematics, Union College, Schenectady NY 12308 USA

E-mail address: leshk@union.edu 